\title{
Action-angle Maps and Scattering Theory for Some Finite-dimensional Integrable Systems III. Sutherland Type Systems and their Duals*
}

By

\author{
Simon RuiJsenaARS **
}

\begin{abstract}
We present an explicit construction of an action-angle map for the nonrelativistic $N$-particle Sutherland system and for two different generalizations thereof, one of which may be viewed as a relativistic version. We use the map to obtain detailed information concerning dynamical issues such as oscillation periods and equilibria, and to obtain simple formulas for partition functions. The nonrelativistic and relativistic Sutherland systems give rise to dual integrable systems with a solitonic long-time asymptotics that is explicitly described. We show that the second generalization is self-dual, and that its reduced phase space can be densely embedded in $\mathbf{P}^{N-1}$ with its standard Kähler form, yielding commuting global flows. In a certain limit the reduced action-angle map converges to the quotient of Fourier transformation on $\mathbf{C}^{N}$ under the standard projection $\mathbf{C}^{N} \backslash\{0\} \rightarrow \mathbf{P}^{N-1}$.
\end{abstract}

\section{Contents}

1 Introduction and Summary

1.1 Introduction

1.2 Summary of Sections 2.1, 2.2, 3.1, 3.2 and 4.1, 4.2

1.3 Summary of Sections 2.3, 3.3, 4.3 and 4.4

1.4 Outline of Chapter 5

2 The Action-angle Transform : Algebraic Aspects

2.1 The Case $\mathrm{III}_{\mathrm{nr}}$

2.2 The Case $\mathrm{II}_{\text {rel }}$

2.3 The Case III $_{\mathrm{b}}$

Communicated by T. Miwa, May 11, 1994.

1991 Mathematics Subject Classifications : 58F07.

* Work partly supported by the Netherlands Organisation for the Advancement of Research (NWO).

** Centre for Mathematics and Computer Science, P. O. Box 4079, 1009 AB Amsterdam, The Netherlands. 
3 The Action-angle Transform : Analytic and Geometric Aspects

3.1 The Case $\mathrm{III}_{\mathrm{nr}}$

3.2 The Case $\mathrm{II}_{\text {rel }}$

3.3 The Case III $_{\mathrm{b}}$

4 The Harmonic Oscillator Transform

4.1 The Case III $_{\mathrm{nr}}$

4.2 The Case $\mathrm{II}_{\text {rel }}$

4.3 The Case III $_{\mathrm{b}}$

4.4 The III $_{\mathrm{b}}$ Map $\phi$

5 Dynamics and Scattering

5.1 The Cases III $_{\mathrm{nr}}$ and $\mathrm{III}_{\mathrm{nr}}$

5.2 The Cases III $_{\text {rel }}$ and $\hat{\mathrm{III}}_{\text {rel }}$

5.3 The Case III $_{\mathrm{b}}$

Appendix A. Some Algebraic Lemmas

References

\section{Introduction and Summary}

\subsection{Introduction}

In two previous papers $[1,2]$ (henceforth referred to as $I$, II) we have studied Calogero-Moser type $N$-particle dynamics whose long-time asymptotics can be encoded in a soliton-like scattering transformation. In this paper our starting point consists of the closely related Sutherland dynamics

$$
H=\frac{1}{2} \sum_{j=1}^{N} p_{j}^{2}+\frac{1}{4} g^{2}|\mu|^{2} \sum_{1 \leq j<k \leq N} \frac{1}{\sin ^{2} \frac{|\mu|}{2}\left(x_{j}-x_{k}\right)} \quad\left(\mathrm{III}_{\mathrm{nr}}\right)
$$

and two integrable generalizations thereof, viz.,

$$
\begin{aligned}
& H=\sum_{j=1}^{N} \operatorname{ch}\left(\beta p_{j}\right) V_{j}(x), \quad \beta \in(0, \infty) \quad\left(\text { III }_{\text {rel }}\right) \\
& V_{j} \equiv \prod_{k \neq j}\left(1+\frac{\operatorname{sh}^{2} z}{\sin ^{2} \frac{|\mu|}{2}\left(x_{j}-x_{k}\right)}\right)^{1 / 2} \\
& H=\sum_{j=1}^{N} \cos \left(|\beta| p_{j}\right) V_{j}^{b}(x), \quad \beta \in i(0, \infty) \quad\left(\text { III }_{\mathrm{b}}\right) \\
& V_{j}^{b} \equiv \prod_{k \neq j}\left(1-\frac{\sin ^{2} \tau}{\sin ^{2} \frac{|\mu|}{2}\left(x_{j}-x_{k}\right)}\right)^{1 / 2}, \quad \tau \in(0, \pi / N)
\end{aligned}
$$


We may and will view these dynamics as the simplest non-trivial representants of three classes of commuting dynamics, each of which has an associated class of 'dual' dynamics. The former dynamics yield oscillatory motion, but the dynamics dual to (1.1) and (1.2) (which are exemplified by (1.106)-(1.108)) have a solitonic long-time asymptotics. The integrable systems associated with (1.4) will be shown to be self-dual.

Unless explicitly stated otherwise, we take

$$
\mu \in i(0, \infty), \quad g<0
$$

Setting

$$
z \equiv i \beta \mu g / 2, \quad \tau \equiv \beta \mu g / 2
$$

this convention entails $z>0$ for the case $\mathrm{III}_{\text {rel }}$ (where $\beta>0$ ) and $\tau>0$ for the case $\mathrm{III}_{\mathrm{b}}$ (where $\left.-i \beta>0\right)$. We suppress dependence on the parameters whenever this causes no ambiguities. (The sign of $g$ is fixed to ease the definition of certain matrices; the various maps and dynamics occurring below are even in $\mathrm{g}$, cf. also I Proposition 5.5.)

To a large extent this paper is self-contained. However, we do need the spectral asymptotics and canonicity results obtained in I. For more context and background material concerning the integrable systems at hand, we refer to [3-6]. In particular, in our survey [6] we discuss both the classical and the quantum versions of the above-mentioned systems, their elliptic generalizations and Toda type limits, and their relations to infinite-dimensional integrable systems. Also, in [4] the terminology 'nonrelativistic' (nr) vs. 'relativistic' (rel) is explained, cf. also $[5,7]$. (To model solid-state phenomena it may be more natural to replace the speed of light by the speed of sound, e.g.) The suffix b in (1.4) stands for 'bounded'.

In contrast to the dynamics handled in I and II (whose interpretation is unambiguous), there exist three different interpretations of the Hamiltonians (1.1) and (1.2), whereas (1.4) admits even more than three versions. To explain this, we introduce the Weyl alcoves

$$
\begin{gathered}
\mathbf{W}_{n} \equiv\left\{\delta \in \mathbb{R}^{n}\left|\delta_{1}, \ldots, \delta_{n}>0, \quad\right| \mu \mid \sum_{j=1}^{n} \delta_{j}<\pi\right\}, \quad n \equiv N-1 \\
W_{N} \equiv\left\{q \in \mathbb{R}^{N} \mid\left(q_{1}-q_{2}, q_{2}-q_{3}, \ldots, q_{n}-q_{N}\right) / 2 \in \mathbf{W}_{n}\right\}
\end{gathered}
$$

Clearly, $W_{N}$ is an open convex set, so we obtain a symplectic manifold $\langle\tilde{\Omega}, \tilde{\omega}\rangle$ by setting

$$
\begin{gathered}
\tilde{\Omega} \equiv\left\{(q, \theta) \in \mathbb{R}^{2 N} \mid q \in W_{N}\right\} \\
\tilde{\omega} \equiv \sum_{j=1}^{N} d q_{j} \wedge d \theta_{j}
\end{gathered}
$$


Replacing $x, p$ by $q, \theta$ at the rhs of (1.1)-(1.3), we obtain smooth positive functions on $\tilde{\Omega}$. The Hamiltonians defined in this way will be denoted by $\tilde{H}$.

Next, consider the $\mathbb{Z}$-action on $\tilde{\Omega}$ whose generator reads

$\boldsymbol{G}:\left(q_{1}, \ldots, q_{N}, \theta_{1}, \ldots, \theta_{N}\right) \longmapsto\left(q_{N}+2 \pi /|\mu|, q_{1}, \ldots, q_{N-1}, \theta_{N}, \theta_{1}, \ldots, \theta_{N-1}\right)$

This action is well defined, free, discrete and symplectic, and the functions $\tilde{H}$ are $\mathbf{Z}$-invariant. Thus we may divide out this action to obtain a manifold

$$
\Omega \equiv \tilde{\Omega} / \mathbb{Z}
$$

equipped with a symplectic form $\omega$, and smooth functions $H$ on $\Omega$. We coordinatize $\Omega$ by setting

$$
\Omega \simeq\left\{(x, p) \in \mathbb{R}^{2 N} \mid x \in F_{N}\right\}
$$

where $F_{N}$ is defined by

$$
F_{N} \equiv\left\{x \in \mathbb{R}^{N}|-\pi /| \mu\left|<x_{N}<\cdots<x_{1} \leq \pi /\right| \mu \mid\right\}
$$

This is a natural choice, since $F_{N}$ is a fundamental set for the $\mathbb{Z}$-action restricted to $W_{N}$. To be more specific, given $(q, \theta) \in \tilde{\Omega}$, there exist uniquely determined $x \in F_{N}$, $l \in\{1, \ldots, N\}$ and $m \in \mathbb{Z}$ such that

$$
\begin{gathered}
x_{1}=q_{l}+2 \pi m /|\mu| \\
\vdots \\
x_{N-l+1}=q_{N}+2 \pi m /|\mu| \\
x_{N-l+2}=q_{1}+2 \pi(m-1) /|\mu| \\
\vdots \\
x_{N}=q_{l-1}+2 \pi(m-1) /|\mu|
\end{gathered}
$$

and then $p$ is defined by

$$
\begin{gathered}
p_{1}=\theta_{l} \\
\vdots \\
p_{N-l+1}=\theta_{N} \\
p_{N-l+2}=\theta_{1} \\
\vdots \\
p_{N}=\theta_{l-1}
\end{gathered}
$$


We may and will view (1.1) and (1.2) as the coordinate expressions for the smooth functions $H$ on $\Omega$ we have just defined. Note that on the open dense coordinate patch $\left\{x_{1}<\pi /|\mu|\right\}$ one has

$$
\omega=\sum_{j=1}^{N} d x_{j} \wedge d p_{j}
$$

For later purposes it is expedient to insert an important observation at this point : two vectors $q, \tilde{q} \in W_{N}$ belong to the same orbit under the $\mathbf{Z}$-action if and only if the diagonal matrices $A(q)$ and $A(\tilde{q})$ are related by a permutation, where $A$ is defined by

$$
A(y) \equiv \operatorname{diag}\left(e^{\mu y_{1}}, \ldots, e^{\mu y_{N}}\right)
$$

(Note that the permutation involved is necessarily cyclic.)

Physically speaking, the Hamiltonians $\tilde{H}(q, \theta)$ on the phase space $\tilde{\Omega}$ describe $N$ particles on the line whose distances are bounded below and above due to energy conservation. Hence they can be distinguished by their ordering. The Hamiltonians $H(x, p)$ on $\Omega$ describe particles on a ring, whose angular positions are encoded in the phase factors $\exp \left(\mu x_{j}\right) \in S^{1} \subset \mathbf{C}$. Then the ordering is fixed up to a cyclic permutation. Factoring out the $\mathbf{Z}$-action generated by $\mathbf{G}$ amounts to viewing the particles as indistinguishable.

However, one may also treat the particles as being distinguishable. Then one needs a phase space

$$
\Omega^{\prime} \equiv \tilde{\Omega} / \mathbf{Z}^{\prime}
$$

where $\mathbf{Z}^{\prime}$ denotes the $\mathbf{Z}$-action generated by

$$
G^{N}:(q, \theta) \longmapsto\left(q_{1}+2 \pi /|\mu|, \ldots, q_{N}+2 \pi /|\mu|, \theta\right)
$$

One way to coordinatize $\Omega^{\prime}$ and the quotient form $\omega^{\prime}$ is to take

$$
\Omega^{\prime} \simeq\left\{(y, k) \in \mathbf{R}^{2 N} \mid y \in F_{N}^{\prime}\right\}, \quad \omega^{\prime} \simeq \sum_{j=1}^{N} d y_{j} \wedge d k_{j}
$$

where

$$
F_{N}^{\prime} \equiv\left\{y \in W_{N} \mid \sum_{j=1}^{N} y_{j} \in(-\pi N /|\mu|, \pi N /|\mu|]\right\}
$$

That is, for a given $(q, \theta) \in \tilde{\Omega}$ one has

$$
y_{j}=q_{j}+2 \pi m /|\mu|, \quad k_{j}=\theta_{j}, \quad j=1, \ldots, N
$$


where $m \in \mathbb{Z}$ is uniquely determined. Replacing $x, p$ by $y, k$ in (1.1)-(1.3) yields smooth functions $H^{\prime}(y, k)$ on $\Omega^{\prime}$. Note that one has

$$
\Omega \simeq \Omega^{\prime} / \mathbb{Z}_{N}
$$

where the $\mathbb{Z}_{N}$-action consists in cyclic permutations of $y, k$; this amounts to writing

$$
\mathbb{Z} \simeq \mathbb{Z}_{N} \times \mathbb{Z}^{\prime}
$$

Summarizing, we have

$$
(q, \theta) \in \tilde{\Omega} \stackrel{\pi\left(\mathbf{Z}^{\prime}\right)}{\longrightarrow}(y, k) \in \Omega^{\prime} \stackrel{\pi\left(\mathbf{Z}_{x}\right)}{\longrightarrow}(x, p) \in \Omega
$$

(Here and below, $\pi(\bullet)$ denotes regular covering projections.) As far as dynamics is concerned, our emphasis will be on understanding a class of commuting Hamiltonian flows on $\tilde{\Omega}$ that contains in particular the flow generated by $\tilde{H}$. All of these flows admit quotient flows on $\Omega^{\prime}$ and $\Omega$, whose relevant features can be read off from the covering sequence (1.27).

Just as in I and II, we shall arrive at a detailed understanding of the commuting flows via an explicit picture of the action-angle map and its 'harmonic oscillator' extension. These maps are most easily constructed at the left side of the sequence (1.27), since $\tilde{\Omega}$ is convex and hence topologically trivial. Moreover, a linear coordinate change turns $\tilde{\Omega}$ into a product of $\mathbb{R}^{2}$ and an open convex subset $M$ of $\mathbb{R}^{2 n}$, encoding the center of mass motion and reduced (center of mass frame) motion, resp., for the Sutherland dynamics. Correspondingly, the action-angle map can be factorized. Since this change of coordinates

$$
\mathscr{C}: \tilde{\Omega} \stackrel{\longrightarrow}{\longrightarrow} \mathbb{R}^{2} \times M, \quad(q, \theta) \longmapsto\left(\delta_{s}, \gamma_{s} ; \delta, \gamma\right)
$$

plays a key role in the sequel, we detail it now :

$$
\begin{aligned}
& \delta_{s}=\left(q_{1}+\cdots+q_{N}\right) / N \quad q_{1}=\delta_{s}+2\left[n \delta_{1}+(n-1) \delta_{2}+\cdots+\delta_{n}\right] / N \\
& \delta_{1}=\left(q_{1}-q_{2}\right) / 2 \quad q_{2}=\delta_{s}+2\left[-\delta_{1}+(n-1) \delta_{2}+\cdots+\delta_{n}\right] / N \\
& \text { : } \\
& \delta_{n}=\left(q_{n}-q_{N}\right) / 2 \\
& \Longleftrightarrow \quad \text { : } \\
& q_{N}=\delta_{s}+2\left[-\delta_{1}-2 \delta_{2}-\cdots-n \delta_{n}\right] / \mathbb{N} \\
& \gamma_{s}=\left(\theta_{1}+\cdots+\theta_{N}\right) / N \\
& \gamma_{1}=\theta_{1}-\left(\theta_{1}+\cdots+\theta_{N}\right) / N \\
& \theta_{1}=\gamma_{s}+\gamma_{1} \\
& \vdots \\
& \theta_{2}=\gamma_{s}+\gamma_{2}-\gamma_{1} \\
& \gamma_{n}=\theta_{1}+\cdots+\theta_{n}-n\left(\theta_{1}+\cdots+\theta_{N}\right) / N \\
& \Longleftrightarrow \begin{array}{c}
\theta_{2}=\gamma_{s}+\gamma_{2}-\gamma_{1} \\
\vdots \\
\theta_{N}=\gamma_{s}-\gamma_{n}
\end{array}
\end{aligned}
$$

(Recall $n \equiv N-1$.$) Clearly, this entails$ 


$$
M=\mathbf{W}_{n} \times \mathbf{R}^{n}
$$

Also, the new coordinates are not quite canonical ; setting

$$
\begin{gathered}
\omega_{s} \equiv N d \delta_{s} \wedge d \gamma_{s} \\
\omega(M) \equiv 2 \sum_{j=1}^{n} d \delta_{j} \wedge d \gamma_{j}
\end{gathered}
$$

one easily checks that

$$
\mathscr{C}^{*}\left(\omega_{s}+\omega(\boldsymbol{M})\right)=\tilde{\omega}
$$

(Of course, the scale factors 2 and $N$ are a matter of convention. Our choice ensures absence of similar factors in many later formulas.)

We now turn to the $\mathrm{III}_{\mathrm{b}}$ systems. Here, we need the open convex sets

$$
\begin{gathered}
\mathbf{W}_{n}^{b} \equiv\left\{\delta \in \mathbf{R}^{n}|| \mu\left|\delta_{1}, \ldots,\right| \mu\left|\delta_{n}>\tau>0, \quad\right| \mu \mid \sum_{j=1}^{n} \delta_{j}<\pi-\tau\right\} \\
W_{N}^{b} \equiv\left\{q \in \mathbf{R}_{N} \mid\left(q_{1}-q_{2}, \ldots, q_{n}-q_{N}\right) / 2 \in \mathbf{W}_{n}^{b}\right\}
\end{gathered}
$$

Note that the restriction $\tau<\pi / N$ in (1.5) is equivalent to $\mathrm{w}_{n}^{b}$ being non-empty. Now we put

$$
\tilde{\Omega} \equiv\left\{(q, \theta) \in \mathbf{R}^{2 N} \mid q \in W_{N}^{b}\right\}
$$

and equip $\tilde{\Omega}$ with the symplectic form $\tilde{\omega}$, cf. (1.11). As before, replacing $x, p$ by $q$, $\theta$ at the rhs of (1.4), (1.5) yields a smooth real-valued function $\tilde{H}$ on $\tilde{\Omega}$. (The restriction on $q$ guarantees that all radicands in (1.5) are positive.)

Next, we define a $\mathbb{Z}^{N}$-action on $\tilde{\Omega}$ by setting

$$
\theta \longmapsto \theta+2 \pi k /|\beta|, \quad k \in \mathbf{Z}^{N}
$$

and a $\mathbf{Z}$-action via the generator $G$, cf. (1.12). Combining these, we obtain a free action of a semi-direct product of $\mathbf{Z}$ and $\mathbf{Z}^{N}$ (the action of $\mathbf{Z}$ on $\mathbf{Z}^{N}$ being generated by $\left.\left(k_{1}, \ldots, k_{N}\right) \longmapsto\left(k_{N}, k_{1}, \ldots, k_{N-1}\right)\right)$. This action is symplectic and leaves $\tilde{H}$ invariant, so $\widetilde{H}$ descends to a smooth funtion $H$ on the quotient symplectic manifold $\langle\Omega, \omega\rangle$, where

$$
\Omega \equiv \tilde{\Omega} /\left(\mathbf{Z} \ltimes \mathbf{Z}^{N}\right)
$$

Introducing 


$$
F_{N}^{b} \equiv W_{N}^{b} \cap F_{N}
$$

an obvious coordinatization of $\Omega$ reads

$$
\Omega \simeq\left\{(x, p) \in \mathbb{R}^{2 N} \mid x \in F_{N}^{b}, p \in(-\pi /|\beta|, \pi /|\beta|]^{N}\right\}
$$

Here, $x$ is defined via (1.16), whereas the definition (1.17) of $p$ should now be read $\bmod 2 \pi /|\beta|$.

Clearly, upon quotienting out part of the $\mathbb{Z} \ltimes \mathbb{Z}^{N}$-action one can obtain various phase spaces and Hamiltonians interpolating between $\langle\tilde{\Omega}, \tilde{\omega}\rangle, \tilde{H}$ and $\langle\Omega, \omega\rangle, \mathbb{H}$, each with its own physical interpretation. We single out one of these, because its action-angle map is most easily constructed.

To this purpose we introduce the subgroup

$$
E_{N} \equiv\left\{k \in \mathbb{Z}^{N} \mid \sum_{j=1}^{N} k_{j}=0\right\}
$$

Changing coordinates on $\tilde{\Omega}$ via (1.29) and (1.30), the action of $E_{N}$ leaves $\left(\delta_{s}, \gamma_{s}\right)$ $\in \mathbb{R}^{2}$ and $\delta \in W_{n}^{b}$ fixed, whereas $\gamma_{1}, \ldots, \gamma_{n}$ change by multiples of $2 \pi /|\beta|$. Then the quotient manifold

$$
\Omega^{c} \equiv \tilde{\Omega} / E_{N}
$$

may and will be viewed as

$$
\Omega^{c}=\mathbb{R}^{2} \times M^{0}, \quad M^{0} \equiv \mathrm{W}_{n}^{b} \times \mathbb{T}^{n}
$$

Here and from now on $\mathbb{T}^{l}$ denotes the torus

$$
\mathbb{T}^{l} \equiv\left\{\nu \in \mathbb{C}^{l}|| \nu_{1}|=\cdots=| \nu_{l} \mid=1\right\}
$$

The obvious coordinates on $\Omega^{c}$ are $\delta_{s}, \gamma_{s}, \delta$ and $\gamma$, with $\gamma$ now varying over $(-\pi /|\beta|, \pi /|\beta|]^{n}$. The corresponding quotient form and Hamiltonian will be denoted by $\omega^{c}$ and $H^{c}$, resp. Thus we have

$$
\omega^{c}=\omega_{s}+\omega\left(M^{0}\right)
$$

where $\omega_{s}$ is given by (1.32) and $\omega\left(M^{0}\right)$ by (the obvious reinterpretation of) the rhs of (1.33).

Having prepared the arena, the battle can begin. Lest the logistics go haywire, we suggest that the reader skip the following two sections at first reading. These sections contain a rather detailed summary of Chapters 2-4 and might be referred back to as needed. (This is perhaps the best policy as regards all of Chapters 2-4.) 
Quite a few auxiliary objects and a lot of notation must be introduced to define and study the various maps and spaces that are relevant for the systems at hand, and some of this is already necessary to even sketch their constructions. Thus Sections 1.2 and 1.3 should serve both as a database and as a flow chart, uncluttered by the many technicalities arising in Chapters 2-4 and Appendix A. In Section 1.4 we summarize Chapter 5 in a more descriptive fashion.

We close this introductory section with three more remarks. First, we would like to point out that many of the $\mathbb{I I}_{\text {rel }}$ and $\mathrm{III}_{\mathrm{b}}$ objects reduce to their $\mathrm{II}_{\mathrm{nr}}$ counterparts when one fixes $g$ and $\mu$, renormalizes suitably, and takes $\beta$ to 0 . (Similarly, taking $\mu$ to 0 in the $\mathrm{III}_{\text {rel }}$ systems and their duals, one obtains the $\mathrm{I}_{\text {rel }}$ and $\Pi_{n \mathrm{r}}$ systems, resp., studied in I.) But just as in $\mathrm{I}$ and $I$, we do not have sufficient control over this limit to rigorously obtain all of the $\mathrm{II}_{\mathrm{nr}}$ results as corollaries of the $\mathrm{III}_{\text {rel }}$ and/or $\mathrm{III}_{\mathrm{b}}$ results. Therefore, we handle the $\mathrm{II}_{\mathrm{nr}}$ systems separately, choosing however notation and arguments that admit partial generalization to the $\mathrm{II}_{\text {rel }}$ and III $\mathrm{b}$ systems.

Secondly, a part of this paper can be reformulated in terms of notions from the area of Lie groups, Lie algebras and symmetric spaces ((affine) Weyl groups, root and weight lattices, totally geodesic submanifolds, to name a few) ; the afficionado will have little trouble doing so. (Cf. also [3] for this viewpoint.)

Thirdly, we remark that the above-mentioned dual dynamics emerge as a corollary of the constructions in Chapters 2-4. As such, we have deferred their definition to the beginning of Section 1.4. At this point, the dual dynamics (1.106), (1.107) appears to be very far removed from the Sutherland dynamics (1.1). As will be seen below, however, the inverse of the harmonic oscillator map for the latter dynamics serves as the action-angle map for the former-a quite unexpected and most remarkable bonus. Similarly, the self-duality of the dynamics (1.4), (1.5) amounts to the corresponding harmonic oscillator map being (in essence) involutive. (Cf. also our previous paper I, where the notion of 'dual system' is more readily understood.)

\subsection{Summary of Sections 2.1, 2.2, 3.1, 3.2 and 4.1, 4.2}

We begin by discussing the $\mathrm{III}_{\mathrm{nr}}$ and $\mathrm{II}_{\text {rel }}$ systems, which can be handled in much the same way. First of all, the commuting Hamiltonians can be obtained from an $N \times N$ matrix-valued function $L$ on $\tilde{\Omega}$, which is self-adjoint in both cases. This Lax matrix is defined by (2.1) and (2.51), resp. Just as in I and II, its spectral properties are an essential ingredient for the explicit construction of the action-angle map. Once again, the starting point for obtaining detailed spectral information is the commutation relation between $L(q, \theta)$ and the matrix $A(q)$ given by (1.19). (This relation was first used for the $\mathrm{II}_{\mathrm{nr}}$ systems in $[8,9]$, cf. also [10].) 
Combining the commutation relation with algebraic information assembled in Appendix A, we show in Section 2.1 that $L$ has eigenvalues $\lambda_{1}, \ldots, \lambda_{N}$ satisfying $\left|\lambda_{i}-\lambda_{j}\right| \geq|\mu \mathrm{g}|, i \neq j$; in Section 2.2 we obtain positive eigenvalues obeying $\mid \ln \lambda_{i}$ $-\ln \lambda_{j} \mid \geq 2 z, i \neq j$. The subset of $\widetilde{\Omega}$ where all inequalities are strict is denoted by $\tilde{\Omega}_{r}$. (Here, $r$ stands for 'regular'. On the boundary set $\tilde{\Omega}_{b} \equiv \tilde{\Omega} \backslash \tilde{\Omega}_{r}$ the dimension of the vector space spanned by the gradients of the commuting Hamiltonians is smaller than $N$.)

It is convenient to view $\tilde{\Omega}$ as $\mathbf{R}^{2} \times M, M=\mathrm{W}_{n} \times \mathbf{R}^{n}$, via the above coordinate change $\mathscr{C}$, cf. (1.28) $-(1.30)$. Then the spectral requirement amounts to a restriction on $M$ :

$$
\tilde{\Omega}_{r} \simeq \mathbf{R}^{2} \times M_{r}
$$

Fixing $P \in \tilde{\Omega}_{r}$, there exists a unitary matrix $U(P)$ such that

$$
\left(U^{*} L U\right)(P)= \begin{cases}\operatorname{diag}\left(\hat{\theta}_{1}, \ldots, \hat{\theta}_{N}\right) & \left(\mathrm{III}_{\mathrm{nr}}\right) \\ \operatorname{diag}\left(e^{\beta \hat{\theta}_{1}}, \ldots, e^{\beta \hat{\theta}_{N}}\right) & \left(\mathrm{III}_{\mathrm{rel}}\right)\end{cases}
$$

where $\hat{\theta}$ varies over the action set

$$
A_{N} \equiv\left\{\hat{\theta} \in \mathbf{R}^{N} \mid \hat{\theta}_{j}-\hat{\theta}_{j+1}>d, \quad j=1, \ldots, n\right\}, \quad d \equiv|\mu g|
$$

Introducing

$$
\begin{aligned}
& \hat{\delta}_{s} \equiv\left(\hat{\theta}_{1}+\cdots+\hat{\theta}_{N}\right) / N \\
& \hat{\delta}_{j} \equiv\left(\hat{\theta}_{j}-\hat{\theta}_{j+1}\right) / 2, \quad j=1, \ldots, n
\end{aligned}
$$

it follows that $\hat{\delta}_{s}$ varies over $\mathbf{R}$ and $\hat{\delta}$ over

$$
a_{n} \equiv\left\{\hat{\delta} \in \mathbf{R}^{n} \mid \hat{\delta}_{1}, \ldots, \hat{\delta}_{n}>d / 2\right\}
$$

In both cases we obtain

$$
\hat{\delta}_{s}=\gamma_{s}
$$

A suitable fixing of the gauge freedom left in $U$ now gives rise to $n$ phase factors that are written $\exp \left(\mu \hat{\gamma}_{j}\right), \hat{\gamma}_{j} \in(-\pi /|\mu|, \pi /|\mu|], j=1, \ldots, n$. Setting

$$
\hat{\gamma}_{s}=\delta_{s}
$$

we then obtain the (action-angle) map 


$$
\tilde{\Phi}: \mathbf{R}^{2} \times M_{r} \longrightarrow \hat{\Omega}^{c},\left(\delta_{s}, \gamma_{s} ; \delta, \gamma\right) \longmapsto\left(\hat{\delta}_{s}, \hat{\gamma}_{s} ; \hat{\delta}, \hat{\gamma}\right)
$$

where

$$
\hat{\Omega}^{c} \equiv \mathbf{R}^{2} \times \hat{M}^{0}, \quad \hat{M}^{0} \equiv \mathrm{a}_{n} \times \mathbf{T}^{n}
$$

Furthermore, the map can be factorized as

$$
\tilde{\Phi}=P_{2} \times \phi_{r}
$$

Here and below, $P_{2 k}$ denotes the flip map on $\mathbf{R}^{2 k}$ :

$$
P_{2 k}(x, y) \equiv(y, x), \quad x, y \in \mathbf{R}^{k}
$$

The detailed construction of $\tilde{\Phi}$ can be found in Sections 2.1 and 2.2 for the cases III $_{\mathrm{nr}}$ and $\mathrm{III}_{\text {rel }}$, resp. It is proved there that $\tilde{\Phi}$ is a bijection onto $\hat{\Omega}^{c}$. Moreover, the $\hat{\mathbf{Z}}$-action on $\hat{\Omega}^{c}$ corresponding to the $\mathbf{Z}$-action on $\tilde{\Omega}$ is determined, cf. Lemmas 2.1, 2.2. We shall write the inverse of $\tilde{\Phi}$ as

$$
\tilde{\mathscr{E}}=P_{2} \times b^{0}, \quad b^{0} \equiv \phi_{r}^{-1}
$$

(Thus far, our notation may appear somewhat bizarre. However, we are anticipating an extension to all of $\tilde{\Omega}$, as well as the self-duality of the case $\mathbb{I I I}_{\mathrm{b}}$. Once the whole picture has been sketched, we hope the patient reader will agree that our notation is appropriate.)

The principal result of Sections 3.1 and 3.2 is that the map $\tilde{\Phi}$ is a real-analytic (henceforth $C^{\omega}$ ) symplectomorphism from $\left\langle\tilde{\Omega}_{r}, \tilde{\omega}\right\rangle$ onto $\left\langle\mathbf{R}^{2} \times \hat{M}^{0}, \hat{\omega}^{c}\right\rangle$, where

$$
\begin{gathered}
\hat{\omega}^{c} \equiv N d \hat{\gamma}_{s} \wedge d \hat{\delta}_{s}+\omega\left(\hat{M}^{0}\right) \\
\omega\left(\hat{M}^{0}\right) \equiv 2 \sum_{j=1}^{n} d \hat{\gamma}_{j} \wedge d \hat{\delta}_{j}
\end{gathered}
$$

This is proved by exploiting the canonicity results obtained in I. In brief, a suitably chosen analytic continuation yields a branch

$$
\boldsymbol{B}: \tilde{\hat{\Omega}} \equiv \mathbf{R}^{N} \times A_{N} \longrightarrow \tilde{\Omega}_{r}, \quad(\hat{q}, \hat{\theta}) \longmapsto(q, \theta)
$$

of the multi-valued holomorphic function $R$ from II Sections 3A and 3B. The continuation preserves canonicity, so that

$$
B^{*} \tilde{\omega}=\tilde{\hat{\omega}}
$$


where

$$
\tilde{\hat{\omega}} \equiv \sum_{j=1}^{N} d \hat{q}_{j} \wedge d \hat{\theta}_{j}
$$

Then we define a coordinate change $\hat{\mathscr{C}}$ by $(1.50)$ and by

$$
\begin{aligned}
& \hat{\gamma}_{s} \equiv\left(\hat{q}_{1}+\cdots+\hat{q}_{N}\right) / N \\
& \hat{\gamma}_{j} \equiv \hat{q}_{1}+\cdots+\hat{q}_{j}-j\left(\hat{q}_{1}+\cdots+\hat{q}_{N}\right) / N, j=1, \ldots, n
\end{aligned}
$$

and a $\hat{\mathbf{Z}}^{N}$-action by

$$
\hat{q} \longmapsto \hat{q}+2 \pi k /|\mu|, \quad k \in \hat{\mathbb{Z}}^{N}
$$

Upon quotienting out the $\hat{E}_{N}$-subgroup (given by (1.42)), the following commutative diagram arises :

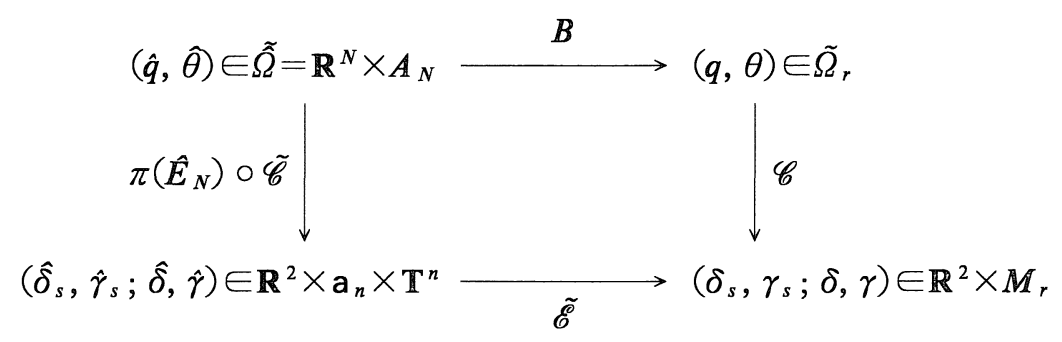

Since the quotient form $\tilde{\hat{\omega}} / E_{N}$ equals $\hat{\omega}^{c}$, the salient properties of $\tilde{\mathscr{E}}$ can now be read off : in addition to being bijective (as already shown in Chapter 2), $\tilde{\mathscr{E}}$ is $C^{\omega}$ and symplectic.

The situation on $\Omega_{r}$ can then be understood by dividing out the $\mathbb{Z}$-action on $\tilde{\Omega}_{r}$ and the corresponding $\hat{\mathbb{Z}}$-action on $\hat{\Omega}^{c}$. This yields a commutative diagram

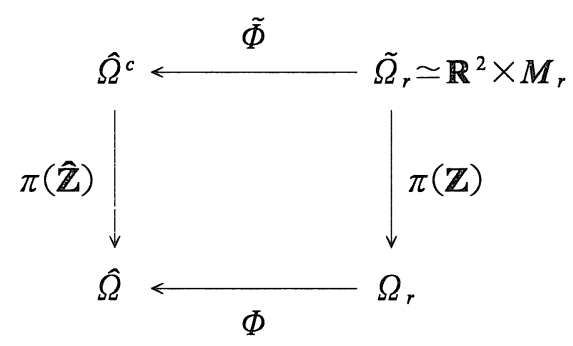

Here, $\hat{\Omega}$ equipped with the quotient form $\hat{\omega}$ is the action-angle phase space corresponding to the starting point $\langle\Omega, \omega\rangle, H$. Explicitly, we may take 


$$
\begin{gathered}
\hat{\Omega}=\mathbf{T}^{N} \times A_{N} \simeq\left\{(\hat{x}, \hat{p}) \in \mathbf{R}^{2 N} \mid \hat{x} \in(-\pi /|\mu|, \pi /|\mu|]^{N}, \hat{p} \in A_{N}\right\} \\
\hat{\omega}=\sum_{j=1}^{N} d \hat{x}_{j} \wedge d \hat{p}_{j}
\end{gathered}
$$

where the coordinates are related to those on $\tilde{\hat{\Omega}}$ by

$$
\hat{p}_{j}=\hat{\theta}_{j}, \quad \hat{x}_{j}=\hat{q}_{j} \quad(\bmod 2 \pi /|\mu|), \quad j=1, \ldots, N
$$

In Sections 4.1 and 4.2 we show that the $C^{\omega}$ symplectomorphism $\phi_{r}:\left\langle M_{r}\right.$, $\left.\omega\left(M_{r}\right)\right\rangle \rightarrow\left\langle\hat{M}^{0}, \omega\left(\hat{M}^{0}\right)\right\rangle$ admits an extension to a $C^{\omega}$ symplectomorphism

$$
\phi:\langle\boldsymbol{M}, \omega(\boldsymbol{M})\rangle \longrightarrow\langle\hat{\boldsymbol{M}}, \omega(\hat{\boldsymbol{M}})\rangle, \quad(\delta, \gamma) \longmapsto(u, v)
$$

with inverse $b$ extending $b^{0}$. Here, one has

$$
\hat{M} \equiv \mathbf{R}^{2 n}, \quad \omega(\hat{M}) \equiv \sum_{j=1}^{n} d u_{j} \wedge d v_{j}
$$

and $\hat{M}^{0}$ is embedded in $\hat{M}$ as the open dense full measure submanifold where $\left(u_{J}\right.$, $\left.v_{j}\right) \neq(0,0), j=1, \ldots, n$. Specifically, we have on $\hat{M}^{0}$

$$
\begin{array}{ccc}
u_{j} \equiv-2\left(\hat{\delta}_{j} /|\mu|-|g| / 2\right)^{1 / 2} \cos |\mu| \hat{\gamma}_{j} & \begin{array}{c}
\hat{\gamma}_{j}=-\operatorname{arctg}\left(v_{j} / u_{j}\right) /|\mu| \\
v_{j} \equiv 2\left(\hat{\delta}_{j} /|\mu|-|g| / 2\right)^{1 / 2} \sin |\mu| \hat{\gamma}_{j}
\end{array} & j=1, \ldots, n \\
& \hat{\delta}_{j}=|\mu|\left(u_{j}^{2}+v_{j}^{2}+2|g|\right) / 4
\end{array}
$$

so $\omega(\hat{M})$ in $(1.72)$ extends $\omega\left(\hat{M}^{0}\right)$ in (1.60). From this result one easily understands the state of affairs on $\tilde{\Omega}$, and a corresponding 'harmonic oscillator' picture for $\Omega$ can then be read off from the following commutative diagram :

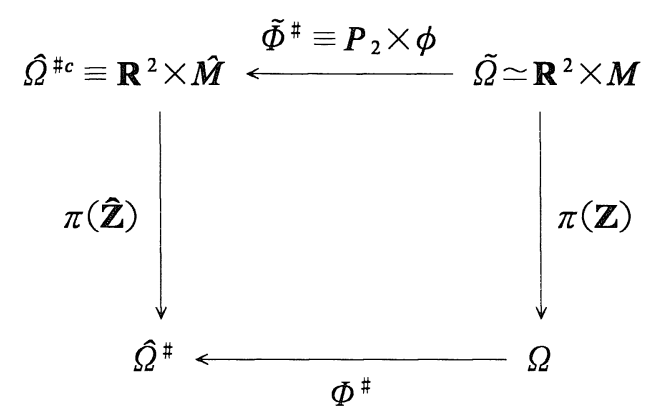




\subsection{Summary of Sections 2.3, 3.3, 4.3 and 4.4}

We proceed by sketching the corresponding results for the case $\mathrm{III}_{\mathrm{b}}$. Here our starting point is the manifold $\Omega^{c}$, cf. (1.43). In contrast to the previous cases, where the Lax matrix is self-adjoint, $L$ is now unitary, $\mathrm{cf}$. (2.82). Once more, $L$ has simple spectrum on $\Omega^{c}$; moreover, the minimal eigenvalue distance (in arclength along $S^{1}$ ) is equal to $2 \tau$. As before, we first restrict attention to the open dense full measure submanifold

$$
\Omega_{r}^{c}=\mathbf{R}^{2} \times M_{r}^{0}
$$

where all distances are larger than $2 \tau$. Fixing $P \in \Omega_{r}^{c}$, there exists a unitary $U(P)$ such that

$$
\left(U^{*} L U\right)(P)=\operatorname{diag}\left(e^{\beta \hat{\theta}_{1}}, \ldots, e^{\beta \hat{\theta}_{N}}\right) \quad\left(\mathbb{I I I}_{\mathrm{b}}\right)
$$

Here, $\hat{\theta}$ is uniquely determined by requiring that it satisfy

$$
\sum_{j=1}^{N} \hat{\theta}_{j}=N \gamma_{s}
$$

and belong to

$$
A_{N}^{b} \equiv\left\{y \in \mathbb{R}^{N} \mid\left(y_{1}-y_{2}, \ldots, y_{n}-y_{N}\right) / 2 \in \mathbf{a}_{n}^{b}\right\}
$$

where

$$
\mathrm{a}_{n}^{b} \equiv\left\{\xi \in \mathbf{R}^{n}|| \beta\left|\xi_{1}, \ldots,\right| \beta\left|\xi_{n}>\tau,\right| \beta \mid \sum_{j=1}^{n} \xi_{j}<\pi-\tau\right\}
$$

Introducing $\hat{\delta}_{s}$ and $\hat{\delta}_{1}, \ldots, \hat{\delta}_{n}$ by (1.50), it then follows from (1.77) that (1.52) holds true ; also, from $\hat{\theta} \in A_{N}^{b}$ one gets $\hat{\delta} \in \mathbf{a}_{n}^{b}$.

Again, a suitable gauge fixing of $U$ now yields a unique $\hat{\gamma} \in(-\pi /|\mu|, \pi /|\mu|]^{n}$, and defining $\hat{\gamma}_{s}$ by (1.53), we obtain a bijection

$$
\tilde{\Phi}=P_{2} \times \phi_{r}^{0}: \Omega_{r}^{c}=\mathbf{R}^{2} \times M_{r}^{0} \longrightarrow \hat{\Omega}_{r}^{c}=\mathbf{R}^{2} \times \hat{M}_{r}^{0},\left(\delta_{s}, \gamma_{s} ; \delta, \gamma\right) \longmapsto\left(\hat{\delta}_{s}, \hat{\gamma}_{s} ; \hat{\delta}, \hat{\gamma}\right)
$$

Here we have

$$
\hat{M}^{0} \equiv \mathrm{a}_{n}^{b} \times \mathbb{T}^{n}
$$

with the subscript $r$ on $\hat{\Omega}^{c}$ and $\hat{M}^{0}$ signifying restriction to points where the 
eigenvalue distances of the dual Lax matrix $\hat{A}$ (defined by (2.109), (2.82)) are larger than $2 \tau$; for the $\tilde{\Phi}$-images this property is manifest from the relation

$$
\hat{A}(\hat{P})=\left(U^{*} A U\right)(P), \quad \hat{P} \equiv \tilde{\Phi}(\boldsymbol{P})
$$

Writing the inverse of $\tilde{\Phi}$ as

$$
\tilde{\mathscr{E}}=\boldsymbol{P}_{2} \times \mathrm{b}_{r}^{0}, \quad \mathrm{~b}_{r}^{0} \equiv\left(\phi_{r}^{0}\right)^{-1}
$$

we also prove in Section 2.3 that $b_{r}^{0}$ is essentially an involution. More precisely, taking $\beta=\mu$ one can identify $\hat{M}_{r}^{0}$ and $M_{r}^{0}$ in an obvious way, and then $\mathrm{b}_{r}^{0} \circ \mathrm{b}_{r}^{0}$ is the identity map. (The case $\beta \neq \mu$ is solely a matter of more notation.)

In order to sketch the results of Sections 3.3 and 4.3, we begin by pointing out some crucial differences between the case $\mathrm{III}_{\mathrm{b}}$ and all previous cases (including those studied in I and II ). First, due to the spectral restriction in the definition of $\hat{\Omega}_{r}^{c}$, we are no longer dealing with a manifold that is manifestly connected. However, this is actually the case, as will be shown in Section 4.3. But $\hat{\Omega}_{r}^{c}$ is not simply-connected, as will also be proved in Section 4.3.

Correspondingly, in contrast to all previous cases, where we wind up with a (one-valued) branch $B$ of $R$, we now have to invoke the multi-valued function $R$ to conclude that the bijection $\tilde{\Phi}$ given by $(1.80)$ is a $C^{\omega}$ symplectomorphism when $\Omega_{r}^{c}$ is equipped with $\omega^{c}$ (cf. (1.46)) and $\hat{\Omega}_{r}^{c}$ with $\hat{\omega}^{c}$ (cf. (1.49), (1.50)). To be specific, we introduce the open convex set

$$
\left.\tilde{\Omega} \equiv\left\{(\hat{q}, \hat{\theta}) \in \mathbf{R}^{2 N} \mid \hat{\theta} \in A_{N}^{b}\right\} \quad （ \text { III }_{\mathrm{b}}\right)
$$

and note that $\hat{\Omega}^{c}$ is then obtained from $\tilde{\Omega}$ in the same way as before. Denoting the cover of $\hat{\Omega}_{r}^{c}$ by $\tilde{\Omega}_{r}$, we arrive at a commutative diagram

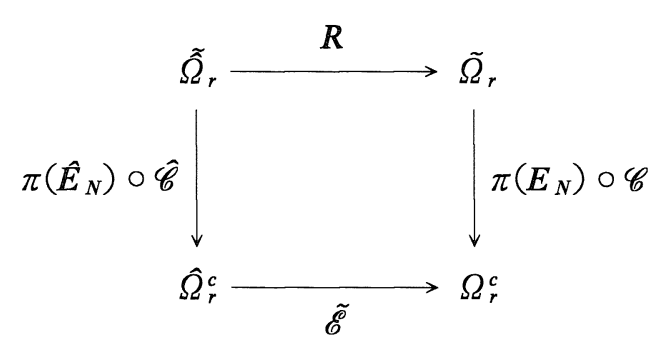

instead of (1.66). However, since we still have $R^{*} \tilde{\omega}=\tilde{\hat{\omega}}$, we reach the conclusion mentioned earlier.

The corresponding conclusion for $\Omega_{r}$ is now obtained by quotienting out the remaining $\mathbf{Z} \times \mathbf{Z}$-action on $\Omega_{r}^{c}$ (recall (1.39) and (1.43)) and the corresponding $\hat{\mathbf{Z}}$ $\times \hat{\mathbf{Z}}_{\text {-action on }} \hat{\Omega}_{r}^{c}$; this gives rise to a commutative diagram 


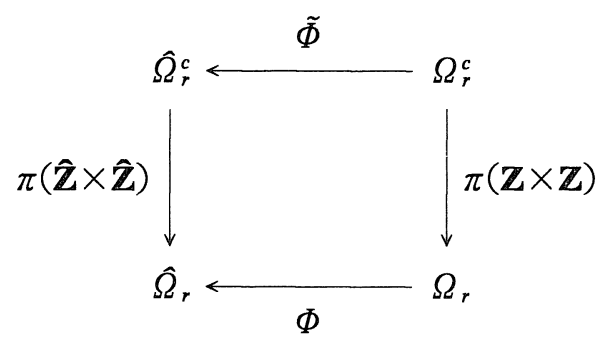

from which the salient features of $\Phi$ can be read off.

As it happens, it would be quite awkward to prove connectedness of the spaces in the above diagrams already in the context of Section 3.3. Therefore, the main result of this section (Theorem 3.5) actually involves certain connected components, whose equality to the spaces in (1.85) will become clear in Section 4.3. Moreover, a consideration of the diagram (1.86) is shifted to Section 4.3 as well.

Just as in the previous cases, we begin Section 4.3 by calculating the reduced map $\phi_{r}^{0}$ explicitly for $n=1$. This enables us to infer that $\phi_{r}^{0}$ admits an extension $\phi^{0}$ to $M^{0}$, provided two new points are added to $\hat{M}^{0}$. This extension gives rise to a non-self-dual situation, but self-duality can be restored by adding two new points to $M^{0}$, too, and by extending $\phi^{0}$ to a map $\phi: M \rightarrow \hat{M}$. The extended spaces $M$ and $\hat{M}$ may and will be viewed as being homeomorphic to the two-sphere, and then $\phi$ is a homeomorphism, cf. Figures 2 and 3 below.

This purely topological extension procedure has an analytic reformulation that greatly enhances its cogency. First, we identify the sphere with radius $R$ in $\mathbb{R}^{3}$ with the projective line $\mathbf{P}^{1}=\mathbb{C} \cup\{\infty\}$ via stereographic projection. Thus we have two patches $\left\{\left(1, z_{1}\right)\right\},\left\{\left(z_{0}, 1\right)\right\}, z_{0}, z_{1} \in \mathbf{C}$, related by the transition function $z \longmapsto$ $1 / z, z \in \mathbf{C}^{*}$. (Here we view $\mathbb{C}$ as $\mathbb{R}^{2}$ via $z \longmapsto(\operatorname{Re} z, \operatorname{Im} z$ ), so the transition functions are $C^{\omega}$ in $\operatorname{Re} z, \operatorname{Im} z$.) Next, we introduce coordinate changes

$$
\hat{\imath}^{f}: \hat{M}^{f 0} \longrightarrow \mathbb{C}^{*}, \quad\left(\hat{\delta}^{f}, \hat{\gamma}^{f}\right) \longmapsto \hat{w}^{f}
$$

where the superscript $f$ signifies that the hat is facultative and where

$$
w \equiv e^{\beta \gamma}\left(\frac{|\mu| \delta-\tau}{\pi-\tau-|\mu| \delta}\right)^{1 / 2}, \quad \hat{w} \equiv e^{\mu \hat{\gamma}}\left(\frac{|\beta| \hat{\delta}-\tau}{\pi-\tau-|\beta| \hat{\delta}}\right)^{1 / 2}
$$

Then the above embedding of $\hat{M}^{f 0}$ in $S^{2}$ may be described by identifying $\left(\hat{\delta}^{f}, \hat{\gamma}^{f}\right)$ with $\left(1, \hat{w}^{f}\right)$. The crux is now that the following holds true.

(i) One has

$$
\hat{\imath}^{f *}\left(\omega_{R}\right)=2 d \hat{\delta}^{f} \wedge d \hat{\gamma}^{f}, \quad R^{2} \equiv \frac{1}{|\beta \mu|}(\pi-2 \tau)
$$

where the symplectic form 


$$
\omega_{R} \equiv 4 R^{2} \frac{d \operatorname{Re} z \wedge d \operatorname{Im} z}{\left[1+(\operatorname{Re} z)^{2}+(\operatorname{Im} z)^{2}\right]^{2}}=2 i R^{2} \frac{d z \wedge d \bar{z}}{\left(1+|z|^{2}\right)^{2}}
$$

amounts to the area form on $S^{2}$. (This is easily verified from (1.88).)

(ii) The map

$$
\phi:\left\langle M, \omega_{R}\right\rangle \longrightarrow\left\langle\hat{M},-\omega_{R}\right\rangle, \quad m \longmapsto \hat{m}
$$

is a $C^{\omega}$ symplectomorphism.

(iii) The compactification of $M^{0}$ just described gives rise to a $C^{\omega}$ extension of the (reduced $n=1$ version of the) Hamiltonian (1.4). Hence one obtains a complete Hamiltonian flow on $M$, as opposed to the flow on $M^{0}$, which is not complete.

(iv) Last but not least, $M$ is a minimal completion, in a sense detailed below.

All of this turns out to admit a generalization to $n>1$. Specifically, (1.87) and (1.88) generalize to

$$
\begin{aligned}
& \hat{\imath}^{f}: \hat{M}^{f 0} \longrightarrow \mathbf{C}^{* n}, \quad\left(\hat{\delta}^{f}, \hat{\gamma}^{f}\right) \longmapsto \hat{w}^{f} \\
& w_{i} \equiv e^{\beta \gamma_{i}}\left(\frac{|\mu| \delta_{i}-\tau}{|\mu| \delta_{0}-\tau}\right)^{1 / 2}, \quad \hat{w}_{i} \equiv e^{\mu \hat{\gamma}_{i}}\left(\frac{|\beta| \hat{\delta}_{i}-\tau}{|\beta| \hat{\delta}_{0}-\tau}\right)^{1 / 2}, \quad i=1, \ldots, n
\end{aligned}
$$

where we have set

$$
\delta_{0} \equiv \frac{\pi}{|\mu|}-\sum_{j=1}^{n} \delta_{j}, \quad \hat{\delta}_{0} \equiv \frac{\pi}{|\beta|}-\sum_{j=1}^{n} \hat{\delta}_{j}
$$

The extension of $\hat{M}^{f 0}$ is now given by

$$
\hat{M}^{f} \simeq \mathbf{P}^{n} \equiv\left(\mathbf{C}^{N} \backslash\{0\}\right) / \mathbf{C}^{*}
$$

Here, we view $\mathbf{P}^{n}$ as a real $2 n$-dimensional $C^{\omega}$ manifold by using the $N$ obvious patches

$\mathscr{P}_{\nu} \equiv\left\{\left(z_{0}, \ldots, z_{n}\right) \in \mathbf{C}^{N} \mid z_{\nu} \neq 0\right\} / \mathbf{C}^{*} \simeq\left\{z \in \mathbf{C}^{N} \mid z_{\nu}=1\right\} \simeq \mathbf{C}^{n}, \nu=0,1, \ldots, n$

whose transition functions are indeed $C^{\omega}$. Then the embedding of $\hat{M}^{f 0}$ in $\mathbf{P}^{n}$ is given by $\left(\hat{\delta}^{f}, \hat{\gamma}^{f}\right) \longmapsto\left(1, \hat{w}^{f}\right) \in \mathscr{P}_{0}$.

Inverting (1.93) yields

$$
|\mu| \delta_{\nu}-\tau=(\pi-N \tau) \frac{\left|w_{\nu}\right|^{2}}{1+\sum_{j=1}^{n}\left|w_{j}\right|^{2}}, \quad \nu=0, \ldots, n, \quad w_{0} \equiv 1
$$




$$
e^{\beta \gamma_{i}}=w_{i} /\left|w_{i}\right|, \quad i=1, \ldots, n
$$

so the first property expressed by (1.89), (1.90) generalizes to

$$
\begin{gathered}
\hat{\imath}^{f *}\left(\omega_{R}\right)=2 \sum_{j=1}^{n} d \hat{\delta}_{j}^{f} \wedge d \hat{\gamma}_{j}^{f}, \quad R^{2} \equiv \frac{1}{|\beta \mu|}(\pi-N \tau) \\
\omega_{R} \equiv \frac{2 i R^{2}}{\sum_{\nu=0}^{n}\left|z_{\nu}\right|^{2}}\left(\sum_{J=1}^{n} d z_{j} \wedge d \bar{z}_{j}-\frac{1}{\sum_{\nu=0}^{n}\left|z_{\nu}\right|^{2}}\left(\sum_{j=1}^{n} \bar{z}_{j} d z_{j}\right) \wedge\left(\sum_{k=1}^{n} z_{k} d \bar{z}_{k}\right)\right) \quad\left(z_{0} \equiv 1\right)
\end{gathered}
$$

Thus, $\omega_{R}$ is a multiple of the global symplectic form derived from the obvious (Fubini-Study) Kähler metric on $\mathbb{P}^{n}$, the multiple being such that the integral of $\omega_{R}$ over a projective line equals $4 \pi R^{2}$. Now the properties (ii)-(iv) hold true for $n>1$, too.

We mention in passing that the starting point for geometric quantization on the Kähler manifold $\left\langle\mathbb{P}^{n}, \omega_{R}\right\rangle$ is the integrality condition

$$
2 R^{2} \in \mathbb{N}^{*}
$$

cf. e.g. [11]. For $N=2$ this is exactly the quantization condition (3.85) in our survey [6], which we imposed for quite different (self-adjointness) reasons, however. For $N>2$ one again needs (1.101) with $R^{2}$ now given by (1.99). (We arrived at the above solution to the classical non-completeness problem after writing [6].)

With the reduced situation under control, it is easy to introduce and study extensions of $\Omega^{c}$ and $\Omega$. Specifically, (1.86) may and will be extended to a commutative diagram

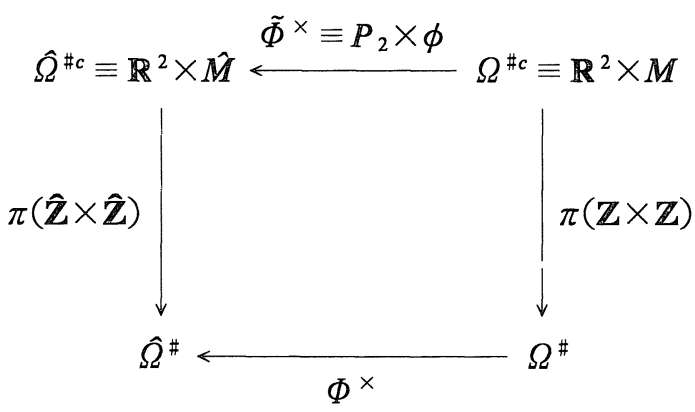

In Section 4.4 we study the reduced map $\phi$. Identifying $M$ and $\hat{M}$ with $\mathbb{P}^{n}$, we may and will view $\phi$ as an involutory antisymplectomorphism of the symplectic manifold $\left\langle\mathbb{P}^{n}, \omega_{\text {ren }}\right\rangle$, where 


$$
\omega_{r e n} \equiv \omega_{R} / 2 R^{2}
$$

This renormalized form equals $2 \pi$ times the Fubini-Study form and is derived from the symplectic form $2 \operatorname{Im}(x, y)$ on $\mathbf{C}^{N} \simeq \mathbf{R}^{2 N}$. (We take the inner product on $\mathbf{C}^{N}$ antilinear in the first slot.) The normalization ensures that for a self-adjoint matrix $A$ the quotient of the unitary group $\exp (-i t A)$ (under the projection $\mathbf{C}^{N} \backslash\{0\} \rightarrow \mathbf{P}^{n}$, cf. (1.95)) equals the Hamiltonian flow $\exp \left(t H_{A}\right)$ on $\left\langle\mathbf{P}^{n}, \omega_{\text {ren }}\right\rangle$, with

$$
H_{A}(z) \equiv(z, A z) /(z, z), \quad z \in \mathbf{C}^{N} \backslash\{0\}
$$

This will be convenient for studying the limit $\tau \uparrow \pi / \mathbf{N}$.

Our main result concerning the map $\phi$ is an immediate consequence of Theorem $4.9: \phi$ is not equal to the quotient of an anti-unitary on $\mathbf{C}^{N}$ for any $\tau \in(0$, $\pi / N)$, whereas for $\tau \uparrow \pi / N$ it does converge to such an anti-automorphism of $\mathbf{P}^{n}$. Specifically, we obtain

$$
\lim _{\tau \uparrow \pi / N} \phi=\mathrm{k} \circ \mathrm{f}_{0}
$$

Here, $k$ and $f_{0}$ are the quotients of complex conjugation and Fourier transformation on $\mathbf{C}^{N}$, cf. (4.108) and (4.128), resp. In contrast, the $\tau \downarrow 0$ limit does not yield a continuous map.

\subsection{Outline of Chapter 5}

The key objects in the construction of the action-angle transform and its harmonic oscillator extension are the matrix $A$ (1.19), the Lax matrices $L$ (given by (2.1), (2.51) and (2.82)), and their duals $\hat{A}$ and $\hat{L}$. The Hamiltonians (1.1)(1.5) are not used anywhere in this construction. They can be viewed as the simplest non-trivial dynamics that arise by taking the trace of a suitable function of $L$. Letting this function vary, we obtain commuting dynamics that are simultaneously diagonalized by the harmonic oscillator transform.

In Chapter 5 we study a class of dynamics obtained in this way, as well as a class of dynamics similarly associated with the dual Lax matrix $\hat{A}$. The latter dynamics are simultaneously diagonalized by the inverse of the harmonic oscillator transform. Since the case $\mathrm{II}_{\mathrm{b}}$ is self-dual, the dual dynamics will not be separately studied.

The dual systems $\hat{\mathrm{II}}_{\mathrm{nr}}$ and $\hat{\mathrm{II}}_{\mathrm{rel}}$ are very different from (1.1) and (1.2), however. In the coordinates $(\hat{x}, \hat{p})$ on the dense submanifold $\hat{\Omega}=\mathbf{T}^{N} \times A_{N}$ (cf. (1.68)) of the extended phase space $\hat{\Omega}^{\#}$ the simplest non-trivial representants read 


$$
D \equiv \sum_{j=1}^{N} \cos \left(|\mu| \hat{x}_{j}\right) V_{j}(\hat{p})
$$

where

$$
\begin{array}{ll}
V_{j} \equiv \prod_{k \neq j}\left(1-\frac{|\mu \mathrm{g}|^{2}}{\left(\hat{p}_{j}-\hat{p}_{k}\right)^{2}}\right)^{1 / 2} & \left(\hat{\mathrm{III}}_{\mathrm{nr}}\right) \\
V_{j} \equiv \prod_{k \neq j}\left(1-\frac{\mathrm{sh}^{2} z}{\operatorname{sh}^{2} \frac{\beta}{2}\left(\hat{p}_{j}-\hat{p}_{k}\right)}\right)^{1 / 2} \quad\left(\hat{\mathrm{II}}_{\mathrm{rel}}\right)
\end{array}
$$

From a physical viewpoint the dual dynamics describe particles on a line, whose distances are bounded below by $|\mu g|$; their momenta vary over the first Brillouin zone $(-\pi /|\mu|, \pi /|\mu|]$. (Admittedly, denoting positions and momenta by $\hat{p}$ and $\hat{x}$, resp., amounts to physical heresy. Introducing additional notation would have its own drawbacks, though ; cf. I for a similar dilemma as concerns the $\Pi_{\mathrm{nr}}$ and $\mathrm{I}_{\text {rel }}$ systems.) The flows generated by the dual dynamics are not complete on $\hat{\Omega}$; at the end of Sections 5.1 and 5.2 we will be in the position to explain how the extension to complete flows on $\hat{\Omega}^{\#}$ can be viewed as a minimal completion.

We now turn to a more detailed sketch of Chapter 5. Section 5.1 begins with an explicit description of the flows associated with $\mathbb{L}$ in terms of the center of mass coordinates $\hat{\delta}_{s}, \hat{\gamma}_{s}$ and harmonic oscillator coordinates $u_{1}, \ldots, v_{n}$. Then we study equilibrium properties of various dynamics. In particular, for the Sutherland dynamics $\tilde{H}$ we show that the points

$$
\left(q_{a}^{e}, 0\right), \quad q_{a, j}^{e} \equiv \frac{|\mu g|}{2}(N+1-2 j)+a, \quad a \in \mathbb{R}, \quad j=1, \ldots, N
$$

are the only equilibria (in agreement with the physical picture).

In Theorem 5.1 we detail a relation between the position part $q(t)$ of the flows and the eigenvalues of a $t$-dependent matrix defined in terms of $A$ and $L$. In Theorem 5.2 we exploit the canonicity of the action-angle map to derive a simple integral representation for the partition functions of a countable set of quotient dynamics on $\Omega$, containing the 'indistinguishable particles on a ring' version $H$ of the Sutherland dynamics, cf. (1.1).

We then study the class of dual dynamics mentioned earlier. Here, the inverse of the harmonic oscillator map is exploited to derive an explicit description of the various flows on $\hat{\Omega}^{\# c}$ in terms of the coordinates $(q, \theta)$ on $\tilde{\Omega}$; the state of affairs on the quotient manifolds $\hat{\Omega}^{\#}$ and $\Omega$ can then be determined via the diagram (1.74).

Theorem 5.3 details a relation of the position part of the dual flows with eigenvalues of a $t$-dependent matrix defined in terms of $\hat{A}$ and $\hat{L}$. As a consequence 
of this relation, the $t \rightarrow \pm \infty$ asymptotics of the spectrum of this matrix yields the long-time asymptotics of the positions. On a distinct-velocity open dense submanifold the spectral asymptotics can be readily determined by invoking Appendix A in $I$.

Correspondingly, Theorem 5.4 shows that the long-time behavior of the dual particles is solitonic : the set of momenta is conserved and the position shifts are factorized in terms of the 2-particle shift. Subsequently, these scattering results are used to clarify the issue of 'minimal completion' mentioned above.

The results for the $\mathrm{II}_{\text {rel }}$ case in Section 5.2 are quite similar to those for the $\mathrm{III}_{\mathrm{nr}}$ case, with Theorems 5.5-5.8 corresponding to the theorems we have just described. Therefore, we refrain from a further discussion. Instead, we wish to draw attention to an important distinction with the previous case.

This concerns the possibility to generalize the dual dynamics (1.106), (1.108) describing $N$ solitons to a dynamics describing $N_{+}<N$ solitons and $N_{-}=N-N_{+}$ antisolitons via the 'crossing' substitution

$$
\hat{p}_{k} \rightarrow \hat{p}_{k}+i \pi / \beta, \quad k=N_{+}+1, \ldots, N
$$

We expect that the resulting $\tilde{\mathrm{I}}_{\text {rel }}$ systems can be handled along lines similar to those followed in II. In particular, the role of the pseudo-self-adjoint Lax matrix from II should be played by a pseudo-unitary matrix, there should be soliton-antisoliton bound states, scattering factorized in terms of the (analytically continued) 2-soliton shift, etc. Using Lemma A.2 below it is not hard to check that the substitution (1.110) (with $p$ replaced by $\theta$ ) in the dual Lax matrix (2.61) indeed yields a pseudo-unitary matrix ; moreover, for $N_{+}, N_{-}=1$ one easily verifies all of the above scenario. We believe however that a reasonably complete study of the general case would be a quite laborious enterprise, even at the purely algebraic level of Chapter 2 and Appendix A in II.

We conclude with a brief sketch of Section 5.3. Again, the harmonic oscillator map enables us to obtain an explicit picture of the flows generated by an extensive class of commuting Hamiltonians. More specifically, oscillation frequencies and equilibrium properties can be read off from the diagonalized flows. Theorems 5.9 and 5.10 may be viewed as generalizations of Theorems 5.1 and 5.2, resp.; they specify position parts in terms of eigenvalues, and partition functions for certain dynamics on $\Omega^{\#}$, resp.

The last two topics of Section 5.3 concern the $\tau \uparrow \pi / N$ limit and the issue of 'minimal completion'. We study these issues only in the reduced context already mentioned below (1.102) ; the non-reduced state of affairs can be readily established from this.

As they stand, all of the above-mentioned Hamiltonians converge to constant functions on $M$ as $\tau \uparrow \pi / N$. (For instance, the Hamiltonian (1.4) has limit 0, since the potential (1.5) does, cf. (1.41).) This may be viewed as a consequence of the 
spectrum of $L$ becoming constant for $\tau \uparrow \pi / N$, cf. (1.76)-(1.79). By means of a suitable renormalization, however, we can ensure non-constant limiting Hamiltonians and, correspondingly, non-trivial limiting flows.

All of the resulting Hamiltonians are of the form (1.104), with $A$ belonging to the maximal abelian algebra of self-adjoint matrices that are diagonalized by Fourier transformation on $\mathbf{C}^{N}$. In particular, the limit of the (renormalized) defining dynamics (1.4) is shown to be proportional to the discrete Laplacean on $\mathbb{C}^{N}$ with periodic boundary conditions. Thus, the map $\mathrm{k} \circ \phi$ may be regarded as a nonlinear generalization of Fourier transformation, in much the same way as the IST for the KdV equation (say). We would like to stress, however, that the $\tau$-value yielding the 'free' dynamics is not $\tau=0$, but $\tau=\pi / N$. (This is not at all evident from (1.4), even with hindsight.)

Our last result is Theorem 5.11, which shows that for $\tau$ near $\pi / N$ the phase space $M \simeq \mathbb{P}^{n}$ may be viewed as a minimal completion of the phase space $M^{0}$ (on which the commuting local flows are not global). The proof makes essential use of the simple limiting behavior of the various flows and maps for $\tau \uparrow \pi / N$, which has already been sketched above.

\section{The Action-angle Transform: Algebraic Aspects}

\subsection{The Case $\mathrm{III}_{\mathrm{nr}}$}

On the space $\tilde{\Omega}$ given by (1.8)-(1.10) the Lax matrix is defined by

$$
L_{j k} \equiv \delta_{j k} \theta_{j}+\left(1-\delta_{j k}\right) \frac{i \mu g}{2 \operatorname{sh} \frac{\mu}{2}\left(q_{j}-q_{k}\right)}, \quad \mu \in i(0, \infty), \quad g<0
$$

Thus the Hamiltonian $\widetilde{H}$ can be written

$$
\widetilde{H}=\frac{1}{2} \operatorname{Tr} L^{2}
$$

cf. (1.1). The fundamental commutation relation reads

$$
\frac{1}{d}[A, L]=e \otimes e-A, \quad d \equiv i \mu g=|\mu g|
$$

where $A=A(q)$ is given by (1.19) and $e$ is the vector with components

$$
e_{j} \equiv e^{\mu q_{j} / 2}, \quad j=1, \ldots, N
$$

Since $L$ is self-adjoint, there exists a unitary $U$ such that 


$$
\hat{L} \equiv U^{*} L U=\operatorname{diag}\left(\hat{\theta}_{1}, \ldots, \hat{\theta}_{N}\right), \quad \hat{\theta}_{N} \leq \cdots \leq \hat{\theta}_{1}
$$

Setting

$$
\begin{aligned}
\hat{A} & \equiv U^{*} A U \\
\hat{f} & \equiv U^{*} e \\
\hat{g} & \equiv U^{t} e
\end{aligned}
$$

the transformed commutation relation can be written

$$
\hat{A}_{j k}\left(d+\hat{\theta}_{k}-\hat{\theta}_{j}\right)=d \hat{f}_{j} \hat{g}_{k}
$$

Upon restriction to the subset

$$
\tilde{\Omega}_{r} \equiv\left\{(q, \theta) \in \tilde{\Omega} \mid \hat{\theta}_{j}-\hat{\theta}_{k} \neq d, \quad j \neq k\right\}
$$

this entails

$$
\hat{A}_{j k}=\hat{f}_{j} C(0, \mu,-g ; \hat{\theta})_{j k} \hat{g}_{k}
$$

where the Cauchy matrix $C$ is given by (A.1). Taking determinants, it follows from Cauchy's identity (A.2) that $\sigma(L)$ is simple and that

$$
\hat{f}_{j} \neq 0, \quad \hat{g}_{j} \neq 0
$$

Next, using $\hat{A}^{-1 *}=\hat{A}$ and $C=\bar{C}$ (the bar denoting complex conjugation), we deduce from (2.11)

$$
\left(C^{-1 t}\right)_{j k}=\left|\hat{f}_{j}\right|^{2} C_{j k}\left|\hat{g}_{k}\right|^{2}
$$

Comparing this to (A.5) and noting $C_{j k} \neq 0$, we get

$$
\left|\hat{f}_{j} \hat{g}_{k}\right|^{2}=\left(l_{j} r_{k}\right)(0, \mu,-g ; \hat{\theta})
$$

Consequently, the inequality (A.7) with $\beta=0$ and $a=\hat{\theta}$ results. Moreover, (A.8) is satisfied in view of $(2.5)$ and simplicity of $\sigma(L)$ on $\tilde{\Omega}_{r}$. Therefore, Lemma A.3 yields $\hat{\theta} \in A_{N}$. As promised below (A.6), one then gets positive quotients in (A.4), so taking positive square roots yields an unambiguous matrix $\widetilde{C}$, cf. (A.6).

Since $\hat{\theta}_{j}-\hat{\theta}_{j+1}>d$ on $\tilde{\Omega}_{r}$, the unitary $U$ is uniquely determined by (2.5) up to right multiplication by a diagonal phase matrix. Before fixing this gauge ambiguity, it is expedient to observe that (2.14) entails the gauge-invariant relations 


$$
\left|\hat{f}_{j}\right|=\xi l_{j}^{1 / 2}, \quad\left|\hat{g}_{j}\right|=\xi^{-1} r_{j}^{1 / 2}, \quad \xi>0
$$

Now $U$ is an isometry, so that

$$
N=\|e\|^{2}=\left\|U^{*} e\right\|^{2}=\|\hat{f}\|^{2}=\xi^{2} \sum_{j=1}^{N} l_{j}=\xi^{2} \sum_{j=1}^{N} \prod_{k \neq j}\left(1-\frac{d}{\hat{\theta}_{j}-\hat{\theta}_{k}}\right) .
$$

We claim that the sum at the rhs equals $N$. To prove this, we need only show that the sum does not depend on $\hat{\theta}$. But this follows just as in the proof of Lemma A.5; in fact, the sum is a degenerate form of $S_{1}$, cf. (A.24). Hence we infer $\xi=1$, so that

$$
\left|\hat{f}_{j}\right|=l_{j}^{1 / 2}, \quad\left|\hat{g}_{j}\right|=r_{j}^{1 / 2}
$$

In order to fix $U$ we now require

$$
\begin{gathered}
\left(U^{*} A U\right)_{k, k+1}<0, \quad k=1, \ldots, N-1 \\
\left(U^{t} e\right)_{1}>0
\end{gathered}
$$

This makes sense, since the quantities at the lhs are nonzero in view of $(2.6),(2.8)$, (2.9) and (2.10). Moreover, (2.18) fixes $U$ up to an overall phase, and then (2.19) fixes this phase.

Next, we set

$$
\hat{\gamma}_{s} \equiv\left(q_{1}+\cdots+q_{N}\right) / N
$$

and introduce

$$
\hat{\gamma}_{1}, \ldots, \hat{\gamma}_{n} \in(-\pi /|\mu|, \pi /|\mu|], \quad \hat{\gamma}_{0}, \hat{\gamma}_{N} \equiv 0
$$

by writing $\hat{g}_{j}$ as (cf. (2.17))

$$
\hat{g}_{j}=\exp \left(-\mu\left[\hat{\gamma}_{j-1}+(j-1) \hat{\gamma}_{s}\right]\right) \prod_{k \neq j}\left(1+\frac{d}{\hat{\theta}_{j}-\hat{\theta}_{k}}\right)^{1 / 2}
$$

It now follows from the above that we must have

$$
\begin{gathered}
\hat{f}_{j}=\exp \left(\mu\left[\hat{\gamma}_{j}+j \hat{\gamma}_{s}\right]\right) \prod_{k \neq j}\left(1-\frac{d}{\hat{\theta}_{j}-\hat{\theta}_{k}}\right)^{1 / 2} \\
\hat{A}_{j k}=\exp \left(\mu\left[\hat{\gamma}_{J}+j \hat{\gamma}_{s}\right]\right) \widetilde{C}(0, \mu,-g ; \hat{\theta})_{j k} \exp \left(-\mu\left[\hat{\gamma}_{k-1}+(k-1) \hat{\gamma}_{s}\right]\right)
\end{gathered}
$$


where $\tilde{\boldsymbol{C}} \in \boldsymbol{S O}(N)$ is the modified Cauchy matrix (A.6). Indeed, $\left|\hat{f}_{1}\right|, \ldots,\left|\hat{f}_{N}\right|$ follow from (2.17), whilst the phases of $\hat{f}_{1}, \ldots, \hat{f}_{N-1}$ follow upon combining (2.11), (2.18) and (2.22). (Note $C_{k, k+1}<0$, cf. (A.1).) Moreover, due to (2.20) one has $|\hat{A}|=|A|=\exp \left(\mu N \hat{\gamma}_{s}\right)$, so the phase of $\hat{f}_{N}$ must be equal to $\exp \left(\mu N \hat{\gamma}_{s}\right)$.

The main reason for our gauge choice can now be made clear: The dual Lax matrix $\hat{A}$ given by (2.24) has a limit for $\hat{\theta}_{k}-\hat{\theta}_{k+1} \downarrow d$ that does not depend on $\hat{\gamma}_{k}$, which will be crucial in Section 4.1. Indeed, in this limit one has $\widetilde{C}_{k, k+1} \rightarrow-1$ and $\widetilde{C}_{j, k+1}, \widetilde{C}_{k l} \rightarrow 0$ for $j \neq k, l \neq k+1$ (in agreement with orthogonality), as is readily checked.

Next, we trade $\hat{\theta} \in A_{N}$ for $\left(\hat{\delta}_{s}, \hat{\delta}\right) \in \mathbf{R} \times \mathrm{a}_{n}$ via $(1.50)$, and $(q, \theta) \in \tilde{\Omega}_{r}$ for $\left(\delta_{s}\right.$, $\left.\gamma_{s} ; \delta, \gamma\right) \in \mathbf{R}^{2} \times M_{r}$ via (1.28)-(1.30). From $\operatorname{Tr} L=\operatorname{Tr} \hat{L}$ and (2.20) we then deduce that (1.52) and (1.53) hold true, resp. Thus, we have now supplied the details of the construction of the map $\tilde{\Phi}$ given by (1.54)-(1.56). (The factorization (1.56) follows from invariance of $\hat{\delta}$ and $\hat{\gamma}$ under shifts $q_{j} \rightarrow q_{j}+q_{0}, \theta_{j} \rightarrow \theta_{j}+\theta_{0}, j$ $=1, \ldots, N$, which is easily established from the above.)

We proceed by showing $\tilde{\Phi}$ is a bijection. To this end we fix $\left(\hat{\delta}_{s}, \hat{\gamma}_{s} ; \hat{\delta}, \hat{\gamma}\right) \in \hat{\Omega}^{c}$ and define $\hat{\theta} \in A_{N}$ via the inverse of (1.50), cf. the rhs of (1.29). Then we define $\hat{L}$ by the rhs of (2.5), $\hat{g}$ and $\hat{f}$ by (2.22) and (2.23), resp., and $\hat{A}$ by (2.24). Since $\hat{A} \in U(N)$, there exists a unitary $V$ such that

$$
V^{*} \hat{A} V=\operatorname{diag}\left(\alpha_{1}, \ldots, \alpha_{N}\right), \quad \alpha \in \mathbf{T}^{N}
$$

Transforming the commutation relation

$$
\frac{1}{d}[\hat{A}, \hat{L}]=\hat{f} \otimes \hat{\mathrm{g}}-\hat{A}
$$

with $V$, we then obtain

$$
\frac{1}{d} L_{j k}^{\prime}\left(\alpha_{j}-\alpha_{k}\right)=e_{l j} e_{r k}-\delta_{j k} \alpha_{k}
$$

where we have set

$$
\begin{aligned}
& L^{\prime} \equiv V^{*} \hat{L} V \\
& e_{l} \equiv V^{*} \hat{f} \\
& e_{r} \equiv V^{t} \hat{g}
\end{aligned}
$$

Taking $j=k$, this says

$$
e_{l j} e_{r j}=\alpha_{j} \neq 0
$$

so that 


$$
e_{l j} \neq 0, \quad e_{r j} \neq 0
$$

Choosing then $j \neq k$, we get $\alpha_{j} \neq \alpha_{k}$, so $\sigma(\hat{A})$ is simple.

We may now conclude that there exists a uniquely determined $x \in F_{N}$ (cf. (1.15)) such that

$$
\sigma(\hat{A})=\left\{e^{\mu x_{1}}, \ldots, e^{\mu x_{N}}\right\}
$$

Next, consider the vectors $q \in W_{N}$ that are on the $\mathbb{Z}$-orbit of $x$. These are given by (1.16), so one has

$$
\sum_{j=1}^{N} x_{j}=\sum_{j=1}^{N} q_{j}+2 \pi(m N-l+1) /|\mu|, \quad m \in \mathbb{Z}, \quad l \in\{1, \ldots, N\}
$$

Thus we can uniquely determine $m$ and $l$, and hence $q \in W_{N}$, by requiring

$$
\sum_{j=1}^{N} q_{j}=N \hat{\gamma}_{s}
$$

Now we are in the position to fix the permutation ambiguity in $V$ by demanding that in (2.25) one has

$$
\alpha_{j}=e^{\mu q}, \quad j=1, \ldots, N
$$

We continue by defining

$$
\theta_{j} \equiv L_{j j}^{\prime}, \quad j=1, \ldots, N
$$

These numbers are real, since $L^{\prime}$ is self-adjoint. Moreover, they are uniquely determined, since the diagonal phase matrix ambiguity left in $V$ only renders the off-diagonal elements of $\mathbb{L}^{\prime}$ ambiguous. Consequently, we obtain a well-defined point $(q, \theta)$ in $\tilde{\Omega}$. Changing variables according to (1.29), (1.30) now yields a map

$$
\tilde{\mathscr{E}}: \mathbb{R}^{2} \times \hat{M}^{0} \longrightarrow \mathbb{R}^{2} \times M, \quad\left(\hat{\delta}_{s}, \hat{\gamma}_{s} ; \hat{\delta}, \hat{\gamma}\right) \longmapsto\left(\delta_{s}, \gamma_{s} ; \delta, \gamma\right)
$$

Lemma 2.1. The map $\tilde{\mathscr{E}}$ is an injection onto $\mathbb{R}^{2} \times M_{r}$ with inverse $\tilde{\Phi}$, which may be factorized as $\mathbb{P}_{2} \times \mathrm{b}^{0}$. The generator

$$
\begin{aligned}
& G:\left(\delta_{s}, \gamma_{s} ; \delta_{1}, \ldots, \delta_{n}, \gamma_{1}, \ldots, \gamma_{n}\right) \longmapsto \\
& \quad\left(\delta_{s}+2 \pi / N|\mu|, \gamma_{s} ; \pi /|\mu|-\sum_{j=1}^{n} \delta_{j}, \delta_{1}, \ldots, \delta_{n-1},-\gamma_{n}, \gamma_{1}-\gamma_{n}, \ldots, \gamma_{n-1}-\gamma_{n}\right)
\end{aligned}
$$


maps $\mathbf{R}^{2} \times M_{r}$ onto itself, and one has

$$
\begin{aligned}
\hat{G} \equiv & \tilde{\Phi} \circ G \circ \tilde{\mathscr{E}}:\left(\hat{\delta}_{s}, \hat{\gamma}_{s} ; \hat{\delta}_{1}, \ldots, \hat{\delta}_{n}, \hat{\gamma}_{1}, \ldots, \hat{\gamma}_{n}\right) \longmapsto \\
& \left(\hat{\delta}_{s}, \hat{\gamma}_{s}+2 \pi / N|\mu| ; \hat{\delta}_{1}, \ldots, \hat{\delta}_{n}, \hat{\gamma}_{1}-2 \pi / N|\mu|, \ldots, \hat{\gamma}_{n}-2 \pi n / N|\mu|\right)
\end{aligned}
$$

Proof. Switching $j \leftrightarrow k$ in (2.27), the modulus of the lhs is invariant, since $L^{\prime}$ is self-adjont. In view of (2.32) this entails that $\left|e_{l j} / e_{r j}\right|$ does not depend on $j$. Since $\left\|e_{l}\right\|^{2}=\|\hat{f}\|^{2}=N$ and $\left\|e_{r}\right\|^{2}=\|\hat{g}\|^{2}=N$, it follows that $\left|e_{l j}\right|=\left|e_{r j}\right|$. Combining this with (2.29)-(2.31) and (2.36), we infer that $V$ can be rendered unique by requiring

$$
e_{l j}=e_{r j}=e^{\mu q_{j} / 2}
$$

Next, we fix a point $\hat{P} \in \hat{\Omega}^{c}$, yielding a point $P \equiv \mathscr{C}^{-1}(\tilde{\mathscr{E}}(\hat{P})) \in \tilde{\Omega}$. We assert that the Lax matrix (2.1), when evaluated in $P$, coincides with $L^{\prime}$. Indeed, in view of (2.37) these matrices have equal diagonals. Using (2.36), (2.41) and (2.27) to express the off-diagonal elements of $L^{\prime}$ in terms of $q_{1}, \ldots, q_{N}$, we obtain the off-diagonal elements in (2.1). Thus our assertion is proved.

As a consequence, the numbers $\hat{\theta}_{1}(\hat{P}), \ldots, \hat{\theta}_{N}(\hat{P})$ are the eigenvalues of $L(P)$. Since they satisfy $\left|\hat{\theta}_{j}-\hat{\theta}_{k}\right| \neq d$, we may infer that $\widetilde{\mathscr{E}}$ maps $\hat{\Omega}^{c}$ into $\mathbf{R}^{2} \times M_{r}$. (In particular, we may conclude at this point that $M_{r}$ is not empty.)

We proceed by observing that $U(P)$ coincides with $V^{*}$. Indeed, from (2.28) and $L^{\prime}=L(P)$ it is evident that $V^{*}$ has the diagonalizing property (2.5) of $U(P)$. Moreover, from (2.25), (2.36) and (2.24) we obtain

$$
\left(V A(P) V^{*}\right)_{k, k+1}=\hat{A}_{k, k+1}<0, \quad k=1, \ldots, N-1
$$

and from (2.30), (2.41) and (2.22) we get

$$
\left(V^{* t} e(P)\right)_{1}=\hat{g}_{1}>0
$$

Thus $V^{*}$ has the three properties that uniquely determine $U(P)$. As a result, we may infer $\tilde{\Phi} \circ \tilde{\mathscr{E}}=\mathrm{id}\left(\hat{\Omega}^{c}\right)$. Arguing similarly for a fixed $P_{0} \in \tilde{\Omega}_{r}$, the matrix $V^{*}(\tilde{\Phi}$ $\left.\left(\mathscr{C}\left(\boldsymbol{P}_{0}\right)\right)\right)$ must equal $U\left(\boldsymbol{P}_{0}\right)$. This yields $\tilde{\mathscr{E}} \circ \tilde{\Phi}=\mathrm{id}\left(\tilde{\Omega}_{r}\right)$, so the first assertion of the lemma now follows.

The map (2.39) amounts to the map (1.12), as anticipated by our (abuse of) notation, cf. (1.28)-(1.30). Now (2.1) entails

$$
L^{G}(P) \equiv L(G(P))=S^{t} L(P) S
$$

where $S$ is the antiperiodic shift (A.35). Therefore, one has $\sigma\left(L^{G}\right)=\sigma(L)$, so $G$ 
maps $\tilde{\Omega}_{r}$ onto itself. Moreover, it follows that $\hat{G}$ leaves $\hat{\delta}_{1}, \ldots, \hat{\delta}_{n}$ invariant. Since the first two coordinates in (2.40) obviously transform as specified, it remains to consider $\hat{\gamma}$.

To this end we observe that the unitary

$$
U^{G}(P) \equiv S^{t} U(P)
$$

satisfies

$$
\begin{aligned}
U^{G}(P)^{*} L(G(P)) U^{G}(P) & =\left(U^{*} L U\right)(P) \\
U^{G}(P)^{*} A(G(P)) U^{G}(P) & =\left(U^{*} A U\right)(P) \\
U^{G}(P)^{t} e(G(P)) & =\left(U^{t} e\right)(P)
\end{aligned}
$$

This entails that $U^{G}(P)$ has the three properties that uniquely determine $U(G(P))$, cf. (2.5), (2.18), (2.19). Hence we must have

$$
U(G(P))=S^{t} U(P)
$$

From (2.47) it now follows that

$$
\hat{A}(\hat{G}(\hat{P}))=\hat{A}(\hat{P})
$$

By virtue of (2.24) and $\hat{\gamma}_{s} \longmapsto \hat{\gamma}_{s}+2 \pi / N|\mu|$, this implies $\hat{\gamma}_{j} \longmapsto \hat{\gamma}_{j}-2 \pi j / N|\mu|$, which completes the proof of (2.40).

\subsection{The Case III $_{\text {rel }}$}

In this case the Lax matrix reads

$$
L_{j k} \equiv \exp \left[\beta\left(\theta_{j}+\theta_{k}\right) / 2\right]\left(V_{j} V_{k}\right)(q)^{1 / 2} \frac{\operatorname{sh} z}{\operatorname{sh}\left(z+\frac{\mu}{2}\left(q_{j}-q_{k}\right)\right)},-i \mu, \beta, z>0
$$

where $V_{j}$ is defined by (1.3). Then $\tilde{H}$ can be written

$$
\tilde{H}=\frac{1}{2} \operatorname{Tr}\left(L+L^{-1}\right)
$$

(To see this, use (A.2). ) Reparametrizing $z$ by (1.7), the commutation relation reads 


$$
\frac{1}{2} \operatorname{cth}(\beta d / 2)[A, L]=e \otimes e-\frac{1}{2}(A L+L A), \quad d=|\mu g|
$$

where

$$
e_{j} \equiv \exp \left(\mu q_{j} / 2+\beta \theta_{j} / 2\right) V_{j}(q)^{1 / 2}
$$

Moreover, $L$ may be written

$$
L_{j k}=e_{j} C(\mu, \beta, g ; q)_{j k} e_{k}
$$

cf. (A.1). Since $q \in W_{N}$, Lemma A.1 entails $L>0$.

Proceeding now as in Section 2.1, we choose a unitary $U$ such that

$$
\hat{L} \equiv U^{*} L U=\operatorname{diag}\left(e^{\beta \hat{\theta}_{1}}, \ldots, e^{\beta \hat{\theta}_{N}}\right), \quad \hat{\theta}_{N} \leq \cdots \leq \hat{\theta}_{1}
$$

Defining $\hat{A}, \hat{f}$ and $\hat{g}$ by (2.6)-(2.8), we then obtain as the generalization of (2.9)

$$
\hat{A}_{j k} \operatorname{sh} \frac{\beta}{2}\left(d+\hat{\theta}_{k}-\hat{\theta}_{j}\right)=\operatorname{sh}(\beta d / 2) e^{-\beta\left(\hat{\theta}_{j}+\hat{\theta}_{k}\right) / 2} \hat{f}_{j} \hat{g}_{k}
$$

On $\tilde{\Omega}_{r}$ (defined by $(2.10)$ ) this can be rewritten

$$
\hat{A}_{j k}=\hat{f}_{j} C(\beta, \mu,-g ; \hat{\theta})_{j k} \hat{g}_{k}
$$

cf. (A.1). Thus, (A.2) again yields non-degeneracy of $\sigma(L)$ and (2.12) follows, too.

Using unitarity of $\hat{A}$ and the properties of $C$ in the same way as before, we now obtain (2.14) with 0 replaced by $\beta$. From this the inequality (A.7) is plain, and since (A.9) is satisfied, we deduce $\hat{\theta} \in A_{N}$ from Lemma A.3. Then (A.6) yields again an unambiguous matrix $\tilde{C} \in S O(N)$.

Once more, (2.15) readily follows, but in the present case it is not easy to determine $\xi$ explicitly. In fact, we are only able to solve this problem at the end of this section (the result being $\xi=\exp [(N-1) z / 2]$ ).

To fix $U$ we may and will impose (2.18) and (2.19), and then we write $\hat{g}$ as

$$
\hat{g}_{j}=\xi^{-1} \exp \left(-\mu\left[\hat{\gamma}_{j-1}+(j-1) \hat{\gamma}_{s}\right]+\beta \hat{\theta}_{j} / 2\right) \prod_{l \neq j}\left(\frac{\operatorname{sh} \frac{\beta}{2}\left(\hat{\theta}_{j}-\hat{\theta}_{l}+d\right)}{\operatorname{sh} \frac{\beta}{2}\left(\hat{\theta}_{j}-\hat{\theta}_{l}\right)}\right)^{1 / 2}
$$

where (2.20), (2.21) are in effect. Then it follows as before that $\hat{f}$ and $\hat{A}$ are given by 


$$
\begin{gathered}
\hat{f}_{j}=\xi \exp \left(\mu\left[\hat{\gamma}_{j}+j \hat{\gamma}_{s}\right]+\beta \hat{\theta}_{j} / 2\right) \prod_{l \neq j}\left(\frac{\operatorname{sh} \frac{\beta}{2}\left(\hat{\theta}_{j}-\hat{\theta}_{l}-d\right)}{\operatorname{sh} \frac{\beta}{2}\left(\hat{\theta}_{j}-\hat{\theta}_{l}\right)}\right)^{1 / 2} \\
\hat{A}_{j k}=\exp \left(\mu\left[\hat{\gamma}_{j}+j \hat{\gamma}_{s}\right]\right) \widetilde{C}(\beta, \mu,-g ; \hat{\theta})_{j k} \exp \left(-\mu\left[\hat{\gamma}_{k-1}+(k-1) \hat{\gamma}_{s}\right]\right)
\end{gathered}
$$

with $\tilde{C} \in S O(N)$ given by (A.6). The limiting behavior of $\hat{A}$ for $\hat{\theta}_{k}-\hat{\theta}_{k+1} \downarrow d$ is now the same as for $\beta=0$, which motivates our gauge fixing.

Mimicking the reasoning in Section 2.1, we again obtain (1.52) and (1.53), the first equality now following from $\exp \left(\beta N \gamma_{s}\right)=|L|=|\hat{L}|=\exp \left(\beta N \hat{\delta}_{s}\right)$, cf. (A.2). Thus we have once again constructed a map $\tilde{\Phi}$ given by $(1.54)-(1.56)$.

To prove $\tilde{\Phi}$ is bijective, we fix $\hat{P} \in \hat{\Omega}^{c}$ and define $\hat{\theta} \in A_{N}$ as before. Also, we define $\hat{L}$ by the rhs of (2.56) and $\hat{A}$ by (2.61). Finally, we define renormalized vectors $\hat{g}_{\rho}, \hat{f}_{\rho}$ via the rhs of (2.59), (2.60) with the factors $\xi^{-1}, \xi$ omitted. Then one readily checks

$$
\frac{1}{2} \operatorname{cth}(\beta d / 2)[\hat{A}, \hat{L}]=\hat{f}_{\rho} \otimes \hat{g}_{\rho}-\frac{1}{2}(\hat{A} \hat{L}+\hat{L} \hat{A})
$$

Now $\hat{A}$ is unitary, so there exists a unitary $V$ obeying (2.25). Then (2.62) yields

$$
L_{j k}^{\prime}\left[\left(\alpha_{j}-\alpha_{k}\right) \operatorname{ch}(\beta d / 2)+\left(\alpha_{j}+\alpha_{k}\right) \operatorname{sh}(\beta d / 2)\right]=2 e_{l j} e_{r k} \operatorname{sh}(\beta d / 2)
$$

where $L^{\prime}$ is defined by (2.28) and where

$$
\begin{aligned}
& e_{l} \equiv V^{*} \hat{f}_{\rho} \\
& e_{r} \equiv V^{t} \hat{g}_{\rho}
\end{aligned}
$$

Now assume $\alpha_{j}=\alpha_{k}$ for $j \neq k$. Then (2.63) implies that the $2 \times 2$ principal minor of $L^{\prime}$ containing the indices $j$ and $k$ is a dyadic, and hence of rank one. But we have $L^{\prime}>0$, a contradiction. Thus $\sigma(\hat{A})$ is simple.

Next, we follow again Section 2.1, writing first $\sigma(\hat{A})$ in terms of a unique $x \in$ $F_{N}$ via (2.33), and then determining a unique $q \in W_{N}$ on its $\mathbb{Z}$-orbit via (2.35). Now we may and will fix the permutation freedom in $V$ by insisting on (2.36). Then (2.63) can be rewritten

$$
L_{j k}^{\prime}=e_{l j} C(\mu, \beta, g ; q)_{j k} e_{r k}
$$

We now introduce $\theta \in \mathbf{R}^{N}$ by setting

$$
L_{j j}^{\prime}=e^{\beta \theta}{ }_{J} V_{j}(q), \quad j=1, \ldots, N
$$


(This makes sense, since $V_{j}$ is positive and $L^{\prime}>0$ implies $L_{j j}^{\prime}>0$.) As before, $L_{j j}^{\prime}$ is gauge-invariant, so after the transformation (1.28)-(1.30) a well-defined map $\tilde{\mathscr{E}}$ given by (2.38) results.

Lemma 2.2. The assertions of Lemma 2.1 hold true in the $\mathrm{III}_{\text {rel }}$ case, too.

Proof. Consider the relation (2.66). Taking determinants, we infer (2.32) holds true. Taking then $j \leftrightarrow k$ and using $\left|M_{j k}\right|=\left|M_{k j}\right|, M=L^{\prime}, \mathrm{C}$, we deduce that there exists $\check{\xi}>0$ such that

$$
\left|e_{l j} / e_{r j}\right|=\check{\xi}^{2}, \quad j=1, \ldots, N
$$

Taking now $j=k$, we obtain

$$
e_{l j} e_{r j}=e^{\mu q}{ }_{J} L_{j j}^{\prime}
$$

so we may fix $V$ by requiring

$$
e_{l j}=\check{\xi} e^{\mu q_{j} / 2}\left(L_{j j}^{\prime}\right)^{1 / 2}, \quad e_{r j}=\breve{\xi}^{-1} e^{\mu q_{J} / 2}\left(L_{j j}^{\prime}\right)^{1 / 2}, \quad \xi>0
$$

The reasoning in the proof of Lemma 2.1 now applies, with (2.43) replaced by

$$
\left(V^{* t} e(P)\right)_{1}=\breve{\xi}\left(V^{* t} e_{r}\right)_{1}=\breve{\xi} \hat{g}_{\rho 1}=\xi \xi \xi(P) \hat{g}_{1}>0
$$

cf. (2.54), (2.70), (2.65) and the definition of $\hat{g}_{\rho}$ above (2.62).

To conclude this section we show that the scale factor $\xi$ is given by

$$
\xi=e^{(N-1) z / 2}
$$

as announced above (2.59). In the process we obtain the remarkable functional equation

$$
F(y, z)=e^{(N-1) z} F(y, 0)
$$

where

$$
F(y, z) \equiv \sum_{i=1}^{N} e^{2 y_{t}} \prod_{j \neq \iota} \frac{\operatorname{sh}\left(y_{i}-y_{j}+z\right)}{\operatorname{sh}\left(y_{i}-y_{j}\right)}
$$

To prove these identities we first observe that 


$$
\xi^{2} F(\beta \hat{\theta} / 2,-z)=\|\hat{f}\|^{2}=\left\|U^{*} e\right\|^{2}=\|e\|^{2}=\operatorname{Tr} L=\operatorname{Tr} \hat{L}=F(\beta \hat{\theta} / 2,0)
$$

where we used (2.60), (2.7), (2.54), (2.51) and (2.56). Similarly, consideration of $\|\hat{g}\|^{2}$ yields

$$
\xi^{-2} F(\beta \hat{\theta} / 2, z)=F(\beta \hat{\theta} / 2,0)
$$

Combining this with (2.75), we obtain the identity

$$
F(y, z) F(y,-z)=F(y, 0)^{2}, \quad y \in \mathbf{C}^{N}, z \in \mathbf{C}
$$

(Indeed, from the above it follows that (2.77) holds for positive $z$ and $2 y \in \beta A_{N}$.)

Next, we fix $y$ in the region $y_{N}<\cdots<y_{1}$ (say) and consider the function

$$
G_{y}(z) \equiv e^{-(N-1) z} F(y, z), \quad z \in \mathbf{C}
$$

Clearly, $G_{y}$ is entire and $2 \pi i$-periodic, and one has

$$
\lim _{\operatorname{Re} z \rightarrow \infty} G_{y}(z)=\sum_{i=1}^{N} e^{2 y_{i}} \prod_{j \neq i} \frac{e^{y_{t}-y_{j}}}{2 \operatorname{sh}\left(y_{i}-y_{j}\right)} \equiv \Lambda(y)
$$

Now $\Lambda(y)$ does not vanish identically, since one clearly has

$$
\lim _{y_{1} \rightarrow \infty} e^{-2 y_{1}} \Lambda(y)=1
$$

Also, using (2.77) we may infer

$$
\lim _{\operatorname{Re} z \rightarrow-\infty} G_{y}(z)=\frac{F(y, 0)^{2}}{\Lambda(y)}
$$

From Liouville's theorem we now deduce $G_{y}(z)=G_{y}(0)$, which entails (2.73). Due to (2.75) we then get (2.72).

\subsection{The Case $\mathrm{II}_{\mathrm{b}}$}

As announced in Section 1.3, we start from the space $\Omega^{c}=\mathbb{R}^{2} \times M^{0}$ in the present case, cf. (1.43), (1.44). Our choice of Lax matrix reads

$$
\begin{aligned}
L\left(\mu, \beta ; \delta_{s}, \gamma_{s}, \delta, \gamma\right)_{j k}= & \exp \left(-\beta\left[\gamma_{j-1}+(j-1) \gamma_{s}\right]\right) \tilde{C}(\mu, \beta, g ; q)_{j k} \\
& \exp \left(\beta\left[\gamma_{k}+k \gamma_{s}\right]\right), \quad i \mu, i \beta, g<0
\end{aligned}
$$


Here, we have $\gamma_{0}, \gamma_{N} \equiv 0, q$ is defined by (1.29), and $\widetilde{C} \in S O(N)$ is defined via (A.6), (A.1) and (A.4). (Since $q \in W_{N}^{b}$, the quotients in (A.4) are indeed positive.) With (1.7) in force, we then obtain

$$
H^{c}=\frac{1}{2} \operatorname{Tr}\left(L+L^{-1}\right)
$$

and the commutation relation reads

$$
\frac{1}{2} \operatorname{cth}(i \beta \mu g / 2)[A, L]=f \otimes g-\frac{1}{2}(A L+L A)
$$

where (cf. (A.4))

$$
\begin{aligned}
& f_{j} \equiv \exp \left(-\beta\left[\gamma_{j-1}+(j-1) \gamma_{s}\right]\right) l_{j}(\mu, \beta, g ; q)^{1 / 2} \\
& g_{j} \equiv \exp \left(\beta\left[\gamma_{j}+j \gamma_{s}\right]\right) r_{j}(\mu, \beta, g ; q)^{1 / 2}
\end{aligned}
$$

Since $L$ is unitary, there exists a unitary $U_{p}$ ( $p$ for provisional) such that

$$
U_{p}^{*} L U_{p}=\operatorname{diag}\left(e^{\beta \hat{p}_{1}}, \ldots, e^{\beta \hat{p}_{N}}\right), \quad-\pi /|\beta|<\hat{p}_{N} \leq \cdots \leq \hat{p}_{1} \leq \pi /|\beta|
$$

Setting

$$
\begin{aligned}
& \hat{A}_{p} \equiv U_{p}^{*} A U_{p} \\
& \hat{f}_{p} \equiv U_{p}^{*} f \\
& \hat{g}_{p} \equiv U_{p}^{t} g
\end{aligned}
$$

we obtain

$$
\hat{A}_{p j k} \operatorname{sh} \frac{\beta}{2}\left(i \mu g+\hat{p}_{k}-\hat{p}_{j}\right)=\operatorname{sh}(i \beta \mu g / 2) e^{-\beta\left(\hat{p}_{J}+\hat{p}_{k}\right) / 2} \hat{f}_{p j} \hat{g}_{p k}
$$

Thus, putting

$$
\Omega_{r}^{c} \equiv\left\{\boldsymbol{P} \in \Omega^{c}\left|\hat{p}_{j}-\hat{p}_{k} \neq\right| \mu g|, 2 \pi /| \beta|-| \mu g \mid, j \neq k\right\}
$$

we have on $\Omega_{r}^{c}$

$$
\hat{A}_{p j k}=\hat{f}_{p j} C(\beta, \mu,-g ; \hat{p})_{j k} \hat{g}_{p k}
$$

We now use (A.2) to deduce $\hat{p}_{N}<\cdots<\hat{p}_{1}$ and

$$
\hat{f}_{p j} \neq 0, \quad \hat{g}_{p j} \neq 0
$$


on $\Omega_{r}^{c}$.

Next, using unitarity of $\hat{A}_{p}$ and the features of $C$ we get

$$
\left|\hat{f}_{p j} \hat{g}_{p k}\right|^{2}=e^{-\beta\left(\hat{p}_{j}+\hat{p}_{k}\right)}\left(l_{j} r_{k}\right)(\beta, \mu,-g ; \hat{p})
$$

From this (A.7) (with $a \rightarrow \hat{p}$ ) follows, and since (A.10) is satisfied on $\Omega_{r}^{c}$, we infer $\hat{p} \in \hat{F}_{N}^{b}$ from Lemma A.3. Moreover, using $|L|=|\hat{L}|$ we conclude

$$
\exp \left(N \beta \gamma_{s}\right)=\exp \left(\beta\left[\hat{p}_{1}+\cdots+\hat{p}_{N}\right]\right)
$$

Now consider the map $y_{1}, \ldots, y_{N} \longmapsto y_{N}+2 \pi /|\beta|, y_{1}, \ldots, y_{N-1}$. Clearly, this map acts bijectively on the set $A_{N}^{b}$ defined by (1.78), so we may use it to generate a $\mathbb{Z}$-action on $A_{N}^{b}$. The set $\hat{F}_{N}^{b}$ may and will be viewed as a fundamental set for this action. Furthermore, in view of (2.96) we can find a unique $\hat{\theta}$ on the orbit of $\hat{p}$ satisfying (1.77). Therefore, we may now switch to a unitary $U$ that satisfies

$$
U^{*} L U=\operatorname{diag}\left(e^{\beta \hat{\theta}_{1}}, \ldots, e^{\beta \hat{\theta}_{N}}\right) \equiv \hat{L}
$$

Next, we define $\hat{A}, \hat{f}$ and $\hat{g}$ by (2.88)-(2.90) with the subscripts $p$ omitted. Then we get (2.91), (2.93)-(2.96) with $p$ 's omitted and with $\hat{p} \rightarrow \hat{\theta}$. We claim that

$$
\left|\hat{f}_{j}\right|=\left|l_{j}(\beta, \mu,-g ; \hat{\theta})\right|^{1 / 2}, \quad\left|\hat{g}_{j}\right|=\left|r_{j}(\beta, \mu,-g ; \hat{\theta})\right|^{1 / 2}
$$

To prove this, we first note that (2.95) entails

$$
\left|\hat{f}_{j}\right|=\xi\left|l_{j}\right|^{1 / 2}, \quad\left|\hat{g}_{j}\right|=\xi^{-1}\left|r_{j}\right|^{1 / 2}, \quad \xi>0
$$

Thus we need only show $\xi=1$. Now we have

$$
\begin{aligned}
\sum_{i=1}^{N} \prod_{j \neq i} \frac{\sin \left(\frac{|\mu|}{2}\left(q_{i}-q_{j}\right)+\tau\right)}{\sin \frac{|\mu|}{2}\left(q_{i}-q_{j}\right)} & =\|f\|^{2}=\left\|U^{*} f\right\|^{2}=\|\hat{f}\|^{2} \\
& =\xi^{2} \sum_{i=1}^{N} \prod_{j \neq i} \frac{\sin \left(\frac{|\beta|}{2}\left(\hat{\theta}_{i}-\hat{\theta}_{j}\right)-\tau\right)}{\sin \frac{|\beta|}{2}\left(\hat{\theta}_{i}-\hat{\theta}_{j}\right)}
\end{aligned}
$$

and when we apply the result (A.23) of Lemma A.5 to the function $S_{1}$, cf. (A.24), we infer that both sums are equal to $\Sigma_{j} \exp (i \tau(N+1-2 j))$. Hence we have $\xi=$ 1 and our claim (2.98) follows. 
To fix the diagonal phase matrix ambiguity left in $U$, we require

$$
\begin{aligned}
& \left(U^{*} A U\right)_{k, k+1}<0, \quad k=1, \ldots, N-1 \\
& e^{-\beta \hat{\theta}_{1} / 2}\left(U^{t} g\right)_{1}>0
\end{aligned}
$$

With the definition

$$
\hat{\gamma}_{s} \equiv \delta_{s}
$$

and the conventions (2.21) in force, we may now define $\hat{\gamma}_{1}, \ldots, \hat{\gamma}_{n}$ by writing $\hat{g}$ as

$$
\hat{g}_{j}=\exp \left(-\mu\left[\hat{\gamma}_{j-1}+(j-1) \hat{\gamma}_{s}\right]+\beta \hat{\theta}_{j} / 2\right) \prod_{l \neq j}\left(\frac{\sin \left(\frac{|\beta|}{2}\left(\hat{\theta}_{j}-\hat{\theta}_{l}\right)+\tau\right)}{\sin \frac{|\beta|}{2}\left(\hat{\theta}_{j}-\hat{\theta}_{l}\right)}\right)^{1 / 2}
$$

Then $\hat{f}$ and $\hat{A}$ are given by

$$
\left.\hat{f}_{j}=\exp \left(\mu \mid \hat{\gamma}_{j}+j \hat{\gamma}_{s}\right]+\beta \hat{\theta}_{j} / 2\right) \prod_{l \neq j}\left(\frac{\sin \left(\frac{|\beta|}{2}\left(\hat{\theta}_{j}-\hat{\theta}_{l}\right)-\tau\right)}{\sin \frac{|\beta|}{2}\left(\hat{\theta}_{j}-\hat{\theta}_{l}\right)}\right)^{1 / 2}
$$

and (2.61), resp., as will be clear by now.

We proceed by trading $\hat{\theta} \in A_{N}^{b}$ for $\left(\hat{\delta}_{s}, \hat{\delta}\right) \in \mathbf{R} \times \mathrm{a}_{n}^{b}$ via (1.50). Then the upshot is, that we have defined a map

$$
\tilde{\Phi}: \Omega_{r}^{c} \longrightarrow \hat{\Omega}^{c}, \quad\left(\delta_{s}, \gamma_{s} ; \delta, \gamma\right) \longmapsto\left(\hat{\delta}_{s}, \hat{\gamma}_{s} ; \hat{\delta}, \hat{\gamma}\right)
$$

where

$$
\hat{\Omega}^{c} \equiv \mathbf{R}^{2} \times \hat{M}^{0}, \quad \hat{M}^{0} \equiv \mathrm{a}_{n}^{b} \times \mathbf{T}^{n}
$$

As before, this map may be written $P_{2} \times \phi_{r}^{0}$, which follows for instance by using the readily verified relation

$U\left(\delta_{s}, \gamma_{s} ; \delta, \gamma\right)_{j k}=\exp \left[-\beta \gamma_{s}(2 j-1) / 2\right] U(0,0 ; \delta, \gamma)_{j k} \exp \left[-\mu \delta_{s}(2 k-1) / 2\right]$

Moreover, comparing (2.61) and (2.82), we obtain the pivotal relation 


$$
\hat{A}=L\left(\beta, \mu ; \hat{\delta}_{s} \hat{\gamma}_{s}, \hat{\delta}, \hat{\gamma}\right)^{t}
$$

(Note that taking $g \rightarrow-g$ in (A.1) and (A.6) amounts to a transposition.)

From this relation it will be clear how to continue : We should go through the same steps as were made to define $\tilde{\Phi}$, in order to construct a map

$$
\tilde{\mathscr{E}}: \hat{\Omega}_{r}^{c} \longrightarrow \Omega^{c}, \quad\left(\hat{\delta}_{s}, \hat{\gamma}_{s} ; \hat{\delta}, \hat{\gamma}\right) \longmapsto\left(\delta_{s}, \gamma_{s} ; \delta, \gamma\right)
$$

Thus, $\hat{\Omega}_{r}^{c}$ is defined as follows : Fixing $\hat{P} \in \hat{\Omega}^{c}$, there exists a unitary $\mathbb{V}_{p}$ such that

$$
V_{p}^{*} \hat{A} V_{p}=\operatorname{diag}\left(e^{\mu x_{1}}, \ldots, e^{\mu x_{N}}\right), \quad-\pi /|\mu|<x_{N} \leq \cdots \leq x_{1} \leq \pi /|\mu|
$$

where $\hat{A}$ is defined by (2.109). Then we set

$$
\hat{\Omega}_{r}^{c} \equiv\left\{\hat{\boldsymbol{P}} \in \hat{\Omega}^{c}\left|x_{j}-x_{k} \neq\right| \beta g|, \quad 2 \pi /| \mu|-| \beta g \mid, \quad j \neq k\right\}
$$

cf. (2.92). Arguing as before, we obtain $x \in F_{N}^{b}$ and using $\exp \left(N \mu \hat{\gamma}_{s}\right)=\exp \left(\mu \Sigma_{j} x_{j}\right)$ we then get a unique $q \in W_{N}^{b}$ on the $\mathbb{Z}$-orbit of $x$, cf. (1.16), (1.36), (1.40). Then we trade $V_{p}$ for a unitary $V$ such that

$$
V^{*} \hat{A} V=\dot{A}(q)
$$

and render $V$ unique by imposing

$$
\begin{aligned}
& \left(V^{*} \hat{L} V\right)_{k+1, k}<0, \quad k=1, \ldots, N-1 \\
& e^{-\mu q_{1} / 2}(V \hat{f})_{1}>0
\end{aligned}
$$

where $\hat{L}$ and $\hat{f}$ are defined via (2.97) and (2.105), resp.

At this stage the rest of the construction of $\tilde{\mathscr{E}}$ will be obvious. But in contrast to the previous cases, it is not obvious that the definition domain $\hat{\Omega}_{r}^{c}$ of $\tilde{\mathscr{E}}$ is non-empty. Among other things, we shall take care of this in the following lemma.

\section{Lemma 2.3. The points}

$$
\hat{P}_{0}\left(\hat{\delta}_{s}, \hat{\gamma}_{s}\right) \equiv\left(\hat{\delta}_{s}, \hat{\gamma}_{s} ; \frac{\pi}{N|\beta|}(1, \ldots, 1), \frac{\pi}{N|\mu|}(n, \ldots, j(N-j), \ldots, n)\right), \quad \hat{\delta}_{s}, \hat{\gamma}_{s} \in \mathbb{R}
$$

belong to $\hat{\Omega}_{r}^{c}$ and the points 
$P_{0}\left(\delta_{s}, \gamma_{s}\right) \equiv\left(\delta_{s}, \gamma_{s} ; \frac{\pi}{N|\mu|}(1, \ldots, 1), \frac{\pi}{N|\beta|}(n, \ldots, j(N-j), \ldots, n)\right), \delta_{s}, \gamma_{s} \in \mathbf{R}$

belong to $\Omega_{r}^{c}$. The map $\tilde{\mathscr{E}}$ is an injection onto $\Omega_{r}^{c}$ with inverse $\tilde{\Phi}$, which may be factorized as $P_{2} \times \mathrm{b}_{r}^{0}$. The generator (2.39) maps $\Omega_{r}^{c}$ onto itself, and (2.40) holds true. The involution

$$
K: \Omega^{c} \longrightarrow \Omega^{c}, \quad\left(\delta_{s}, \gamma_{s} ; \delta, \gamma\right) \longmapsto\left(\delta_{s},-\gamma_{s} ; \delta,-\gamma\right)
$$

maps $\Omega_{r}^{c}$ onto itself, and one has

$$
\begin{aligned}
\hat{K} \equiv \tilde{\Phi} \circ K \circ \tilde{\mathscr{E}}: & \left(\hat{\delta}_{s}, \hat{\gamma}_{s} ; \hat{\delta}_{1}, \ldots, \hat{\delta}_{n}, \hat{\gamma}_{1}, \ldots, \hat{\gamma}_{n}\right) \longmapsto \\
& \left(-\hat{\delta}_{s}, \hat{\gamma}_{s} ; \hat{\delta}_{n}, \ldots, \hat{\delta}_{1},-\hat{\gamma}_{n}, \ldots,-\hat{\gamma}_{1}\right)
\end{aligned}
$$

Finally, identifying $\hat{\Omega}_{r}^{c}(\mu, \beta)$ and $\Omega_{r}^{c}(\beta, \mu)$ in the obvious way, one has

$$
\tilde{\mathscr{E}}(\mu, \beta)=\tilde{\Phi}(\beta, \mu)
$$

the notation being clear from context.

Proof. The Lax matrix (2.82) evaluated in $P_{0}\left(\delta_{s}, \gamma_{s}\right)$ is similar to

$$
\exp \left[\beta \gamma_{s}+i \pi(N+1) / N\right] E_{j k} \omega^{-k}, \quad \omega \equiv e^{2 \pi i / N}
$$

with $E$ given by (A.29), so it follows from (A.2) and Lemma A.4 that $\sigma(L)$ consists of the $N$ th roots of a phase. Thus we obtain

$$
\hat{\delta}_{i}\left(P_{0}\right)=\frac{\pi}{N|\beta|}, \quad i=1, \ldots, n
$$

and $P_{0} \in \Omega_{r}^{c}$. Using (2.108) this argument can be repeated for $\hat{P}_{0}$, yielding

$$
\delta_{i}\left(\hat{P}_{0}\right)=\frac{\pi}{N ! \mu \mid}, \quad i=1, \ldots, n
$$

and $\hat{P}_{0} \in \hat{\Omega}_{r}^{c}$.

The second assertion follows as before by exploiting the commutation relation and the uniqueness of $U$ and $V$, which yields

$$
V(\hat{P})=U(P)^{*}, \quad \hat{P} \equiv \tilde{\Phi}(\boldsymbol{P})
$$

To prove the third claim, we first observe that 


$$
L^{G}(P) \equiv L(G(P))=D_{G}(P) S^{t} L(P) S \overline{D_{G}}(P)
$$

where

$$
D_{G}(P) \equiv e^{\beta\left(\gamma_{n}-\gamma_{s}\right)} \operatorname{diag}\left(e^{N \beta \gamma_{s}}, 1, \ldots, 1\right)
$$

and $S$ is given by (A.35). Therefore, the reasoning below (2.44) applies, and setting

$$
U^{G}(P) \equiv D_{G}(P) S^{t} U(P)
$$

one easily checks (2.46), (2.47) and (2.48) with $e \rightarrow g$. Since $\hat{\theta}$ is invariant, it now follows from uniqueness that

$$
U(G(P))=D_{G}(P) S^{t} U(P)
$$

Then (2.47) entails (2.50), and so (2.40) follows.

The involution $K$ clearly satisfies

$$
A^{K}=A, \quad L^{K}=\bar{L}, \quad g^{K}=A \bar{g}
$$

where $F^{K}$ is defined by $F^{K}(P) \equiv F(K(P))$. Now from $L^{K}=\bar{L}$ one readily deduces

$$
\hat{\theta}(\boldsymbol{K}(\boldsymbol{P}))=\left(-\hat{\theta}_{N}(\boldsymbol{P}), \ldots,-\hat{\theta}_{1}(\boldsymbol{P})\right)
$$

Hence one has $K\left(\Omega_{r}^{c}\right)=\Omega_{r}^{c}$, and $\hat{\delta}_{s}, \hat{\delta}$ transform as specified in (2.119). Thus, to prove (2.119) it remains to show $\hat{\gamma}_{1}, \ldots, \hat{\gamma}_{n} \longmapsto-\hat{\gamma}_{n}, \ldots,-\hat{\gamma}_{1}$.

To this end we invoke Lemma A.7. It enables us to infer that $f$ and $g$ are related by

$$
f=\chi_{N} L A \bar{g}, \quad \chi_{N} \equiv e^{i \tau n}
$$

(To check this, use $L_{j k}=f_{j} C_{j k} g_{k}$.) This relation can now be employed to prove

$$
U^{K}=\chi_{N} \bar{U} \mathscr{R}, \quad U^{K}(P) \equiv U(K(P))
$$

where we have introduced the reversal matrix

$$
\mathscr{R}_{j k} \equiv \delta_{j, N-k+1}
$$

Granting (2.132) for a moment, it follows from $g^{K}=A \bar{g}$ and (2.131) that 


$$
U^{K t} g^{K}=\chi_{N} \mathscr{R} U^{*} A \bar{g}=\mathscr{R} U^{*} L^{-1} f=\mathscr{R} \hat{L}{ }^{-1} \hat{f}
$$

From (2.130), (2.104) and (2.105) one can now read off that $\hat{\gamma}$ transforms as claimed in (2.119).

To prove (2.132), we show that the rhs has the three properties that uniquely determine $U^{K}$. The first property (2.97) is clear from (2.130), and the second one (2.101) follows from $A^{K}=A$ and $\left(\mathscr{R} \hat{A}^{t} \mathscr{R}\right)_{k, k+1}<0$. The third one (2.102) amounts to

$$
e^{\beta \hat{\theta}_{N} / 2}\left(U^{K t} A \bar{g}\right)_{1}>0
$$

Using the equations (2.131), $U^{*} f=\hat{f}$ and (2.105) with $j=N$, it follows that $\chi_{N} \bar{U} \mathscr{R}$ has this property, too, and so (2.132) results.

It remains to prove (2.120). We take $\beta=\mu$ and then suppress the dependence on $\beta, \mu$, the general case being clear from this. The key to proving the involution property $\tilde{\Phi}^{-1}=\tilde{\Phi}$ is the relation (2.109). By uniqueness of $U$ and $V$ it entails we must have

$$
V(\hat{P})=\bar{U}(\hat{P})
$$

On the other hand, (2.124) holds true, so that

$$
U(P)^{t}=U(\hat{P}), \quad \hat{P} \equiv \tilde{\Phi}(\boldsymbol{P})
$$

But now we may deduce (using $\hat{L} \simeq A, A=A^{t}$ )

$$
\begin{gathered}
L\left(\tilde{\Phi}^{2}(P)\right)=\left(U^{*} A U\right)(\hat{P})^{t}=U(P) A(\hat{P}) U(P)^{*}=L(P) \\
A\left(\tilde{\Phi}^{2}(P)\right)=\left(U^{*} L U\right)(\hat{P})=\left(U(P) L(\hat{P})^{t} U(P)^{*}\right)^{t}=A(P) .
\end{gathered}
$$

This entails $\tilde{\Phi}^{2}(P)=P$, since the pair $L, A$ separates the points of $\left\{\left(\delta_{s}, \gamma_{s}\right)\right\} \times M^{0}$.

In Section 3.3 we shall show

$$
\tilde{\Phi}\left(\boldsymbol{P}_{0}\left(\delta_{s}, \gamma_{s}\right)\right)=\hat{\boldsymbol{P}}_{0}\left(\gamma_{s}, \delta_{s}\right)
$$

by invoking the implicit function theorem and analyticity arguments. In fact, for $N$ odd we might also prove (2.140) already at this point, as will transpire from developments below Corollary 4.8. However, this would involve additional notation and a change of viewpoint that could be confusing at this stage. Moreover, for $N$ even the fixed-point arguments we are alluding to do not quite yield (2.140). 


\section{The Action-angle Transform: Analytic and Geometric Aspects}

\subsection{The Case $\mathrm{III}_{\mathrm{nr}}$}

As we have seen in the course of proving Lemma 2.1, the subset $\tilde{\Omega}_{r}$ of $\tilde{\Omega}$ given by (2.10) is non-empty. Alternatively, non-emptiness is obvious from a perturbation argument : Fixing $q \in W_{N}$ and taking $\theta_{j}-\theta_{j+1}=\Lambda d, j=1, \ldots, N-1$, it follows from (2.1) that $\hat{\theta} \in A_{N}$ for $\Lambda$ large enough. Furthermore, $\tilde{\Omega}_{r}$ is an open subset, since $L$ is continuous on $\tilde{\Omega}$. Thus the symplectic form (1.11) may be restricted to $\tilde{\Omega}_{r}$, yielding a symplectic manifold $\left\langle\tilde{\Omega}_{r}, \tilde{\omega}\right\rangle$. We are now prepared to state and prove the main result of this section, already described in Section 1.2.

Theorem 3.1. The map $\tilde{\Phi}$ from Section 2.1 is a $C^{\omega}$ symplectomorphism from $\left\langle\tilde{\Omega}_{r}, \tilde{\omega}\right\rangle$ onto $\left\langle\hat{\Omega}^{c}, \hat{\omega}^{c}\right\rangle$, where $\hat{\omega}^{c}$ is defined by (1.59), (1.60).

Proof. In view of Lemma 2.1 we need only show that $\tilde{\mathscr{E}}$ is a $C^{\omega}$ map satisfying $\tilde{\mathscr{E}}^{*} \tilde{\omega}=\hat{\omega}^{c}$. Just as in $\Pi$, this will be done by constructing and exploiting a commutative diagram, viz., the diagram (1.66). The spaces and maps occurring in (1.66) have been defined above (1.66) and in Section 2.1, except for the map $B$, which has only been described in general terms.

In order to define $B$, we introduce the matrices

$$
\begin{gathered}
\mathscr{A} \equiv \operatorname{diag}\left(\hat{\theta}_{1}, \ldots, \hat{\theta}_{N}\right) \\
\mathscr{L}(\mu, g ; \hat{q}, \hat{\theta})_{j k} \equiv C(0, \mu,-g ; \hat{\theta})_{j k} \eta_{k}
\end{gathered}
$$

with

$$
\eta_{k} \equiv e^{\mu \hat{q}_{k}} \prod_{l \neq k}\left[1+\mu^{2} g^{2} /\left(\hat{\theta}_{k}-\hat{\theta}_{l}\right)^{2}\right]^{1 / 2}
$$

taking at first

$$
(\mu, g, \hat{q}, \hat{\theta}) \in(0, \infty) \times(-\infty, 0) \times \mathbb{R}^{N} \times G_{N} \equiv D_{\mathrm{I}}
$$

where

$$
G_{N} \equiv\left\{y \in \mathbb{R}^{N} \mid y_{N}<\cdots<y_{1}\right\}
$$

We are going to make use of the map

$$
\mathscr{B}_{\mathrm{I}}: D_{\mathrm{I}} \longrightarrow G L(N, \mathrm{C})
$$


which is uniquely determined by the requirements

$$
\begin{gathered}
\mathscr{B}_{\mathrm{I}}^{-1} \mathscr{L}_{\mathrm{I}} \mathscr{B}_{\mathrm{I}}=\operatorname{diag}\left(e^{\mu y_{1}}, \ldots, e^{\mu y_{N}}\right), \quad y \in G_{N} \\
\mathscr{B}_{\mathrm{I}} \zeta=\zeta, \quad \zeta \equiv(1, \ldots, 1)
\end{gathered}
$$

(Here and below, the subscript I signifies that the arguments belong to $D_{\mathrm{I}}$.) In the present case (as opposed to the situation considered in II) it is crucial to take the parameter dependence into account. Arguing as in the proof of II Lemma 3.1 one infers that $\mathscr{B}_{\mathrm{I}}$ is $C^{\omega}$. Consequently, the definitions

$$
\begin{gathered}
q_{\mathrm{I} j} \equiv \mu^{-1} \ln \left(\mathscr{B}_{\mathrm{I}}^{-1} \mathscr{L}_{\mathrm{I}} \mathscr{B}_{\mathrm{I}}\right)_{j j} \\
\theta_{\mathrm{Ij}} \equiv\left(\mathscr{B}_{\mathrm{I}}^{-1} \mathscr{A}_{\mathrm{I}} \mathscr{B}_{\mathrm{I}}\right)_{j j}
\end{gathered}
$$

give rise to a $C^{\omega}$ map

$$
B_{\mathrm{I}}: D_{\mathrm{I}} \longrightarrow G_{N} \times \mathbf{R}^{N}, \quad(\mu, g, \hat{q}, \hat{\theta}) \longmapsto\left(q_{\mathrm{I}}, \theta_{\mathrm{I}}\right)
$$

This map (denoted $\mathscr{E}$ in I ) is in fact canonical (i.e., $B_{\mathrm{I}}^{*} d q_{\mathrm{I}} \wedge d \theta_{\mathrm{I}}=d \hat{q} \wedge d \hat{\theta}$ ), as is proved in I . Our strategy is now to continue $\mu$ to the imaginary axis, yielding a map $B$ that inherits the canonicity property. Specifically, we are going to choose the continuation path $\Gamma$ such that one stays away from the branch varieties and such that the image points move into $\tilde{\Omega}_{r}$. The details now follow.

The path $\Gamma$ is defined by fixing $|\mu|>0$ and $g \in(-\infty, 0)$, setting

$$
\mu(t) \equiv(1-t)|\mu|+i t|\mu|, \quad t \in[0,1]
$$

and taking $\hat{q}_{j}$ and $\hat{\theta}_{j}$ equal to

$$
\begin{gathered}
\hat{q}_{j}^{0} \equiv \pi(N+1-2 j) / N|\mu|, \quad j=1, \ldots, N \\
\hat{\theta}_{j}^{\Lambda} \equiv d(N+1-2 j) \Lambda, \quad j=1, \ldots, N, \quad d \equiv|\mu g|, \quad \Lambda>1
\end{gathered}
$$

resp. Rewriting $\mathscr{L}$ along $\Gamma$ as

$$
\mathscr{L}(t) \equiv \operatorname{diag}\left(\exp \left(\mu(t) \hat{q}_{1}^{0}\right), \ldots, \exp \left(\mu(t) \hat{q}_{N}^{0}\right)\right)+V(t, \Lambda)
$$

it readily follows from (3.2), (3.3) that

$$
\|V(t, \Lambda)\|=O\left(\Lambda^{-1}\right), \quad \Lambda \rightarrow \infty
$$


uniformly along $\Gamma$. (Here and below $\|\bullet\|$ denotes the operator norm derived from the standard inner product on $\mathbf{C}^{N}$.) Therefore, taking $\Lambda \gg 1$ ensures that $\mathscr{L}$ is holomorphic and has simple spectrum in an open neighborhood in $\mathbf{C}^{2+2 N}$ of $\Gamma$. It follows from this that $\mathscr{B}_{\mathrm{I}}$ analytically continues to an open neighborhood in $\tilde{\Omega}$ of the endpoint (which clearly belongs to $\tilde{\Omega}$ ). Moreover, since $|\mathscr{L}(t)|=\exp \left[\mu(t) \sum_{j} \hat{q}_{j}^{0}\right]$ $\neq 0$, the map $B_{\text {I }}$ continues too, cf. (3.9), (3.10). More specifically, we take $\Lambda$ so large that

$$
\left|\tilde{q}_{j}(t)-\hat{q}_{j}^{0}\right|<\pi / 2 N|\mu|, \quad t \in[0,1], \quad j=1, \ldots, N
$$

where $\tilde{q}(t)$ denotes the continuation of $q_{\mathrm{I}}$ along $\Gamma$. This guarantees not only simplicity, cf. (3.13), but also ensures $\tilde{q}(1) \in F_{N}$, as we shall show shortly.

The continuation just detailed yields functions $\mathscr{A}, \mathscr{L}, \mathscr{B}$ and $B$ that are well defined and $C^{\omega}$ on an open convex neighborhood in $\tilde{\Omega}$ of the point $\left(\hat{q}^{0}, \hat{\theta}^{\Lambda}\right)$ with $\Lambda$ $\gg 1$. Choosing a point $\tilde{\hat{P}}$ in this neighborhood, it follows that the matrices $\mathscr{A}$ and $\mathscr{L}$ in $\tilde{\hat{P}}$ are related to $\hat{L}$ and $\hat{A}$ in $\hat{P} \equiv \pi\left(\hat{E}_{N}\right)(\hat{\mathscr{C}}(\tilde{\boldsymbol{P}}))$ via

$$
\begin{gathered}
\mathscr{A}(\tilde{\tilde{P}})=\hat{L}(\hat{\boldsymbol{P}}) \\
\mathscr{L}(\tilde{\tilde{P}})=\left(\hat{D}^{-1} \hat{A} \hat{D}\right)(\hat{P}), \quad \hat{D} \equiv \operatorname{diag}\left(\hat{f}_{1}, \ldots, \hat{f}_{N}\right)
\end{gathered}
$$

(To check this, recall the definitions (3.1)-(3.3) and (2.5), (2.11), (2.22)(2.24).) Furthermore, $\mathscr{A}$ and $\mathscr{L}$ clearly extend to $C^{\omega}$ functions on $\tilde{\Omega}$ satisfying (3.18), (3.19) on all of $\tilde{\Omega}$.

Next, we claim that $\mathscr{B}$ and $B$ can also be analytically continued from the above neighborhood in $\tilde{\Omega}$ to all of $\tilde{\Omega}$, yielding $C^{\omega}$ maps

$$
\begin{gathered}
\mathscr{B}: \tilde{\hat{\Omega}} \longrightarrow G L(N, \mathbf{C}) \\
B: \tilde{\Omega} \longrightarrow \tilde{\Omega}_{r}, \quad(\hat{q}, \hat{\theta}) \longmapsto(\tilde{q}, \tilde{\theta})
\end{gathered}
$$

Here, we have suppressed the dependence on $\mu$, since $\mu$ is now again assumed to be a fixed number in $i(0, \infty)$. Moreover, the image of $(\hat{q}, \hat{\theta})$ under $B$ is provisionally denoted $(\tilde{q}, \tilde{\theta})$; we shall see shortly that $(\tilde{q}, \tilde{\theta})$ equals $(q, \theta)=\left(\mathscr{C}^{-1} \circ \tilde{\mathscr{E}} \circ \pi\left(\hat{E}_{N}\right)\right.$ $\circ \hat{\mathscr{C}})(\hat{q}, \hat{\theta})$, as anticipated in the diagram (1.66).

To prove the above claim, we begin by noting that (3.19) implies $\sigma(\mathscr{L}(\tilde{\tilde{P}}))=$ $\sigma(\hat{A}(\hat{P}))$. Since $\sigma(\hat{A})$ is simple on $\hat{\Omega}^{c}$ (as we have shown in Section 2.1), it follows that $\sigma(\mathscr{L})$ is simple on $\tilde{\Omega}$. But $\tilde{\Omega}$ is a convex subset of $\mathbb{R}^{2 N}$, so that $\mathscr{B}$ and $B$ continue to $C^{\omega}$ functions on $\tilde{\Omega}$ (cf. the proof of II Lemma 3.1). Moreover, $\mathscr{B}$ and $B$ continue to be related by

$$
\mathscr{B}^{-1} \mathscr{L} \mathscr{B}=\operatorname{diag}\left(e^{\mu \tilde{q}_{1}}, \ldots, e^{\mu \tilde{q}_{N}}\right)
$$




$$
\left(\mathscr{B}^{-1} \mathscr{A} \mathscr{B}\right)_{j j}=\tilde{\theta}_{j}
$$

since $\mathscr{B}_{\mathrm{I}}$ and $B_{\mathrm{I}}$ are related in this way, cf. (3.9), (3.10). Therefore, our claim will be proved once we show that $(\tilde{q}, \tilde{\theta})$ belongs to $\tilde{\Omega}_{r}$.

To this end we first recall the formula

$$
\left(V^{*} \hat{A} V\right)(\hat{\boldsymbol{P}})=\operatorname{diag}\left(e^{\mu q_{1}}, \ldots, e^{\mu q_{N}}\right), \quad q \in W_{N}
$$

from Section 2.1, cf. (2.25), (2.36). Now from (3.19) it follows that the numbers at the rhs of (3.22) evaluated in $\hat{P}$ are equal to the phases at the rhs of (3.24), up to an eventual permutation. From (3.17) we may then infer $\tilde{q}(1) \in F_{N} \subset W_{N}$. Moreover, letting $\tilde{\hat{P}}$ vary over $\tilde{\hat{\Omega}}$ and recalling $\tilde{q}$ is continuous on $\tilde{\hat{\Omega}}$, it follows that $\tilde{q}$ must remain real and cannot cross the hyperplanes bounding $W_{N}$. (Indeed, these hyperplanes correspond to eigenvalue collisions.) Therefore, $\tilde{q}$ belongs to $W_{N}$. Since $A(\tilde{q})$ and $A(q)$ are related by a permutation, it now follows that $q$ and $\tilde{q}$ are on the same Z-orbit in $W_{N}$. (Cf. the paragraph following (1.18).) But we also have $\Sigma \tilde{q}_{j}=\sum \hat{q}_{j}$ (by analytic continuation) and $\Sigma q_{j}=N \delta_{s}=N \hat{\gamma}_{s}=\Sigma \hat{q}_{j}$, so that $\sum \tilde{q}_{j}=\Sigma q_{j}$.

Consequently, we must have $\tilde{q}=q$. From this we deduce

$$
\mathscr{B}(\tilde{\tilde{P}})=\hat{D}(\hat{P})^{-1} V(\hat{P}) D(\tilde{\tilde{P}})
$$

where $D$ is an invertible diagonal matrix. (In fact, one has $D_{j j}=\exp \left(\mu q_{j} / 2\right)$ ), as is easily verified.) But now we may conclude that the vector $\tilde{\theta}$ in (3.23) equals the vector $\theta$ in (2.37), cf. (2.28). Hence, we have $(\tilde{q}, \tilde{\theta})=(q, \theta) \in \tilde{\Omega}_{r}$, so the above claim is now proved.

In the process of proving the claim we have also defined $B$ and shown that the diagram (1.66) commutes. Since $B$ is a $C^{\omega}$ map satisfying (1.62), it follows from commutativity that $\tilde{\mathscr{E}}$ is a $C^{\omega}$ symplectomorphism.

Thus far we have restricted our considerations to the open submanifold $\tilde{\Omega}_{r}$ of $\tilde{\Omega}$. Recalling the definition (2.10), we see that the boundary set

$$
\tilde{\Omega}_{b} \equiv \tilde{\Omega} \backslash \tilde{\Omega}_{r}
$$

is equal to the zero locus of the function

$$
F \equiv \prod_{1 \leq j<k \leq N}\left[\left(\lambda_{j}-\lambda_{k}\right)^{2}+\mu^{2} g^{2}\right]
$$

where $\lambda_{1}, \ldots, \lambda_{N}$ are the roots of $\left|L(q, \theta)-\lambda \mathbf{1}_{N}\right|$. Since $F$ is invariant under permutations of $\lambda_{1}, \ldots, \lambda_{N}$, it is a polynomial in the symmetric functions of $L$. But $L$ is $C^{\omega}$ on $\tilde{\Omega}$, so it follows that $F$ is $C^{\omega}$ on $\tilde{\Omega}$, too. Therefore, $\tilde{\Omega}_{b}$ is a subvariety of 
codimension at least one. As a consequence, $\tilde{\Omega}_{r}$ is a dense full measure submanifold of $\tilde{\Omega}$. In particular, we may now deduce that $L$ has simple spectrum with minimal eigenvalue distance $\geq d$ on all of $\tilde{\Omega}$. (Recall that $\hat{\theta} \in A_{N}$ on $\tilde{\Omega}_{r}$, i.e., the eigenvalue distance is $>d$ on $\tilde{\Omega}_{r .}$ )

With Theorem 3.1 at our disposal, we can settle two obvious questions about $\tilde{\Omega}_{b}$. First, we may infer that $\tilde{\Omega}_{b}$ is non-empty. Indeed, Theorem 3.1 entails that $\tilde{\Omega}_{r}$ and $\hat{\Omega}^{c}$ are symplectically diffeomorphic, so a fortiori these manifolds are homeomorphic. Therefore, we have

$$
\pi_{1}\left(\tilde{\Omega}_{r}\right)=\pi_{1}\left(\hat{\Omega}^{c}\right)=\pi_{1}\left(\tilde{\Omega} / \hat{E}_{N}\right)=\hat{E}_{N}
$$

where the last equality follows from the convexity of $\tilde{\Omega}$. Since $\tilde{\Omega}$ is convex, too, $\tilde{\Omega}_{r}$ is smaller than $\tilde{\Omega}$, as claimed. Second, we may deduce that $\tilde{\Omega}_{b}$ does not separate $\tilde{\Omega}_{r}$ into several connected components (as it might, a priori). Indeed, $\hat{\Omega}^{c}$ is connected, so $\tilde{\Omega}_{r}$ must be connected as well.

In Section 4.1 we shall greatly improve on these observations by detailing the structure of $\tilde{\Omega}_{b}$ in regard to $\tilde{\Omega}_{r}$. In particular, it will be shown that $\tilde{\Omega}_{b}$ actually has codimension 2 .

We close this section by studying the two quotients described by the covering sequence (1.27). First of all, it should be recalled that the $\mathbb{Z}$-action on $\tilde{\Omega}$ is isospectral w.r.t. $L$, cf. (2.44). Therefore it leaves $\tilde{\Omega}_{r}$ and $\tilde{\Omega}_{b}$ invariant, and so we obtain open dense full measure submanifolds

$$
\Omega_{r}^{\prime} \equiv \tilde{\Omega}_{r} / \mathbb{Z}^{\prime}, \quad \Omega_{r} \equiv \tilde{\Omega}_{r} / \mathbb{Z}
$$

of $\Omega^{\prime}$ and $\Omega$, resp. Next, we recall (2.39) and (2.40). From these formulas we read off

$$
\begin{array}{ll}
\hat{G}^{N}\left(\hat{\delta}_{s}, \hat{\gamma}_{s} ; \hat{m}\right)=\left(\hat{\delta}_{s}, \hat{\gamma}_{s}+2 \pi /|\mu| ; \hat{m}\right), & \hat{m} \in \hat{M}^{0} \\
G^{N}\left(\delta_{s}, \gamma_{s} ; m\right)=\left(\delta_{s}+2 \pi /|\mu|, \gamma_{s} ; m\right), & m \in M_{r}
\end{array}
$$

Hence, quotienting out the $\mathbb{Z}^{\prime}$-action amounts to letting the center of mass position $\delta_{s}$ vary over $S^{1}$ instead of $\mathbb{R}$, in keeping with the physical picture sketched in Section 1.1. Since the quotient respects the direct product structure of the center of mass space and the reduced space, and since it acts trivially on the latter, results for this situation are immediate from Theorem 3.1, and correspondingly we shall not spell these out.

The situation on $\Omega_{r}$ is more interesting, in as much as the quotient now mixes the center of mass and internal spaces. Consider the top line of the diagram (1.67). The map $\tilde{\Phi}$ is a bijection mapping orbits of the $\mathbb{Z}$-action on $\tilde{\Omega}_{r}$ onto orbits of the $\hat{\mathbb{Z}}$-action on $\hat{\Omega}^{c}$ (cf. (2.39), (2.40)). Thus we may and will define the map $\Phi$ in the 
bottom line in such a way that the diagram commutes. The space $\hat{\Omega}$ may and will be viewed as being obtained from $\tilde{\hat{\Omega}}=\mathbf{R}^{N} \times A_{N}$ by factoring out the $\hat{\mathbf{Z}}^{N}$-action (1.65) on $\mathbf{R}^{N}$, which yields (1.68)-(1.70). We are now prepared for the last result of this section.

Corollary 3.2. The map $\Phi$ is a symplectomorphism from $\left\langle\Omega_{r}, \omega\right\rangle$ onto $\langle\hat{\Omega}, \hat{\omega}\rangle$.

Proof. This follows from Theorem 3.1, the above definitions and the commutative diagram (1.67).

\subsection{The Case $\mathrm{III}_{\text {rel }}$}

Just as in the case $\mathrm{III}_{\mathrm{nr}}$, the set $\tilde{\Omega}_{r}$ given by (2.10) is a non-empty open subset of $\tilde{\Omega}$, so that $\left\langle\tilde{\Omega}_{r}, \tilde{\omega}\right\rangle$ is a symplectic manifold. However, in this case there appears to be no obvious analytic argument from which non-emptiness follows. (Recall we proved non-emptiness by purely algebraic means in Section 2.2.)

Theorem 3.3. The assertion of Theorem 3.1 holds true for the map $\tilde{\Phi}$ from Section 2.2.

Proof. The proof runs parallel to the proof of Theorem 3.1. To define the map $B$ in the diagram (1.66) we replace (3.1)-(3.3) by the functions

$$
\begin{gathered}
\mathscr{A} \equiv \operatorname{diag}\left(e^{\beta \hat{\theta}_{1}}, \ldots, e^{\beta \hat{\theta}_{N}}\right) \\
\mathscr{L}(\beta, \mu, g ; \hat{q}, \hat{\theta})_{j k} \equiv C(\beta, \mu,-g ; \hat{\theta})_{j k} \eta_{k} \\
\eta_{k} \equiv e^{\mu \hat{q}_{k}} \prod_{l \neq k}\left[1+\sin ^{2}\left(\frac{1}{2} \beta \mu g\right) / \operatorname{sh}^{2} \frac{\beta}{2}\left(\hat{\theta}_{k}-\hat{\theta}_{l}\right)\right]^{1 / 2}
\end{gathered}
$$

from II Section 3B. Thus $\beta$ and $\mu$ are positive numbers, and we take at first $(\hat{q}, \hat{\theta})$ $\in \mathbf{R}^{N} \times G_{N}$, cf. (3.5), and $g \in(-\pi / \beta \mu, 0)$. As before, the set of $(\beta, \mu, g, \hat{q}, \hat{\theta})$ thus obtained will be denoted by $D_{\mathrm{I}}$. Again, our starting point is the map (3.6) determined by (3.7), (3.8). Arguing as above, this map gives rise to a $C^{\omega}$ map

$$
B_{\mathrm{I}}: D_{\mathrm{I}} \longrightarrow G_{N} \times \mathbf{R}^{N}, \quad(\beta, \mu, g, \hat{q}, \hat{\theta}) \longmapsto\left(q_{\mathrm{I}}, \theta_{\mathrm{I}}\right)
$$

which is now defined by

$$
q_{\mathrm{I} j} \equiv \mu^{-1} \ln \alpha_{j}, \quad \alpha_{j} \equiv\left(\mathscr{B}_{\mathrm{I}}^{-1} \mathscr{L}_{\mathrm{I}} \mathscr{B}_{\mathrm{I}}\right)_{j j}
$$


$\theta_{\mathrm{I} j} \equiv \beta^{-1} \ln \left(\mathscr{B}_{\mathrm{I}}^{-1} \mathscr{A}_{\mathrm{I}} \mathscr{B}_{\mathrm{I}}\right)_{j j}-\frac{1}{2 \beta} \ln \left(\prod_{k \neq j} \frac{\left(e^{i \tau} \alpha_{j}-e^{-i \tau} \alpha_{k}\right)\left(e^{-i \tau} \alpha_{j}-e^{i \tau} \alpha_{k}\right)}{\left(\alpha_{j}-\alpha_{k}\right)^{2}}\right)$

Here, all logarithms may and will be chosen real, and $B_{I}$ thus defined is a canonical transformation, as proved in I . (Indeed, $B_{I}$ coincides with the map $\mathscr{E}$ of the $\Pi_{\text {rel }}$ regime.)

Next, we continue $\mathscr{A}_{\mathrm{I}}, \mathscr{L}_{\mathrm{I}}, \mathscr{B}_{\mathrm{I}}$ and $B_{\mathrm{I}}$ along a path $\Gamma$ that is here defined as follows. We fix $\beta,|\mu|>0$ and take

$$
g \in(-\varepsilon \pi / N|\beta \mu|, 0) \equiv I_{\varepsilon}, \quad \varepsilon \in(0,2]
$$

where $\varepsilon$ is yet to be chosen. Now we take $\mu(t)$ and $\hat{q}$ equal to (3.12) and (3.13), resp., whereas $\hat{\theta}_{j}$ is taken equal to

$$
\hat{\theta}_{j}^{0} \equiv \pi(N+1-2 j) / N|\beta|, \quad j=1, \ldots, N
$$

As the analog of (3.15) we now set

$$
\mathscr{L}(t) \equiv \operatorname{diag}\left(\exp \left(\mu(t) \hat{q}_{1}^{0}\right), \ldots, \exp \left(\mu(t) \hat{q}_{N}^{0}\right)\right)+V(t, g)
$$

Then we infer from (3.33), (3.34) that

$$
\|V(t, g)\|=O(\varepsilon), \quad \varepsilon \rightarrow 0
$$

uniformly on $[0,1] \times I_{\varepsilon}$. Hence, by taking $\varepsilon \ll 1$ we can ensure that $\mathscr{L}$ is holomorphic and $\sigma(\mathscr{L})$ simple in a $\mathbf{C}^{3+2 N}$-neighborhood of $\Gamma$. In fact, we may and will require that $\varepsilon$ be sufficiently small so that (3.17) holds. This entails

$$
0<\left|\mu(t)\left[\tilde{q}_{j}(t)-\tilde{q}_{k}(t)+i \beta g\right]\right|<2 \pi, \quad t \in[0,1]
$$

(Use (3.12), (3.13) and (3.38) to check this.) Therefore, the argument of the second logarithm in (3.37) stays away from 0 along $\Gamma$. But then the argument of the first one does so, too, cf. II (3.63)-(3.67).

As a result, we obtain functions $\mathscr{A}, \mathscr{L}, \mathscr{B}$ and $B$ that are $C^{\omega}$ in $(g, \hat{q}, \hat{\theta})$ for $g \in I_{\varepsilon}$ and $(\hat{q}, \hat{\theta})$ varying over an open convex neighborhood in $\tilde{\hat{\Omega}}$ of the point $\left(\hat{q}^{0}\right.$, $\hat{\theta}^{0}$ ). (A priori, this neighborhood depends on $g$.) Again, the matrices $\mathscr{A}$ and $\mathscr{L}$ are related to the matrices $\hat{L}$ and $\hat{A}$ via (3.18) and (3.19), resp. (To see this, compare (3.32)-(3.34) and (2.56), (2.58), (2.59)-(2.61).) It is also clear by inspection that $\mathscr{A}$ and $\mathscr{L}$ admit a further continuation to all of $\tilde{\Omega}$ and to any $g \in(-\infty, 0)$, and that (3.18), (3.19) continue to hold.

Proceeding as before, we now claim that $\mathscr{B}$ and $B$ can be continued to all of $\tilde{\Omega}$ 
and to any $g \in(-\infty, 0)$, yielding $C^{\omega}$ maps (3.20), (3.21) when the dependence on the parameters is again suppressed. To prove this claim, we first note that in view of (3.19) the spectrum of $\mathscr{L}$ on $\tilde{\hat{\Omega}}$ is simple and belongs to the unit circle. (Indeed, this holds for $\hat{A}$ on $\hat{\Omega}^{c}$, cf. Section 2.2.) Also, since $i \tau>0$ in the case at hand, the argument of the second logarithm in the continuation of (3.37) cannot vanish on $\tilde{\Omega}$. Since $\tilde{\Omega}$ is convex, we may now deduce the continuation property by repeating arguments detailed in $I$, cf. II Lemma 3.1 and II (3.63)-(3.67).

To finish the proof of the above claim, we show that $B$ maps into $\tilde{\Omega}_{r}$. Following the reasoning for the previous case, (3.36) again leads to (3.22), whilst (3.37) entails

$$
\left(\mathscr{B}^{-1} \mathscr{A} \mathscr{B}\right)_{j j}(\tilde{\tilde{P}})=e^{\beta \tilde{\theta}_{J}} V_{j}(\tilde{q})^{1 / 2}
$$

Now (3.24) is still valid, so it follows as before that the two vectors $\tilde{q}$ and $q$ are equal and that (3.25) holds true. From (3.25) we now deduce that the vector $\tilde{\theta}$ in (3.43) and the vector $\theta \in \mathbf{R}^{N}$ in (2.67) are equal $\bmod 2 \pi i / \beta$, so it remains to show that the former vector is real.

To this end we recall the defining properties (3.7), (3.8) of $\mathscr{B}_{\mathrm{I}}$, the definition (3.33) of $\mathscr{L}$, and our choice (3.13) of $\hat{q}$. From this we readily infer that $\mathscr{B} \rightarrow \mathbf{1}_{N}$ along $\Gamma$ as $g \uparrow 0$. But then the continuation of (3.37) cannot lead to a non-zero multiple of $2 \pi i / \beta$, so that $\tilde{\theta}$ is real. Thus, our claim now follows, first for small $g$ and then for any $g \in(-\infty, 0)$ via analytic continuation. The last paragraph of the proof of Theorem 3.1 now applies verbatim, completing the proof.

In the present case the boundary set (3.26) equals the zero locus of

$$
F \equiv \prod_{1 \leq j<k \leq N}\left[\left(\lambda_{j}-\lambda_{k}\right)^{2}-4 \lambda_{j} \lambda_{k} \operatorname{sh}^{2} z\right]
$$

Hence it follows as before that $\operatorname{codim} \tilde{\Omega}_{b} \geq 1$ and that $\tilde{\Omega}_{r}$ is a dense full measure submanifold of $\tilde{\Omega}$. Moreover, $L$ has positive and simple eigenvalues satisfying $\left|\ln \lambda_{j}-\ln \lambda_{k}\right| \geq 2 z, j \neq k$, on all of $\tilde{\Omega}$. Finally, since (3.28) is still valid, it follows once again that $\tilde{\Omega}_{b}$ is non-empty and does not disconnect $\tilde{\Omega}$. Among other things, we will sharpen these results in Section 4.2 by proving codim $\tilde{\Omega}_{b}=2$.

Since (2.44) still holds in the case at hand, it follows that $\tilde{\Omega}_{r}$ and $\tilde{\Omega}_{b}$ are left invariant by the $\mathbf{Z}$-action on $\tilde{\Omega}$. Thus, the definitions (3.29) again make sense. Since (3.30), (3.31) hold as well (cf. Lemma 2.2), the observations below these equations apply again. In particular, Theorem 3.3 has the following corollary.

Corollary 3.4. The assertion of Corollary 3.2 holds true in the $\mathrm{III}_{\text {rel }}$ case, too. 


\subsection{The Case $\mathrm{III}_{\mathrm{b}}$}

It is convenient to begin this section by introducing

$$
\hat{\Omega}_{b}^{f c} \equiv \hat{\Omega}^{f c} \backslash \hat{\Omega}_{r}^{f c}
$$

where $f$ signifies that the hat is facultative, and

$$
\begin{aligned}
& F \equiv \prod_{1 \leq j<k \leq N}\left[\left(\lambda_{j}-\lambda_{k}\right)^{2}+4 \lambda_{j} \lambda_{k} \sin ^{2} \tau\right] \\
& \hat{F} \equiv \prod_{1 \leq j<k \leq N}\left[\left(\alpha_{j}-\alpha_{k}\right)^{2}+4 \alpha_{j} \alpha_{k} \sin ^{2} \tau\right]
\end{aligned}
$$

where the $\lambda_{j}$ and $\alpha_{j}$ are the roots of the characteristic polynomial of $L(P), P \in \Omega^{c}$, and $\hat{A}(\hat{P}), \hat{P} \in \hat{\Omega}^{c}$, resp. Then the boundary sets $\Omega_{b}^{c}$ and $\hat{\Omega}_{b}^{c}$ are equal to the zero loci of $F$ and $\hat{F}$, resp. Now we have seen in Lemma 2.3 that the set $\hat{\Omega}_{r}^{f c}$ is a non-empty subset of $\hat{\Omega}^{f c}$. Consequently, $\hat{F}^{f}$ does not vanish identically on $\hat{\Omega}^{f c}$, and so $\hat{\Omega}_{b}^{f c}$ is a subvariety of codimension at least one. Therefore, $\hat{\Omega}_{r}^{f c}$ is an open dense full measure submanifold of $\hat{\Omega}^{f c}$. This entails in particular that the minimal distance between the $\lambda_{j}$ in arclength along the unit circle equals $2 \tau$.

In Section 4.3 we shall obtain a quite detailed picture of the boundary sets. As it turns out, these subvarieties have codimension two, so they do not disconnect $\hat{\Omega}^{c}$ and $\Omega^{c}$. However, for the time being we have to phrase the following theorem in terms of connected components. Specifically, we denote the component of $\hat{\Omega}_{r}^{c}$ containing the point $\hat{P}_{0}(0,0)$ (given by $(2.116)$ ) by $\hat{\Omega}_{r 0}^{c}$, and we set

$$
\Omega_{r 0}^{c} \equiv \tilde{\mathscr{E}}\left(\hat{\Omega}_{r 0}^{c}\right)
$$

Theorem 3.5. One has

$$
\tilde{\mathscr{E}}\left(\hat{\boldsymbol{P}}_{0}(0,0)\right)=P_{0}(0,0)
$$

where $P_{0}(0,0)$ is given by (2.117). The space $\Omega_{r 0}^{c}$ is equal to the connected component of $\Omega_{r}^{c}$ that contains $P_{0}(0,0)$. The map $\tilde{\Phi}$ is a $C^{\omega}$ symplectomorphism from $\left\langle\Omega_{r 0}^{c}, \omega^{c}\right\rangle$ onto $\left\langle\hat{\Omega}_{r 0}^{c}, \hat{\omega}^{c}\right\rangle$, where $\omega^{c}$ is given by (1.46) and $\hat{\omega}^{c}$ by (1.59), (1.60).

Proof. We begin by continuing the functions $\mathscr{A}_{\mathrm{I}}, \mathscr{L}_{\mathrm{I}}, \mathscr{B}_{\mathrm{I}}$ and $B_{\mathrm{I}}$ defined in the proof of Theorem 3.3 to the $\mathbb{I I}_{\mathrm{b}}$ regime. To this end we choose a path $\Gamma$ by taking $\hat{q}$ and $\hat{\theta}$ equal to $\hat{q}^{0}$ and $\hat{\theta}^{0}$, resp. (cf. (3.13), (3.39)), fixing $|\mu|,|\beta|>0$ and $g$ as in (3.38), and defining $\mu(t)$ by (3.12) and $\beta(t)$ by

$$
\beta(t) \equiv(1-t)|\beta|+i t|\beta|, \quad t \in[0,1]
$$


Then the endpoint of $\Gamma$ has image $\hat{P}_{0}(0,0)$ under $\pi\left(\hat{E}_{N}\right) \circ \hat{\mathscr{C}}$, and (3.40), (3.41) hold true again. Thus we may and will choose $\varepsilon$ so that (3.17) is valid, and then (3.42) with $\beta$ replaced by $\beta(t)$ follows as before.

As a consequence we obtain functions $\mathscr{A}, \mathscr{L}, \mathscr{B}$ and $B=(\tilde{q}, \tilde{\theta})$ that are $C^{\omega}$ in $(g, \hat{q}, \hat{\theta})$ for $g \in I_{\varepsilon}$ and $(\hat{q}, \hat{\theta})$ varying over an open convex neighborhood $N_{g} \subset \hat{\Omega}$ of $\left(\hat{q}^{0}, \hat{\theta}^{0}\right)$. On $N_{g}$ these functions satisfy

$$
\begin{gathered}
\mathscr{B}^{-1} \mathscr{L} \mathscr{B}=\operatorname{diag}\left(e^{\mu \tilde{q}_{1}}, \ldots, e^{\mu \tilde{q}_{N}}\right), \quad \mathscr{B} \zeta=\zeta \\
\left(\mathscr{B}^{-1} \mathscr{A} \mathscr{B}\right)_{j j}=e^{\beta \tilde{\theta}_{J}} V_{j}^{b}(\tilde{q})
\end{gathered}
$$

due to (3.7), (3.8), (3.36) and (3.37). Moreover, the matrices $\mathscr{A}$ and $\mathscr{L}$ are related to the matrices $\hat{L}$ and $\hat{A}$ from Section 2.3 via (3.18) and (3.19), resp. From this and (3.24) it follows once more that $\tilde{q}(\tilde{\hat{P}})$ equals $q(\hat{P})$ for any $\tilde{\hat{P}} \in N_{g}$. In particular, from $\sum \tilde{q}_{j}=\sum \hat{q}_{j}$ and (2.123) we may infer

$$
\tilde{q}(1)_{j}=\frac{\pi}{N|\mu|}(N+1-2 j), \quad j=1, \ldots, N
$$

At the expense of shrinking $N_{g}$, we may now analytically continue the above functions to any $g \in(-2 \pi / N|\beta \mu|, 0)$. Fixing $g$ in this interval and a correspon$\operatorname{ding} N_{g}$, it is obvious from (3.32)-(3.34) that $\mathscr{A}$ and $\mathscr{L}$ have a one-valued continuation from $N_{g}$ to all of the convex set $\tilde{\Omega}$, and that the key relations (3.18), (3.19) continue to hold.

However, this can no longer be concluded for the functions $\mathscr{B}$ and $B$. Indeed, in the present case we have $\tau>0$, so the argument of the second logarithm in the continuation of (3.37) can now vanish on $\tilde{\hat{\Omega}}$. In view of (3.19) this can only happen for points in the subvariety

$$
\tilde{\Omega}_{b} \equiv\left(\pi\left(\hat{E}_{N}\right) \circ \hat{\mathscr{C}}\right)^{-1}\left(\hat{\Omega}_{b}^{c}\right)
$$

But then the open dense submanifold

$$
\tilde{\Omega}_{r} \equiv \tilde{\hat{\Omega}} \backslash \tilde{\Omega}_{b}
$$

need not be connected, a priori.

Consequently, we restrict attention to the connected component $\tilde{\Omega}_{r 0}$ containing the endpoint $\left(\hat{q}^{0}, \hat{\theta}^{0}\right)$ of $\Gamma$. It follows from previous arguments that $\mathscr{B}$ and $\tilde{q}$ have a one-valued continuation to $\tilde{\hat{\Omega}}_{r 0}\left(\tilde{\boldsymbol{q}}(\tilde{\hat{P}})\right.$ being equal to $q(\hat{\boldsymbol{P}})$ on all of $\left.\tilde{\hat{\Omega}}_{r 0}\right)$, and that (3.25) is valid. However, though $\tilde{\theta}$ can be continued to $\tilde{\Omega}_{r 0}$, the resulting function is not necessarily one-valued, since $\tilde{\Omega}_{r 0}$ need not be simply-connected. (As a matter of fact, $\tilde{\theta}$ is multi-valued and (hence) $\tilde{\hat{\Omega}}_{r 0}$ multiply-connected, cf. Section 4.3.) 
On the other hand, from Section 2.3 we may infer

$$
\left(V^{*} \hat{L} V\right)_{j j}=L_{j j}=\exp \left(\beta\left[\gamma_{j}-\gamma_{j-1}+\gamma_{s}\right]\right) V_{j}^{b}\left(q\left(\delta_{s}, \delta\right)\right)
$$

cf. Lemma 2.3 and (2.82). Let us compare this with (3.52), recalling $q=\tilde{q}$, (1.30) and (3.18), and noting $\sum \tilde{\theta}_{j}=\Sigma \hat{\theta}_{j}=N \hat{\delta}_{s}=N \gamma_{s}$. Then we readily deduce that the diagram (1.85) is well defined and commutative, provided $\tilde{\Omega}_{r}$ is replaced by $\tilde{\Omega}_{r 0}$ and $R$ is defined as the continuation of $B$ to $\tilde{\Omega}_{r 0}$. Moreover, $R$ still inherits the canonicity property from $B_{\mathrm{I}}$. Hence $D R \in S p(2 N, \mathbb{R})$ and so $|D R|=1$. But then the space

$$
\tilde{\Omega}_{r 0} \equiv R\left(\tilde{\Omega}_{r 0}\right)
$$

is an open subset of $\tilde{\Omega}_{r}$. By virtue of (3.48) and commutativity, the image of this set under $\pi\left(E_{N}\right) \circ \mathscr{C}$ equals $\Omega_{r 0}^{c}$, so $\Omega_{r 0}^{c}$ is open. Therefore, the last assertion of the theorem now follows from the commutative diagram obtained from (1.85) upon replacing the four subscripts $r$ by $r 0$.

Next, we prove (3.49). As we have already seen (recall (2.123)), its lhs can be written

$$
\tilde{\mathscr{E}}\left(\hat{\boldsymbol{P}}_{0}(0,0)\right)=\left(0,0 ; \frac{\pi}{N|\mu|}(1, \ldots, 1), \frac{u}{|\beta|}\right)
$$

Thus we should show $u=u_{0}$, where

$$
u_{0} \equiv \frac{\pi}{N}(n, \ldots, j(N-j), \ldots, n)
$$

To this end, consider the symmetric functions of $L\left(0,0 ; \pi(N|\mu|)^{-1}(1, \ldots\right.$, 1), $\gamma$ ). They can be written

$$
S_{l}(x, g)=\sum_{|I|=l}\left(\prod_{J \in I} x_{j}\right) \prod_{\substack{f \in I \\ k \notin I}}\left(1-\frac{\sin ^{2}|\beta \mu| g / 2}{\sin ^{2} \pi(j-k) / N}\right)^{1 / 2}
$$

where $x_{j} \equiv \exp \left(\beta \theta_{j}\right)$ and $\theta$ is defined via (1.30). On the other hand, (3.58) and (2.116) imply

$$
\phi_{r}^{0}\left(\frac{\pi}{N|\mu|}(1, \ldots, 1), \frac{u}{|\beta|}\right)=\left(\frac{\pi}{N|\beta|}(1, \ldots, 1), \frac{u_{0}}{|\mu|}\right)
$$

so the spectrum of $L$ in the point (3.58) consists of the $N$ th roots of $(-1)^{n}$. As a result, this point yields $S_{l}=0, \quad l=1, \ldots, n$, and $S_{N}=1$. 
Now we invoke Lemma A.4 to infer that the system of $N$ equations

$$
S_{l}(x, g)=0, \quad l=1, \ldots, n, \quad S_{N}(x, g)=1
$$

for the unknown vector $x \equiv\left(x_{1}, \ldots, x_{N}\right)$ is solved by the $N$ vectors

$$
x_{j}^{(\nu)} \equiv \exp [i \pi(N+1+2 \nu-2 j) / N], \quad j=1, \ldots, N, \quad \nu=0, \ldots, n
$$

and their complex conjugates. For $N=2,3$ these are readily seen to be the only solutions. However, for $N>3$ one has $N !>2 N$, whereas the system obviously has exactly $N$ ! solutions for $g=0$. By virtue of the implicit function theorem, there exists a unique solution in a $\mathbf{C}^{N}$-neighborhood of each of these for $g \in(-\varepsilon, 0]$, provided $\varepsilon>0$ is sufficiently small. (Indeed, the relevant determinant is non-zero, since it amounts to a non-zero multiple of a Vandermonde determinant for the $N$ th roots of $(-1)^{n}$.) Of course, the above solutions $x^{(\nu)}, \overline{x^{(\nu)}}$ do not depend on $g$; most likely, the remaining solutions do.

Next, consider $L$ evaluated in the point (3.58). In view of (2.116) the corresponding $\hat{L}$ reads

$$
\hat{L}=\operatorname{diag}\left(x_{1}^{(0)}, \ldots, x_{N}^{(0)}\right)
$$

Now $\mathscr{B}\left(\hat{q}^{0}, \hat{\theta}^{0}\right) \rightarrow \mathbf{1}_{N}$ as $g \uparrow 0$, so by (3.25) we have

$$
\left(\hat{f}_{j}^{-1} V_{j k} f_{k}\right)\left(\hat{P}_{0}(0,0)\right) \rightarrow 0, \quad j \neq k, \quad g \uparrow 0
$$

But $\left|\hat{f}_{j}\right|$ and $\left|f_{k}\right|$ do not depend on $g$, cf. (3.58). Hence, the off-diagonal elements of $V\left(\hat{\boldsymbol{P}}_{0}(0,0)\right)$ go to 0 . Combining this with (3.56) and (3.64), we conclude $u \rightarrow u_{0}$ as $g \uparrow 0$. Thus we must have $u=u_{0}$ for sufficiently small $g$, and real-analyticity in $g$ entails $u=u_{0}$ for any $g \in(-2 \pi / N|\beta \mu|, 0)$. Therefore, the proof of (3.49) is now complete.

Combining (3.48) and (3.49), we deduce that $\Omega_{r 0}^{c}$ contains $P_{0}(0,0)$. Furthermore, we have already shown that $\Omega_{r 0}^{c}$ is symplectically diffeomorphic to $\hat{\Omega}_{r 0}^{c}$. Recalling the self-duality property (2.120), a moment's thought suffices to conclude that the second assertion of the theorem holds true.

As announced in Section 1.3, we relegate a consideration of the diagram (1.86) and its consequences to Section 4.3. Note in this connection that the set $\left\{\boldsymbol{P}_{0}\left(\delta_{s}, \gamma_{s}\right) \mid \delta_{s}, \gamma_{s} \in \mathbf{R}\right\} \subset \Omega_{r 0}^{c}$ is not left invariant by the generator (2.39). Thus, at this stage we do not even know whether $\Omega_{r 0}^{c}$ is left invariant by $G$. 


\section{The Harmonic Oscillator Transform}

\subsection{The Case $\mathrm{III}_{\mathrm{nr}}$}

In keeping with our summary in Section 1.2, we begin this section by showing that the map $\phi_{r}$ admits an extension to a $C^{\omega}$ symplectomorphism, cf. (1.71) (1.73). In the process, we shall arrive at a complete picture of the limiting behavior of the invariant tori as one or more action differences $\hat{\delta}_{j}$ converge to their minimum $d / 2$.

It is instructive to detail the case $n=1$ first. Then $\phi_{r}$ can be explicitly determined by exploiting equality of $\operatorname{Tr} \hat{A}(\hat{P}), \operatorname{Tr} \hat{L}(\hat{P})$ and $\operatorname{Tr} A(P), \operatorname{Tr} L(P)$, resp., where $\hat{P} \equiv \tilde{\Phi}(\boldsymbol{P})=\left(\boldsymbol{P}_{2} \times \phi_{r}\right)(\boldsymbol{P})$, cf. (1.66). Indeed, it is straightforward to verify that $\phi_{r}$ is given by

$$
\begin{gathered}
u=-2|\mu|^{-1 / 2} \hat{\delta}(\hat{\delta}+d / 2)^{-1 / 2} \cos |\mu| \delta \\
v=2|\mu|^{-1 / 2}(\hat{\delta}+d / 2)^{-1 / 2} \gamma \sin |\mu| \delta \\
\hat{\delta}=\left(\gamma^{2}+d^{2} / 4 \sin ^{2}|\mu| \delta\right)^{1 / 2}
\end{gathered}
$$

in terms of the coordinates $u, v$ on $\hat{M}^{0}$ and $\delta=\left(q_{1}-q_{2}\right) / 2, \gamma=\left(\theta_{1}-\theta_{2}\right) / 2$ on $M$ (cf. (1.73) and (1.29), (1.30), resp.). Its inverse $b^{0}$ can now be calculated and reads

$$
\begin{gathered}
\delta=\frac{1}{2 \mu} \operatorname{Ln}\left(\frac{u r-i}{u r+i}\right), \quad \operatorname{Ln}(-1) \equiv i \pi \\
r=\frac{1}{2} v r\left(\frac{|\mu|}{2}\left(u^{2}+v^{2}\right)+d\right), \quad d \equiv|\mu g| \\
r \equiv\left(\frac{4|g|+u^{2}+v^{2}}{4 g^{2}+4|g| v^{2}+v^{2}\left(u^{2}+v^{2}\right)}\right)^{1 / 2}
\end{gathered}
$$

Clearly, the boundary set

$$
M_{b} \equiv M \backslash M_{r}
$$

is given by

$$
M_{b}=\{(\pi / 2|\mu|, 0)\}
$$

Moreover, from the above one reads off that $\phi_{r}$ and $\mathrm{b}^{0}$ extend to $C^{\omega}$ maps $\phi: M$ $\rightarrow \hat{M}$ and $b: \hat{M} \rightarrow M$, as advertised. The state of affairs is depicted in Figure 1 . 


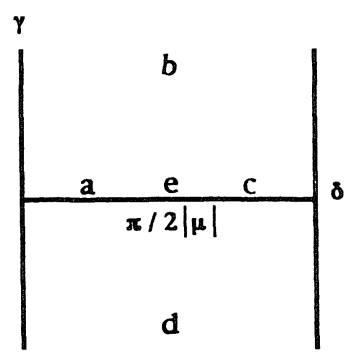

M

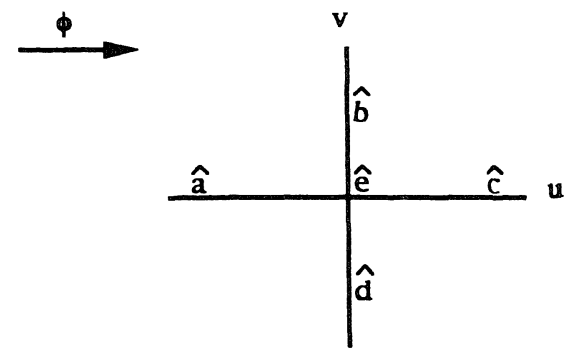

$\hat{\mathbf{M}}$

Figure 1. The $n=1$ maps $\phi, \mathrm{b}$ and spaces $\hat{M}^{f}$. The indicated points are related by $\hat{p}=\phi(p)$.

It appears out of the question to calculate $b^{0}$ and $\phi_{r}$ explicitly for $n>1$. Of course, the cases $n=2,3$ should still be accessible via Cardano's formulas, but it seems unlikely that this would yield substantially more information than the following theorem, which handles the general $n$ case.

Theorem 4.1. The maps $\phi_{r}$ and $\mathrm{b}^{0}$ extend to mutually inverse $C^{\omega}$ symplectomorphisms $\phi$ and $\mathrm{b}$ between $\langle\boldsymbol{M}, \omega(\boldsymbol{M})\rangle$ and $\langle\hat{M}, \omega(\hat{M})\rangle$, where the symplectic forms are given by (1.33) and (1.72), resp. The boundary set (4.7) has codimension 2 and one has

$$
\mathrm{b}(0,0)=\left(\frac{\pi}{N|\mu|}(1, \ldots, 1), 0\right)
$$

Proof. Since we restrict attention to the internal spaces $M$ and $\hat{M}$, we may and will take $\hat{\delta}_{s}^{f}, \hat{\gamma}_{s}^{f}=0$ in the above and replace the set $\{(0,0)\} \times \hat{M}^{f}$ by $\hat{M}^{f}$. First, we note $L$ is $C^{\omega}$ on $M$ and we recall $\sigma(L)$ is real and simple on $M$. Thus the eigenvalues of $L$ are $C^{\omega}$ on $M$. As a consequence, the vector $\hat{\theta} \in A_{N}$ has a $C^{\omega}$ extension from $M_{r}$ to all of $M$, and the extension satisfies $\hat{\theta} \in A_{N}^{c l}$, where $c l$ denotes closure.

Next, we note that the lhs of (2.18) and (2.19) can be rewritten

$$
\begin{aligned}
\left(U^{*} A U\right)_{k, k+1} & =\tilde{C}(0, \mu,-g ; \hat{\theta})_{k, k+1} \\
& =\frac{d}{\left(\hat{\theta}_{k+1}-\hat{\theta}_{k}\right)} \prod_{l \neq k, k+1}\left(\left(1-\frac{d}{\hat{\theta}_{k}-\hat{\theta}_{l}}\right)\left(1+\frac{d}{\hat{\theta}_{k+1}-\hat{\theta}_{l}}\right)\right)^{1 / 2} \\
\left(U^{t} e\right)_{1} & =\hat{g}_{1}=\prod_{l>1}\left(1+\frac{d}{\hat{\theta}_{1}-\hat{\theta}_{l}}\right)^{1 / 2}
\end{aligned}
$$

From these formulas one reads off that the rhs has a non-zero $C^{\omega}$ extension to all 
of $M$. (Note, in particular, that the rhs of (4.10) converges to -1 as $\hat{\theta}_{k}-\hat{\theta}_{k+1} \downarrow$ d.) Therefore, we may and will define a unitary $U$ satisfying

$$
U^{*} L U=\operatorname{diag}\left(\hat{\theta}_{1}, \ldots, \hat{\theta}_{N}\right), \quad \hat{\theta} \in A_{N}^{c l}
$$

and (2.18), (2.19) on all of $M$. (Thus, this unitary $U$ coincides on $M_{r}$ with the unitary $U$ from Section 2.1.)

Now we fix $m=(\delta, \gamma) \in M$ and a ball $B^{c} \subset \mathbf{C}^{2 n}$ with center $m$ such that $L$ is holomorphic and $\sigma(L)$ simple in $B^{c}$. Eventually shrinking the radius, there exists a holomorphic map $\mathscr{D}: B^{c} \rightarrow G L(N, \mathrm{C})$ such that

$$
\mathscr{D}^{-1} L \mathscr{D}=\operatorname{diag}\left(\lambda_{1}, \ldots, \lambda_{N}\right), \quad \lambda_{N}(m)<\cdots<\lambda_{1}(m)
$$

on $B^{c}$, and we may ensure $B \equiv B^{c} \cap \mathbf{R}^{2 n}$ is a ball in $M$. (In particular, the radius should be smaller than $\pi /|\mu|$.) Then the restriction of $\mathscr{D}$ to $B$ is $C^{\omega}$ in $B$. Since the eigenvalue ordering on $B$ is fixed by the order in $m$, and since the order imposed in $m$ coincides with the order corresponding to $U$, it follows that on $B$ one has

$$
\mathscr{D}=U \operatorname{diag}\left(\chi_{1}, \ldots, \chi_{N}\right), \quad \chi_{j}: B \rightarrow \mathbf{C}^{*}
$$

We now claim that the quantities

$$
\phi_{k} \equiv \chi_{k} / \chi_{k+1}, \quad k=1, \ldots, n
$$

are $C^{\omega}$ in $B$. Indeed, from (4.14) we have

$$
\phi_{k}\left(\mathscr{D}^{-1} A \mathscr{D}\right)_{k, k+1}=\left(U^{*} A U\right)_{k, k+1}, \quad k=1, \ldots, n
$$

Since the rhs and $\phi_{k}$ are non-zero on $B$, the matrix element at the lhs is non-zero. Moreover, this element and the rhs are $C^{\omega}$ in $B$, so the claim follows. As a consequence, the renormalized matrix

$$
\mathscr{D}_{r} \equiv \mathscr{D} \operatorname{diag}\left(1, \phi_{1}, \phi_{1} \phi_{2}, \ldots, \phi_{1} \cdots \phi_{n}\right)
$$

is $C^{\omega}$ in $B$ and satisfies

$$
\mathscr{D}_{r}=\chi_{1} U
$$

Now consider the equality

$$
\left(\mathscr{D}_{r}^{t} e\right)_{1}=\chi_{1}\left(U^{t} e\right)_{1}
$$


Since $\left(U^{t} e\right)_{1}$ is non-zero and $C^{\omega}$ in $M$ and the lhs is $C^{\omega}$ in $B$, we may infer that $\chi_{1}$ is $C^{\omega}$ in $B$. In view of (4.18), we may then conclude that the map $M \rightarrow U(N),(\delta$, $\gamma) \longmapsto U$ is $C^{\omega}$.

We have now shown that $\hat{\delta}$ and $U$ have $C^{\omega}$ extensions to $M$. Next, we exploit this to prove that $\phi_{r}$ has a $C^{\omega}$ extension to $M$, provided $\hat{M}^{0}$ is viewed as being densely embedded in $\hat{M}$ via (1.73). To this end we introduce $(u, v) \in \mathbf{R}^{2 n}$ by setting

$$
u_{j}+i v_{j} \equiv\left(U^{t} e\right)_{j^{+1}} / N_{j}, \quad j=1, \ldots, n
$$

where

$$
N_{j} \equiv-\frac{1}{2}\left(\frac{|\mu|}{\hat{\delta}_{j}}\right)^{1 / 2} \prod_{k \neq \neq, j+1}\left(1+\frac{d}{\hat{\theta}_{j+1}-\hat{\theta}_{k}}\right)^{1 / 2}
$$

Since $N_{j}$ is negative on all of $M$, and $U, e$ and $N_{j}$ are $C^{\omega}$ on $M$, it follows that $u$ and $v$ are well-defined $C^{\omega}$ functions on $M$. Furthermore, comparing (4.20), (4.21) and (2.8), (2.22), we deduce that on $M_{r}$ the relation between $u, v$ and $\hat{\delta}, \hat{\gamma}$ is given by (1.73). Therefore, $\phi_{r}$ extends to a $C^{\omega}$ map

$$
\phi: M=\mathbf{w}_{n} \times \mathbf{R}^{n} \longrightarrow \hat{M}=\mathbf{R}^{2 n}, \quad(\delta, \gamma) \longmapsto(u, v)
$$

as announced.

To prove that $\mathrm{b}^{0}$ admits a $C^{\omega}$ extension to $\hat{M}$ after the coordinate change (1.73) on $\hat{M}^{0}$, we begin by studying the vectors $(2.22),(2.23)$ as functions of the variables $u, v$. Recalling (2.8), (4.11), (4.20) and (4.21), we infer that $\hat{g}$ has a $C^{\omega}$ extension to $\hat{M}$. Also, $\hat{f}$ may be written (recall $\hat{\gamma}_{s} \equiv 0$ )

$$
\begin{gathered}
\hat{f}_{j}=\frac{1}{2}\left(-u_{j}+i v_{j}\right)\left(\frac{|\mu|}{\hat{\delta}_{j}}\right)^{1 / 2} \prod_{k \neq j, j+1}\left(1-\frac{d}{\hat{\theta}_{j}-\hat{\theta}_{k}}\right)^{1 / 2}, j=1, \ldots, n \\
\hat{f}_{N}=\prod_{k<N}\left(1-\frac{d}{\hat{\theta}_{N}-\hat{\theta}_{k}}\right)^{1 / 2}
\end{gathered}
$$

from which we read off that $\hat{f}$ has a $C^{\omega}$ extension to $\hat{M}$, too. Clearly, the same is true for $C(0, \mu,-g ; \hat{\theta})_{k l}$, provided $l \neq k+1$. Therefore, $\hat{A}_{k l}$ has a $C^{\omega}$ extension for $l \neq$ $k+1$, cf. (2.11). But $\hat{A}_{k, k+1}$ is given by the rhs of (4.10), and from this equation one sees that these elements have $C^{\omega}$ extensions as well.

The upshot is, that $\hat{f}, \hat{g}$ and $\hat{A}$ have $C^{\omega}$ extensions to $\hat{M}$. Since the matrix $\hat{A}$ is unitary on $\hat{M}^{0}$ and continuous on $\hat{M}$, it is unitary on $\hat{M}$. Likewise, the commutation relation (2.26) holds true on $\hat{M}$. Thus we may extend the diagonalizing unitary $V$ to $\hat{M}$ by repeating the steps that defined $V$ on $\hat{\Omega}^{c}$, cf. (2.25)-(2.36), (2.41). (Note 
in this connection that for $q \in W_{N}$ the constraint $\Sigma q_{j}=0$ does not entail $q \in F_{N}$ when $N>2$.) Along the way, we infer in the same way as before that $\sigma(\hat{A})$ is actually simple on all of $\hat{M}$. Since $\hat{A}$ is also $C^{\omega}$ on $\hat{M}$, we may deduce that the eigenvalues of $\hat{A}$ are $C^{\omega}$ on $\hat{M}$. But then it follows that the $C^{\omega}$ map $\hat{M}^{0} \rightarrow \mathrm{w}_{n},(u$, $v) \longmapsto \delta$ has a $C^{\omega}$ extension to $\hat{M}$, mapping $\hat{M}$ into $\mathrm{w}_{n}$. (Note that $A(q)$ has degenerate spectrum on the walls of the alcove $w_{n}$ )

To prove that $\gamma$ has a $C^{\omega}$ extension, too, we choose $\hat{m} \in \hat{M}$, a ball $\hat{B} \subset \hat{M}$ around $\hat{m}$, and a $C^{\omega}$ map $\hat{\mathscr{D}}: \hat{B} \rightarrow G L(N, \mathbb{C})$ such that

$$
\left(\hat{\mathscr{D}}^{-1} \hat{A} \hat{\mathscr{D}}\right)_{j k}=0, j \neq k, \quad\left(\hat{\mathscr{D}}^{-1} \hat{A} \hat{\mathscr{D}}\right)(\hat{m})_{j j}=\left(V^{*} \hat{A} V\right)(\hat{m})_{j j}, j, k=1, \ldots, N
$$

Now the matrix $V^{*} \hat{A} V$ equals $A(q)$ and so is $C^{\omega}$ on $\hat{M}$. Hence, it equals $\hat{\mathscr{D}}^{-1} \hat{A} \hat{\mathscr{D}}$ on $\hat{B}$ due to (4.25). Therefore, we must have

$$
\hat{\mathscr{D}}=V \operatorname{diag}\left(\hat{\chi}_{1}, \ldots, \hat{\chi}_{N}\right), \hat{\chi}_{j}: \hat{B} \longrightarrow \mathbb{C}^{*}
$$

which entails

$$
\left(\hat{\mathscr{D}}^{-1} \hat{L} \hat{\mathscr{D}}\right)_{j j}=\left(V^{*} \hat{L} V\right)_{j j}, \quad j=1, \ldots, N
$$

As a consequence, the lhs of (4.27) is a real-valued function on $\hat{B}$, which we denote by $\theta_{j}$, in agreement with (2.37) for points in $\hat{\boldsymbol{B}} \cap \hat{\boldsymbol{M}}^{0}$. Defining $\gamma$ via (1.30) (with $\gamma_{s}=0$, of course), it follows that $\gamma$ is $C^{\omega}$ in $\hat{B}$.

The upshot is, that $b^{0}$ has a $C^{\omega}$ extension $b$, as announced. The relations

$$
\mathrm{b}^{0} \circ \phi_{r}=\mathrm{id}\left(M_{r}\right), \quad \phi_{r} \circ \mathrm{b}^{0}=\mathrm{id}\left(\hat{M}^{0}\right)
$$

then extend by continuity to

$$
\mathrm{b} \circ \phi=\mathrm{id}(M), \quad \phi \circ \mathrm{b}=\mathrm{id}(\hat{M})
$$

so that $\mathrm{b}$ and $\phi$ are mutually inverse bijections. Moreover, since $\mathrm{b}$ and $\phi$ are symplectic on dense submanifolds by virtue of Theorem 3.1, the first assertion of the theorem now follows.

Since $\hat{M} \backslash \hat{M}^{0}$ is the subvariety of $\hat{M}=\mathbb{R}^{2 n}$ for which at least one $\left(u_{j}, v_{j}\right)$ equals $(0,0)$, it has codimension 2. Hence its image $M_{b}$ under $b$ also has codimension 2 .

Finally, to prove (4.9) we observe that $b(0,0)$ is the point in $M$ that is uniquely determined by all eigenvalue distances $\hat{\theta}_{j}-\hat{\theta}_{j+1}, j=1, \ldots, n$, being equal to $d$. Now the point at the rhs of (4.9) corresponds to the point $\left(q^{e}, \theta^{e}\right) \in \tilde{\Omega}$ given by 


$$
q_{j}^{e}=\frac{\pi}{N|\mu|}(N+1-2 j), \quad \theta_{j}^{e}=0, \quad j=1, \ldots, N
$$

The Lax matrix (2.1) evaluated in this point equals $i d E_{1} / 2$, where $E_{1}$ is given by (A.31). Hence its eigenvalues read

$$
\hat{\theta}_{j}=\frac{d}{2}(N+1-2 j), \quad j=1, \ldots, N
$$

by virtue of (A.33). Thus, (4.9) follows.

Now that we have the relevant maps and their key properties under control, a comment on the strategy we have followed is in order. From (3.19) and (3.25) one sees that the matrix-valued functions $\mathscr{L}$ and $\mathscr{B}$ do not admit a regular extension from $\hat{M}^{0}$ to $\hat{M}$. (To check this, recall $\hat{f}$ is given by (2.23).) However, by using $\mathscr{L}$ and $\mathscr{B}$ we could solve the canonicity problem via the results of $\mathrm{I}$ and analytic continuation. Just as in II and in the cases $\mathrm{III}_{\text {rel }}$ and $\mathrm{II}_{\mathrm{b}}$, the gauge fixing $\mathscr{B} \zeta=\zeta$ involving the constant vector $\zeta=(1, \ldots, 1)$ (as opposed to functions with singularities) enabled us to control the continuation between the different parameter regimes. But we employed $\hat{A}$ and $V$ to handle the extension of the action-angle transform to the harmonic oscillator transform, since these functions admit a $C^{\omega}$ extension from $\hat{M}^{0}$ to $\hat{M}$.

To complete this section we detail the results for $\tilde{\Omega}$ and $\Omega$ following from Theorem 4.1.

Corollary 4.2. The maps $\tilde{\Phi}$ and $\tilde{\mathscr{E}}$ extend to mutually inverse $C^{\omega}$ symplectomorphisms $\tilde{\Phi}^{\#}=P_{2} \times \phi$ and $\tilde{\mathscr{E}} \#=P_{2} \times \mathrm{b}$ between $\langle\tilde{\Omega}, \tilde{\omega}\rangle$ and $\left\langle\hat{\Omega}^{\# c}, \hat{\omega}^{\# c}\right\rangle$, where

$$
\hat{\Omega}^{\# c} \equiv \mathbf{R}^{2} \times \hat{M}, \quad \hat{\omega}^{\# c} \equiv N d \hat{\gamma}_{s} \wedge d \hat{\delta}_{s}+\sum_{j=1}^{n} d u_{j} \wedge d v_{j}
$$

The boundary set (3.26) has codimension 2 and one has

$$
\tilde{\mathscr{E}}^{\#}(0,0 ; 0,0)=\left(q^{e}, \theta^{e}\right)
$$

where the rhs is given by (4.30).

Proof. This is clear from Theorems 3.1 and 4.1 .

Now we transform the free symplectic $\mathbf{Z}$-action on $\tilde{\Omega}$ generated by $G$ (recall (1.12)) into a free symplectic $\hat{\mathbf{Z}}$-action on $\hat{\Omega}^{\# c}$ via $\tilde{\Phi}^{\#}$. Thus the latter action extends the $\hat{\mathbf{Z}}$-action generated by $\hat{G}$ on $\hat{\Omega}^{c}$ (recall (2.40)). Next, we set

$$
\hat{\Omega}^{\#} \equiv \hat{\Omega}^{\# c} / \hat{\mathbf{Z}}, \quad \hat{\omega}^{\#} \equiv \hat{\omega}^{\# c} / \hat{\mathbf{Z}}
$$


and define a map $\Phi^{\#}$ by requiring that the diagram (1.74) commute. $\left.\hat{\omega}^{\#}\right\rangle$.

Corollary 4.3. The map $\Phi^{\#}$ is a symplectomorphism from $\langle\Omega, \omega\rangle$ onto $\left\langle\hat{\Omega}^{\#}\right.$,

Proof. Obvious from the above.

We finish this section by supplying an illuminating coordinatization for $\hat{\Omega}^{\#}$. Namely, we set

$$
\hat{\Omega}^{\#} \simeq\left\{(\hat{x}, \hat{p} ; a, b) \in \mathbf{R}^{2 N} \mid \hat{x} \in[0,2 \pi / N|\mu|)\right\}
$$

where the relation to the coordinates $\left(\hat{\delta}_{s}, \hat{\gamma}_{s} ; u, v\right)$ on $\hat{\Omega}^{\# c}$ is defined by

$$
\begin{aligned}
& \exp (N \mu \hat{x}) \equiv \exp \left(N \mu \hat{\gamma}_{s}\right), \quad \hat{p} \equiv N \hat{\delta}_{s} \\
& \exp \left[\mu\left(\hat{\gamma}_{s}-\hat{x}\right)\right]=\exp (2 \pi i l / N) \Longrightarrow \\
&\left(a_{j}, b_{j}\right)^{t} \equiv R(2 \pi j l / N)\left(u_{j}, v_{j}\right)^{t}, j=1, \ldots, n \\
& R(\phi) \equiv\left(\begin{array}{rr}
\cos \phi & \sin \phi \\
-\sin \psi & \cos \phi
\end{array}\right)
\end{aligned}
$$

(Note that $l$ takes values $0,1, \ldots, n$, depending on $\hat{\gamma}_{s}$ ) Using $(2.40)$ it is easy to verify that this makes sense. (That is, the coordinates are in 1-1 correspondence to $\hat{\mathbf{Z}}$-orbits in $\hat{\Omega}^{\# c}$.) Also, on the open dense patch $\{\hat{x} \neq 0\}$ one has

$$
\hat{\omega}^{\#}=d \hat{x} \wedge d \hat{p}+\sum_{j=1}^{N-1} d a_{j} \wedge d b_{j}
$$

Finally, we point out that the action-angle phase space $\hat{\Omega}$ equals the submanifold of $\hat{\Omega}^{\#}$ given by $\left(a_{j}, b_{j}\right) \neq(0,0), j=1, \ldots, n$, and that the relation to the coordinates $\left(\hat{x}_{1}, \ldots, \hat{p}_{N}\right)$ on $\hat{\Omega}$ is given by

$$
\begin{gathered}
\hat{p}=\hat{p}_{1}+\cdots+\hat{p}_{N}, \quad a_{j}^{2}+b_{j}^{2}=\frac{2}{|\mu|}\left(\hat{p}_{j}-\hat{p}_{j+1}-d\right), j=1, \ldots, n \\
e^{\mu \hat{x}} \operatorname{diag}\left(\phi_{1}, \phi_{2} \overline{\phi_{1}}, \phi_{3} \overline{\phi_{2}}, \ldots, \overline{\phi_{n}}\right)=\operatorname{diag}\left(e^{\mu \hat{x}_{1}}, \ldots, e^{\mu \hat{x}_{N}}\right)
\end{gathered}
$$

with

$$
\phi_{j} \equiv\left(-a_{j}+i b_{j}\right) /\left(a_{j}^{2}+b_{j}^{2}\right)^{1 / 2}, \quad j=1, \ldots, n
$$

Observe that (4.41) amounts to a picture of the obvious maximal $U(N)$-torus as a fiber bundle over $U(1)$ (given by the determinant) with fiber the obvious maximal 
$S U(N)$-torus and transition function $\exp (2 \pi i / N) \mathbf{1}_{N}$.

\subsection{The Case $\mathrm{III}_{\text {rel }}$}

Just as in previous chapters, this case can be treated along the same lines as the $\mathrm{III}_{\mathrm{nr}}$ case. Thus, we start again by detailing the $n=1$ situation. Proceeding as before, we find that $\phi_{r}$ reads

$$
\begin{aligned}
& u=-2|\mu|^{-1 / 2} \operatorname{sh} \beta \hat{\delta}\left(\frac{\hat{\delta}-d / 2}{\operatorname{sh}^{2} \beta \hat{\delta}-\operatorname{sh}^{2} z}\right)^{1 / 2} \cos |\mu| \delta \\
& v=2|\mu|^{-1 / 2} \operatorname{ch} \beta \hat{\delta}\left(\frac{\hat{\delta}-d / 2}{\operatorname{sh}^{2} \beta \hat{\delta}-\operatorname{sh}^{2} z}\right)^{1 / 2} \operatorname{th} \beta \gamma \sin |\mu| \delta
\end{aligned}
$$

where

$$
\hat{\delta}=\frac{1}{\beta} \operatorname{Arth}\left(\left[\operatorname{th}^{2} \beta \gamma \sin ^{2}|\mu| \delta+\operatorname{sh}^{2} z\right]^{1 / 2} /\left[\sin ^{2}|\mu| \delta+\operatorname{sh}^{2} z\right]^{1 / 2}\right)
$$

Also, the $\delta$-part of $b^{0}$ is again given by (4.4) and $\gamma$ reads

$$
\gamma=\frac{1}{\beta} \operatorname{Arth}\left(v r t h \frac{\beta}{2}\left(\frac{|\mu|}{2}\left[u^{2}+v^{2}\right]+d\right)\right)
$$

where we now have

$$
r \equiv\left(\frac{\operatorname{sh}^{2} \frac{\beta}{2}\left(\frac{|\mu|}{2}\left[u^{2}+v^{2}\right]+d\right)-\operatorname{sh}^{2} z}{u^{2} \operatorname{sh}^{2} z+v^{2} \operatorname{sh}^{2} \frac{\beta}{2}\left(\frac{|\mu|}{2}\left[u^{2}+v^{2}\right]+d\right)}\right)^{1 / 2}, \quad z \equiv \beta d / 2
$$

Note these formulas reduce to (4.1)-(4.6) for $\beta \downarrow 0$. The boundary set (4.7) is again given by (4.8), and the real-analytic extension properties are manifest from (4.43)-(4.47). Moreover, Figure 1 applies once more.

Theorem 4.4. The assertions of Theorem 4.1 hold true, with $\phi_{r}$ and $\mathrm{b}^{0}$ denoting maps defined in Section 3.2.

Proof. The proof of Theorem 4.1 can be mimicked to a large extent. First, since $L$ is $C^{\omega}$ on $M$ (cf. (2.51)) and $\sigma(L)$ is positive and simple on $M$, it follows that $\hat{\theta}$ has a $C^{\omega}$ extension to $M$, taking values in $A_{N}^{c l}$. Next, we rewrite the lhs of (2.18) and (2.19) as 


$$
\begin{aligned}
&\left(U^{*} A U\right)_{k, k+1}= \frac{\operatorname{sh} z}{\operatorname{sh} \frac{\beta}{2}\left(\hat{\theta}_{k+1}-\hat{\theta}_{k}\right)} \\
& \cdot \prod_{l \neq k, k+1}\left(\frac{\operatorname{sh} \frac{\beta}{2}\left(\hat{\theta}_{k}-\hat{\theta}_{l}-d\right)}{\operatorname{sh} \frac{\beta}{2}\left(\hat{\theta}_{k}-\hat{\theta}_{l}\right)} \frac{\operatorname{sh} \frac{\beta}{2}\left(\hat{\theta}_{k+1}-\hat{\theta}_{l}+d\right)}{\operatorname{sh} \frac{\beta}{2}\left(\hat{\theta}_{k+1}-\hat{\theta}_{l}\right)}\right)^{1 / 2} \\
&\left(U^{t} e\right)_{1}=e^{-\frac{n}{2} z+\frac{\beta}{2} \hat{\theta}_{1}} \prod_{l>1}\left(\frac{\operatorname{sh} \frac{\beta}{2}\left(\hat{\theta}_{1}-\hat{\theta}_{l}+d\right)}{\operatorname{sh} \frac{\beta}{2}\left(\hat{\theta}_{1}-\hat{\theta}_{l}\right)}\right)^{1 / 2}
\end{aligned}
$$

cf. (2.6) $-(2.8),(2.59)-(2.61)$ and (2.72). From this we see that these quantities have non-zero $C^{\omega}$ extensions to $M$. (The rhs of (4.48) again goes to -1 as $\hat{\theta}_{k}-$ $\hat{\theta}_{k+1} \downarrow d$.) Thus we may uniquely determine a unitary $U$ on $M$ by requiring (2.18), (2.19) and

$$
U^{*} L U=\operatorname{diag}\left(e^{\beta \hat{\theta}_{1}}, \ldots, e^{\beta \hat{\theta}_{N}}\right), \quad \hat{\theta} \in A_{N}^{c l}
$$

Then it follows in the same way as before that $U$ is $C^{\omega}$ on $M$. Moreover, replacing (4.21) by

$$
N_{j} \equiv-\frac{1}{2} e^{-\frac{n}{2} z+\frac{\beta}{2} \hat{\theta}_{J+1}}\left(\frac{|\mu| \operatorname{sh} \beta\left(\hat{\delta}_{j}-d / 2\right)}{\left(\hat{\delta}_{j}-d / 2\right) \operatorname{sh} \beta \hat{\delta}_{j}}\right)^{1 / 2} \prod_{k \neq j, J+1}\left(\frac{\operatorname{sh} \frac{\beta}{2}\left(\hat{\theta}_{j+1}-\hat{\theta}_{k}+d\right)}{\operatorname{sh} \frac{\beta}{2}\left(\hat{\theta}_{j+1}-\hat{\theta}_{k}\right)}\right)^{1 / 2}
$$

and (2.22) by (2.59), the paragraph containing (4.20) applies verbatim. (Recall the positive factor $\xi$ in (2.59) is given by (2.72).)

Using (4.49) and (4.51) we see that $\hat{g}$ has a $C^{\omega}$ extension to $\hat{M}$. Since $\hat{f}$ can be written

$$
\begin{gathered}
\hat{f}_{j}=\frac{1}{2}\left(-u_{j}+i v_{j}\right) e^{\frac{n}{2} z+\frac{\beta}{2} \hat{\theta}_{J}}\left(\frac{|\mu| \operatorname{sh} \beta\left(\hat{\delta}_{j}-d / 2\right)}{\left(\hat{\delta}_{j}-d / 2\right) \operatorname{sh} \beta \hat{\delta}_{j}}\right)^{1 / 2} \prod_{k \neq j, j+1}\left(\frac{\operatorname{sh} \frac{\beta}{2}\left(\hat{\theta}_{j}-\hat{\theta}_{k}-d\right)}{\operatorname{sh} \frac{\beta}{2}\left(\hat{\theta}_{j}-\hat{\theta}_{k}\right)}\right)^{1 / 2} \\
\hat{f}_{N}=e^{\frac{n}{2} z+\frac{\beta}{2} \hat{\theta}_{N}} \prod_{k<N}\left(\frac{\operatorname{sh} \frac{\beta}{2}\left(\hat{\theta}_{N}-\hat{\theta}_{k}-d\right)}{\operatorname{sh} \frac{\beta}{2}\left(\hat{\theta}_{N}-\hat{\theta}_{k}\right)}\right)^{1 / 2}
\end{gathered}
$$


it manifestly has a $C^{\omega}$ extension to $\hat{M}$ as well. From (4.48) we conclude that $\hat{A}_{k, k+1}$ has a $C^{\omega}$ extension, so it follows again that $\hat{A}$ has a $C^{\omega}$ extension to $\hat{M}$.

We are now in the position to extend $V$ to $\hat{M}$ via (the extension of ) (2.62)(2.66), (2.68)-(2.70). (Note that $\xi=\xi^{-1}$.) From (2.63) we deduce as before that $\sigma(\hat{A})$ is simple on $\hat{M}$, so it follows that $\delta$ has a $C^{\omega}$ extension to $\hat{M}$ with values in $\mathbf{w}_{n}$.

The paragraph containing (4.27) now applies verbatim. Since the rhs of (4.27) is positive on $\hat{M}$, the lhs is positive on $\hat{B}$. Thus we may and will introduce $\theta \in \mathbf{R}^{N}$ by setting

$$
\left(\hat{\mathscr{D}}^{-1} \hat{L} \hat{\mathscr{D}}\right)_{j j} \equiv e^{\beta \theta_{J}} V_{j}(q), \quad j=1, \ldots, N
$$

(This agrees with (2.67) for points in $\hat{B} \cap \hat{M}^{0}$.) Defining $\gamma$ by (1.30), we thus obtain a $C^{\omega}$ extension of $\gamma$ to $\hat{B}$. As a consequence $b^{0}$ has a $C^{\omega}$ extension b to $\hat{M}$, so the first assertion of the theorem follows as before via (4.28), (4.29).

Now it is again obvious that codim $M_{b}=2$, so it remains to prove that the vector $\hat{\theta}$ in the point (4.30) is given by (4.31). But since we have

$$
L\left(q^{e}, \theta^{e}\right)=E
$$

with $E$ given by (A.29), this follows from (A.32).

The comment after Theorem 4.1 applies to the present case, too. Note that the explicit formula (2.72) for $\xi$ has not been used in the proof of Theorem 3.3, whereas it is needed in the proof of Theorem 4.4. (A priori, $\xi$ need not be $C^{\omega}$ on M.)

The paragraph containing (4.34) may and will be taken over verbatim.

Corollary 4.5. The assertions of Corollaries 4.2 and 4.3 hold true in the $\mathrm{III}_{\mathrm{rel}}$ case, too.

Proof. This follows from Theorems 3.3 and 4.4.

Clearly, the paragraphs containing (4.35)-(4.42) can also be applied to the case III $_{\text {rel }}$.

\subsection{The Case III $_{b}$}

We begin again by studying the case $n=1$ in considerable detail. This will enable us to obtain a complete picture of the extensions already described in general terms in Section 1.3. In particular, the real-analyticity of the map (1.91) will be explicitly verified. 
Proceeding as before, we find that $\phi_{r}^{0}$ reads

$$
\begin{gathered}
\hat{\delta}=\frac{1}{2 \beta} \operatorname{Ln}\left(\frac{Q \cos |\beta| \gamma+i}{\text { c.c. }}\right), \quad \operatorname{Ln}(-1) \equiv i \pi \\
\hat{\gamma}=\frac{1}{|\mu|} \operatorname{Arg}(\cos |\mu| \delta+i Q \sin |\beta| \gamma \sin |\mu| \delta), \quad \operatorname{Arg} z \in(-\pi, \pi]
\end{gathered}
$$

where

$$
Q \equiv\left(\frac{\sin ^{2}|\mu| \delta-\sin ^{2} \tau}{\sin ^{2} \tau \cos ^{2}|\beta| \gamma+\sin ^{2}|\mu| \delta \sin ^{2}|\beta| \gamma}\right)^{1 / 2}
$$

Then $b_{r}^{0}$ is given by the interchanges $\beta \leftrightarrow \mu, \gamma \leftrightarrow \hat{\gamma}, \delta \leftrightarrow \hat{\delta}$, in agreement with self-duality. Moreover, introducing

$$
\hat{M}_{b}^{f 0} \equiv \hat{M}^{f 0} \backslash \hat{M}_{r}^{f 0}
$$

we obtain

$$
M_{b}^{0}=\left\{\left(\frac{\pi}{2|\mu|}, 0\right),\left(\frac{\pi}{2|\mu|}, \frac{\pi}{|\beta|}\right)\right\}, \quad \hat{M}_{b}^{0}=\left\{\left(\frac{\pi}{2|\beta|}, 0\right),\left(\frac{\pi}{2|\beta|}, \frac{\pi}{|\mu|}\right)\right\}
$$

Evidently, $\hat{M}_{b}^{f 0}$ has codimension 2 and $\hat{M}_{r}^{f 0}$ is connected, but not simply-connected. Figure 2 provides a sketch of the spaces and maps.

As indicated in this picture, when the boundary points $(\pi / 2|\mu|, 0),(\pi / 2|\mu|$, $\pi /|\beta|)$ in $M^{0}$ are approached, $\hat{\delta}$ converges to the endpoints $\tau /|\beta|,(\pi-\tau) /|\beta|$, resp., of its definition interval, whereas $\hat{\gamma}$ has direction-dependent limits, cf. (4.56)-

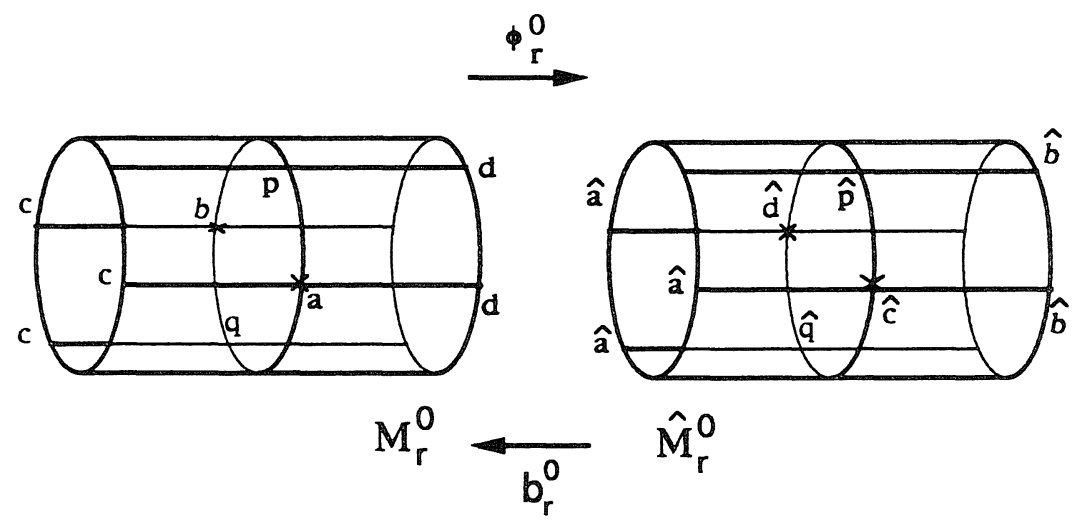

Figure 2. The $n=1$ maps $\phi_{r}^{0}, \mathrm{~b}_{r}^{0}$ and spaces $\hat{M}_{r}^{f 0}$. The crossed points and bounding circles do not belong to $\hat{M}_{r}^{f 0}$. 

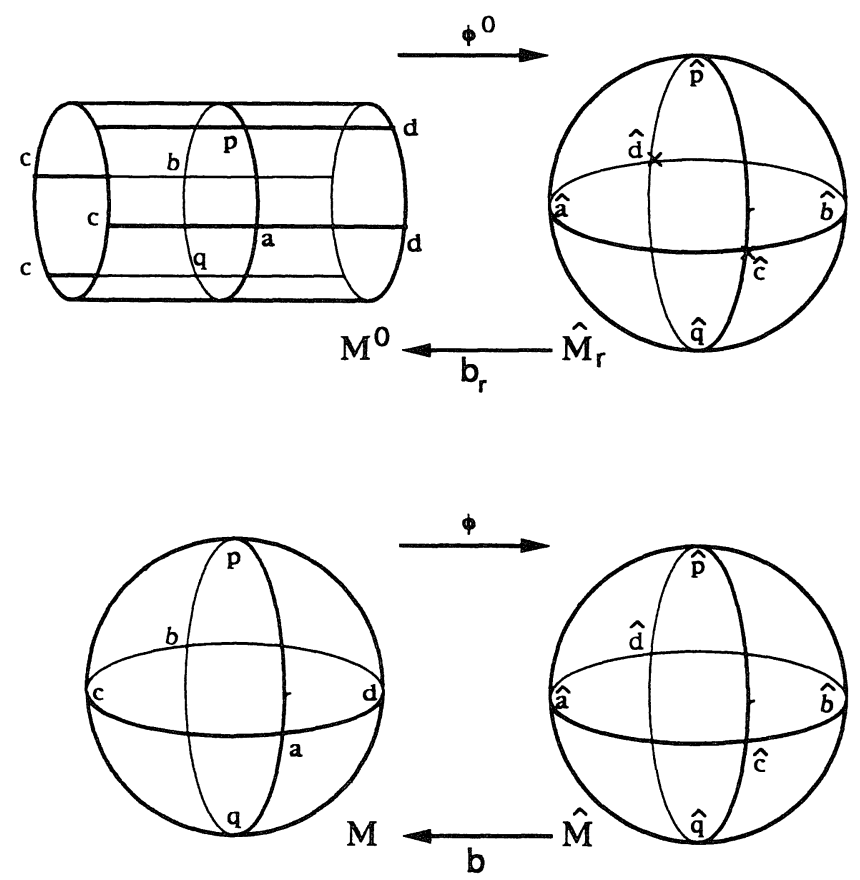

Figure 3. The two-step extension procedure for $n=1$.

(4.58). Thus, we may extend $\hat{M}_{r}^{0}$ with two 'endpoints' by letting the $\hat{\gamma}$-torus collapse to these points as $\hat{\delta} \downarrow \tau /|\beta|$ and $\hat{\delta} \uparrow(\pi-\tau) /|\beta|$, resp. Then $\phi_{r}^{0}$ has a continuous extension $\phi^{0}: M^{0} \rightarrow \hat{M}_{r}$, where $\hat{M}_{r}$ is the extension of $\hat{M}_{r}^{0}$.

Next, we note that when we fix $\gamma$ and take $\delta \downarrow \tau /|\mu|$ and $\delta \uparrow(\pi-\tau) /|\mu|$, we get $(\hat{\delta}, \hat{\gamma}) \rightarrow(\pi / 2|\beta|, 0),(\pi / 2|\beta|, \pi /|\mu|)$, irrespective of the fixed $\gamma$-value, cf. (4.56)-(4.58). Thus, it is clear how the loss of self-duality incurred in the extension can be restored : We should extend $M^{0}$ with two 'endpoints', too, and $\hat{M}_{r}$ with the two boundary points of $\hat{M}^{0}$, yielding (topological) manifolds $M, \hat{M} \simeq S^{2}$ and mutually inverse homeomorphisms $\phi: M \rightarrow \hat{M}, \mathrm{~b}: \hat{M} \rightarrow M$. The situation is depicted in Figure 3.

We have now supplied the details of the extension already sketched above (1.87). We proceed by verifying property (ii), cf. (1.87)-(1.91). Since we already know that $\phi_{r}^{0}:\left\langle M_{r}^{0}, \omega_{R}\right\rangle \rightarrow\left\langle\hat{M}_{r}^{0},-\omega_{R}\right\rangle$ is a $C^{\omega}$ symplectomorphism-a fact that can be verified from $\langle 4.56)-(4.58)$, in principle-we need only check that $\phi$ is $C^{\omega}$ at the 4 points in $M \backslash M_{r}^{0}$. This can be seen by inspection, provided one employs a suitable representation for $\phi$. Specifically, using (4.56)-(4.58) and (1.88) we find that for points in the patch $\mathscr{P}_{0}=\left\{\left(1, z_{1}\right) \mid z_{1} \in \mathbf{C}\right\}$ the image $\hat{m}=\phi(1, w)$ can be written

$$
\hat{m}=(1, \sigma(w)) \in \mathscr{P}_{0}, \quad w \neq-1
$$


where

$$
\begin{gathered}
\sigma \equiv \frac{[\cos d(w)+i \rho(w)(\operatorname{Im} w) \sin d(w)]}{\left[\rho(w)^{2}(\operatorname{Re} w)^{2}+1\right]^{1 / 2}}\left(\frac{\hat{d}(w)-\tau}{\left(\sin ^{2} \hat{d}(w)-\sin ^{2} \tau\right)(\pi-\tau-\hat{d}(w))}\right)^{1 / 2} \\
d \equiv \frac{\pi}{2}-\left(\frac{\pi}{2}-\tau\right)\left(1-|w|^{2}\right) /\left(1+|w|^{2}\right) \\
\rho \equiv\left(\frac{\sin ^{2} d(w)-\sin ^{2} \tau}{(\operatorname{Re} w)^{2} \sin ^{2} \tau+(\operatorname{Im} w)^{2} \sin ^{2} d(w)}\right)^{1 / 2} \\
\hat{d} \equiv \frac{1}{2 i} \operatorname{Ln}\left(\frac{\rho(w) \operatorname{Re} w+i}{\text { c.c. }}\right), \quad \operatorname{Ln}(-1) \equiv i \pi
\end{gathered}
$$

(with c.c. $\equiv$ complex conjugate). Alternatively, we have

$$
\hat{m}=(\kappa(w), 1) \in \mathscr{P}_{1}, \quad w \neq 1
$$

where

$\kappa \equiv \frac{[\cos d(w)-i \rho(w)(\operatorname{Im} w) \sin d(w)]}{\left[\rho(w)^{2}(\operatorname{Re} w)^{2}+1\right]^{1 / 2}}\left(\frac{\pi-\tau-\hat{d}(w)}{\left(\sin ^{2} \hat{d}(w)-\sin ^{2}(\pi-\tau)\right)(\hat{d}(w)-\tau)}\right)^{1 / 2}$

(Note that $\sigma(w) \kappa(w)=1, w \neq \pm 1$, as should be the case.) Now from (4.62) one easily sees that $\operatorname{Re} \sigma, \operatorname{Im} \sigma$ are $C^{\omega}$ functions of $\operatorname{Re} w, \operatorname{Im} w$ at $w=0,1$; similarly, from (4.67) it follows that $\operatorname{Re} \kappa, \operatorname{Im} \kappa$ are $C^{\omega}$ at $w=0,-1$. (Note these 3 points are the points $c, a, b$, resp., in Figure 3.) Substituting $w \rightarrow 1 / w$ in the above one readily verifies that $\phi$ is $C^{\omega}$ at $m=(0,1) \in \mathscr{P}_{1}$, too. (This is the point $d$ in Figure 3.)

Of course, we may just as well view $\phi$ as a $C^{\omega}$ antisymplectomorphism of the symplectic manifold $\left\langle\mathbb{P}^{1}, \omega_{R}\right\rangle$. Note that the self-duality property $(2.120)$ then entails $\phi \circ \phi=\mathrm{id}\left(\mathbf{P}^{1}\right)$, and that $\phi$ depends solely on $\tau \in(0, \pi / 2)$. Finally, we point out that $\phi$ has two fixed points at $m=(1, \pm i$ ) (the points $p$ and $q$ in Figure 3 ); these points lie on a Jordan curve $\Gamma \subset M_{r}^{0}$ of fixed points given by

$$
\Gamma=\left\{(\delta, \gamma) \in M^{0}|Q \cos | \beta|\gamma=\cot | \mu \mid \delta\right\}
$$

(This readily follows from (4.56)-(4.58).)

We proceed by studying the general case. First, consider the function $M^{0} \rightarrow$ $\mathbf{R}, w \longmapsto\left|w_{\nu}\right|^{2} /\left(1+\left|w_{1}\right|^{2}+\cdots+\left|w_{n}\right|^{2}\right)$, where $\nu \in\{0,1, \ldots, n\}$. This function 
may be viewed as the restriction of the function $\mathbf{P}^{n} \rightarrow \mathbf{R},\left(z_{0}, \ldots, z_{n}\right) \longmapsto\left|z_{\nu}\right|^{2} /(z$, $z$ ) to $M^{0} \subset \mathscr{P}_{0}$. The latter function is manifestly $C^{\omega}$ on $\boldsymbol{P}^{n}$. (To avoid any misunderstandings, let us recall what this means : Setting $z_{\sigma}=1$, it is real-analytic as a function of the real and imaginary parts of $z_{0}, \ldots, z_{\sigma-1}, z_{\sigma+1}, \ldots, z_{n} \in \mathbf{C}$, for any $\sigma \in\{0,1, \ldots, n\}$.$) From (1.97) we now see that the functions \delta_{\nu}, \nu=0, \ldots, n$, extend to $C^{\omega}$ functions on $M$, again denoted $\delta_{\nu}$. Thus, the matrix $A(q)$ (with $\left.\Sigma q_{j}=0\right)$ extends to a $C^{\omega}$ function on $M$, cf. (1.29). Similarly, the matrix elements $C(\mu, \beta, g ; q)_{j k}, j \neq k+1$, and

$$
\begin{aligned}
L_{k+1, k}= & \frac{\sin \tau}{\sin \frac{|\mu|}{2}\left(q_{k+1}-q_{k}\right)} \\
& \cdot \prod_{l \neq k, k+1}\left(\frac{\sin \left(\frac{|\mu|}{2}\left(q_{k+1}-q_{l}\right)+\tau\right)}{\sin \frac{|\mu|}{2}\left(q_{k+1}-q_{l}\right)} \frac{\sin \left(\frac{|\mu|}{2}\left(q_{k}-q_{l}\right)-\tau\right)}{\sin \frac{|\mu|}{2}\left(q_{k}-q_{l}\right)}\right)^{1 / 2}
\end{aligned}
$$

where $k=1, \ldots, N$ (cf. (2.82)), have $C^{\omega}$ extensions to $M$. (Here and below, we use $\bmod N$ notation. Thus, e.g., $q_{N+1}=q_{1}$.)

Consider now the vector-valued functions $f$ and $g$ given by (2.85) and (2.86), resp. These can be rewritten

$$
\begin{aligned}
& f_{1}=e^{\frac{\mu}{2} q_{1}}\left(\frac{\sin \left(|\mu| \delta_{0}-\tau\right)}{\sin |\mu| \delta_{0}}\right)^{1 / 2} \prod_{l \neq N, 1}\left(\frac{\sin \left(\frac{|\mu|}{2}\left(q_{1}-q_{l}\right)+\tau\right)}{\sin \frac{|\mu|}{2}\left(q_{1}-q_{l}\right)}\right)^{1 / 2} \\
& f_{j}=e^{\frac{\mu}{2} q_{j}} \bar{w}_{j-1}\left(\frac{\left(|\mu| \delta_{0}-\tau\right) \sin \left(|\mu| \delta_{j-1}-\tau\right)}{\left(|\mu| \delta_{j-1}-\tau\right) \sin |\mu| \delta_{j-1}}\right)^{1 / 2} \prod_{l \neq j-1, j}\left(\frac{\sin \left(\frac{|\mu|}{2}\left(q_{j}-q_{l}\right)+\tau\right)}{\sin \frac{|\mu|}{2}\left(q_{j}-q_{l}\right)}\right)^{1 / 2} \\
& j=2, \ldots, N \\
& g_{k}=e^{\frac{\mu}{2} q_{k}} w_{k}\left(\frac{\left(|\mu| \delta_{0}-\tau\right) \sin \left(|\mu| \delta_{k}-\tau\right)}{\left(|\mu| \delta_{k}-\tau\right) \sin |\mu| \delta_{k}}\right)^{1 / 2} \prod_{l \neq k, k+1}\left(\frac{\sin \left(\frac{|\mu|}{2}\left(q_{k}-q_{l}\right)-\tau\right)}{\sin \frac{|\mu|}{2}\left(q_{k}-q_{l}\right)}\right)^{1 / 2} \\
& k=1, \ldots, n \\
& g_{N}=e^{\frac{\mu}{2} q_{N}}\left(\frac{\sin \left(|\mu| \delta_{0}-\tau\right)}{\sin |\mu| \delta_{0}}\right)^{1 / 2} \prod_{l \neq N, 1}\left(\frac{\sin \left(\frac{|\mu|}{2}\left(q_{N}-q_{l}\right)-\tau\right)}{\sin \frac{|\mu|}{2}\left(q_{N}-q_{l}\right)}\right)^{1 / 2}
\end{aligned}
$$


Clearly, the exponentials and the factors in the products extend to $C^{\omega}$ functions on M. Moreover, substituting

$$
w_{\nu} \rightarrow \frac{z_{\nu}}{z_{0}}, \quad|\mu| \delta_{\nu}-\tau \rightarrow \frac{\left|z_{\nu}\right|^{2}(\pi-N \tau)}{(z, z)}, \quad \nu=0,1, \ldots, n
$$

in the remaining terms, one readily checks that the functions $f_{j} g_{k}, j, k=1, \ldots, N$, have $C^{\omega}$ extensions. Therefore, the Lax matrix $L$ admits a $C^{\omega}$ extension to $M$ (recall $L_{j k}$ equals $f_{j} C_{j k} g_{k}$ on $M^{0}$.)

On the other hand, although the functions $f$ and $g$ have $C^{\omega}$ extensions to $\mathscr{P}_{0} \subset$ $M$, they do not even admit a continuous extension to all of $M$. This is due to the factors $\left|z_{0}\right| \bar{z}_{0}$ in (4.71) and $\left|z_{0}\right| / z_{0}$ in (4.72); similarly, the factors $\sin \left(|\mu| \delta_{0}\right.$ $-\tau)^{1 / 2}$ in (4.70) and (4.73) are $C^{0}$ but not $C^{1}$ for $\left|z_{0}\right| \rightarrow 0$. To cope with this difficulty, we introduce the phases

$$
\rho_{\nu} \equiv \frac{1}{w_{\nu}}\left(\frac{|\mu| \delta_{\nu}-\tau}{|\mu| \delta_{0}-\tau}\right)^{1 / 2}=\frac{z_{0}\left|z_{\nu}\right|}{z_{\nu}\left|z_{0}\right|}, \quad \nu=0, \ldots, n
$$

and define

$$
f^{(\nu)} \equiv \overline{\rho_{\nu}} f, \quad g^{(\nu)} \equiv \rho_{\nu} g, \quad \nu=0, \ldots, n
$$

Then $f^{(\nu)}$ and $g^{(\nu)}$ have $C^{\omega}$ extensions to the patch $\mathscr{P}_{\nu} \subset M$. This is crucial in the proof of the following theorem, for which we are now prepared.

Theorem 4.6. The maps $\phi_{r}^{0}$ and $\mathrm{b}_{r}^{0}$ extend to mutually inverse $C^{\omega}$ symplectomorphisms $\phi$ and $\mathrm{b}$ between $\left\langle M, \omega_{R}\right\rangle$ and $\left\langle\hat{M},-\omega_{R}\right\rangle$. Employing homogeneous coordinates on $\hat{M}^{f} \simeq \mathbb{P}^{n}$ one has

$$
\mathrm{b}\left(e_{\nu}\right)=\varepsilon_{\nu}, \quad \nu=0, \ldots, n
$$

where

$$
\varepsilon_{\nu} \equiv\left(1, \omega^{-\nu}, \omega^{-2 \nu}, \ldots, \omega^{-n \nu}\right), \quad \omega \equiv e^{2 \pi i / N}
$$

and where $e_{0}, \ldots, e_{n}$ is the standard basis of $\mathbf{C}^{N}$. The varieties

$$
\hat{M}^{f e} \equiv \hat{M}^{f} \backslash \hat{M}^{f 0}
$$

and $\hat{M}_{b}^{f 0}$ have codimension 2. The manifolds $\hat{M}_{r}^{f 0}$ and $\hat{\Omega}_{r}^{f c}$ are connected, but not simply-connected. Finally, identifying $M$ and $\hat{M}$ with $\mathbb{P}^{n}$, the map $\phi$ is an involutory $C^{\omega}$ antisymplectomorphism of $\left\langle\mathbb{P}^{n}, \omega_{R}\right\rangle$.

Proof. We follow the proof of Theorem 4.1 as far as possible. We have already 
shown that $L$ has a $C^{\omega}$ extension to $M$, which we denote again by $L$. Since $L$ is unitary and has simple eigenvalues whose distance is bounded away from 0 on $M^{0}$, the same holds true on $M$. Therefore, the vector $\hat{\theta} \in A_{N}^{b}$ has a $C^{\omega}$ extension to $M$ satisfying $\hat{\theta} \in\left(A_{N}^{b}\right)^{c l}$.

Next, we rewrite the lhs of (2.101) as

$$
\begin{aligned}
\left(U^{*} A U\right)_{k, k+1}= & \frac{\sin \tau}{\sin \frac{|\beta|}{2}\left(\hat{\theta}_{k+1}-\hat{\theta}_{k}\right)} \\
& \cdot \prod_{l \neq k, k+1}\left(\frac{\sin \frac{|\beta|}{2}\left(\hat{\theta}_{k}-\hat{\theta}_{l}-d\right)}{\sin \frac{|\beta|}{2}\left(\hat{\theta}_{k}-\hat{\theta}_{l}\right)} \frac{\sin \frac{|\beta|}{2}\left(\hat{\theta}_{k+1}-\hat{\theta}_{l}+d\right)}{\sin \frac{|\beta|}{2}\left(\hat{\theta}_{k+1}-\hat{\theta}_{l}\right)}\right)^{1 / 2}
\end{aligned}
$$

where $k=1, \ldots, n$. Inspecting the rhs of this formula, one sees that it has a non-zero $C^{\omega}$ extension to $M$. (Once more, it converges to -1 as $\hat{\theta}_{k}-\hat{\theta}_{k+1} \downarrow d$. Note that (4.80) holds for $k=N$, too, and that the rhs then converges to 1 for $\hat{\theta}_{1}$ $-\hat{\theta}_{N} \uparrow 2 \pi /|\beta|-d$. $)$ Thus, when we introduce a function $\widetilde{U}: M \rightarrow U(N)$ obeying

$$
\begin{gathered}
\tilde{U}^{*} L \tilde{U}=\operatorname{diag}\left(e^{\beta \hat{\theta}_{1}}, \ldots, e^{\beta \hat{\theta}_{N}}\right), \quad \hat{\theta} \in\left(\boldsymbol{A}_{N}^{b}\right)^{c l}, \quad \sum_{j=1}^{N} \hat{\theta}_{j}=0 \\
\left(\tilde{U}^{*} A \widetilde{U}\right)_{k, k+1}<0
\end{gathered}
$$

then $\widetilde{U}$ is determined up to an overall phase ambiguity.

We proceed by fixing this phase in each patch $\mathscr{P}_{\nu}$, yielding $N$ unitaries $U_{0}, \ldots$, $U_{n}$. Specifically, we require that the first non-zero quantity in the sequence $\left(U_{\nu}^{t} g^{(\nu)}\right)_{1}, \ldots,\left(U_{\nu}^{t} g^{(\nu)}\right)_{N}$ be positive. (This makes sense : (4.76) yields a $C^{\omega}$ vector $g^{(\nu)}$ on $\mathscr{P}_{\nu} ;$ the paragraph containing $(2.100)$ entails

$$
\left\|f^{(\nu)}\right\|^{2}=\left\|g^{(\nu)}\right\|^{2}=\frac{\sin N \tau}{\sin \tau}
$$

on the dense subset $M_{r}^{0}$ of $\mathscr{P}_{\nu}$; hence (4.83) holds on $\mathscr{P}_{\nu}$, so $U_{\nu}^{t} g^{(\nu)} \neq 0$ on $\mathscr{P}_{\nu}$.) Then the unitaries thus defined on $\mathscr{P}_{\nu}$ are related to the unitary $U$ defined in Section 2.3 by

$$
U_{\nu}=\overline{\rho_{\nu}} U, \quad \nu=0, \ldots, n \quad\left(\text { on } M_{r}^{0}\right)
$$

(To see this, recall $\left(U^{t} g\right)_{1}>0$ on $M_{r .}^{0}$ )

Next, we fix $m \in \mathscr{P}_{\nu}$, a ball $B \subset \mathscr{P}_{\nu}$ around $m$ (w.r.t. the Fubini-Study metric) and a $C^{\omega}$ map $\mathscr{D}: B \rightarrow G L(N, \mathbf{C})$ satisfying

$$
\mathscr{D}^{-1} L \mathscr{D}=\operatorname{diag}\left(\lambda_{1}, \ldots, \lambda_{N}\right), \quad \lambda_{j}(m)=e^{\beta \hat{\theta}_{j}(m)}, \quad j=1, \ldots, N
$$


Then it follows as before that (4.14) holds true on $B$, provided $U$ is replaced by $U_{\nu}$. With this replacement the paragraph containing (4.18) applies, too, the result being that we obtain a renormalized $C^{\omega}$ function $\mathscr{D}_{r}$ on $B$ of the form

$$
\mathscr{D}_{r}=\chi U_{\nu}, \quad \chi: B \longrightarrow \mathbf{C}^{*}
$$

We continue by introducing

$$
\hat{g}_{r} \equiv \mathscr{D}_{r}^{t} g^{(\nu)}
$$

Clearly, $\hat{g}_{r}$ is $C^{\omega}$ in $B$ and $\hat{g}_{r} \neq 0$ on $B$. Consider now the function $\hat{z}: B \rightarrow \mathbb{C}^{N}$ defined by

$$
\hat{z}_{k-1} \equiv \overline{\hat{g}}_{r k} e^{\beta \hat{\theta}_{k} / 2}\left(\frac{\left(|\beta| \hat{\delta}_{k-1}-\tau\right) \sin |\beta| \hat{\delta}_{k-1}}{\sin \left(|\beta| \hat{\delta}_{k-1}-\tau\right)}\right)^{1 / 2} \prod_{l \neq k-1, k}\left(\frac{\sin \frac{|\beta|}{2}\left(\hat{\theta}_{k}-\hat{\theta}_{l}\right)}{\sin \frac{|\beta|}{2}\left(\hat{\theta}_{k}-\hat{\theta}_{l}+d\right)}\right)^{1 / 2}
$$

where $k=1, \ldots, N$. (Recall our standing $\bmod N$ convention, entailing $\hat{\theta}_{0}=\hat{\theta}_{N}$ ) The quotients in brackets are all $C^{\omega}$ and positive on $M$, so $\hat{z}$ is $C^{\omega}$ and $\neq 0$ in $B$. Furthermore, on the set $B_{r}^{0} \equiv B \cap M_{r}^{0}$ we have

$$
\hat{g}_{r}=\chi U_{\nu}^{t} g^{(\nu)}=\chi \overline{\rho_{\nu}} U^{t} g^{(\nu)}=\chi U^{t} g=\chi \hat{g}
$$

cf. (4.86), (4.84), (4.76). From $\hat{g}_{1} \neq 0$ on $B_{r}^{0}$ we now deduce $\hat{z}_{0} \neq 0$ on $B_{r}^{0}$. Therefore, the quantities

$$
\hat{w}_{i} \equiv \hat{z}_{i} / \hat{z}_{0}, \quad i=1, \ldots, n
$$

are well defined on $B_{r}^{0}$; moreover, they coincide with the $\hat{w}_{i}$ in (1.93), as follows from the formula (2.104) for $\hat{g}$.

The point is now, that $\hat{z}$ may be regarded as supplying homogeneous coordinates for a function $\phi: B \rightarrow \hat{M} \simeq \mathbb{P}^{n}$ (since $\hat{z} \neq 0$ on $\left.B\right)$. This function is $\mathbb{C}^{\omega}$ in $B$ (since $\hat{z}$ is), and it coincides with the (reparametrized) map $\phi_{r}^{0}$ on the dense set $B_{r}^{0}$ (as we have seen in the previous paragraph). But then it is routine to deduce that $\phi_{r}^{0}$ extends to a $C^{\omega}$ map $\phi$ from $M$ into $\hat{M}$. By virtue of self-duality, the analogous conclusion for $b_{r}^{0}$ is plain. Therefore, we may conclude that $\phi$ maps onto $\hat{M}$ and has a $C^{\omega}$ inverse b extending $b_{r}^{0}$. Using Theorem 3.5, connectedness of $\hat{M}^{f}$, and real-analyticity of $\phi$ and $b$, the first assertion of the theorem now easily follows. Then the last assertion is clear from self-duality. 
Next, we prove (4.77). The points $\varepsilon_{\nu}$ belong to $M^{0}$ and have coordinates $\delta_{j}\left(\varepsilon_{\nu}\right)=\pi / N|\mu|, \quad \gamma_{j}\left(\varepsilon_{\nu}\right)=-2 \pi \nu j / N|\beta|(\bmod 2 \pi /|\beta|), \quad j=1, \ldots, n$ in view of (1.93). By (2.82) and (A.29) this entails $L\left(\varepsilon_{\nu}\right)=\operatorname{diag}\left(1, \omega^{\nu}, \ldots, \omega^{\nu n}\right) E \operatorname{diag}\left(\omega^{-\nu}, \omega^{-2 \nu}, \ldots, 1\right) \sim \omega^{-\nu} E, \quad \nu=0, \ldots, n$

where $\sim$ denotes similarity. Using (A.32) and $\sum \hat{\theta}_{j}=0$, it is then straightforward to verify

$$
\hat{\delta}_{\sigma}\left(\varepsilon_{\nu}\right)=d / 2, \quad \sigma \neq \nu, \quad \hat{\delta}_{\nu}\left(\varepsilon_{\nu}\right)=\pi /|\beta|-n d / 2
$$

(recall (1.94)). Now we choose a sequence $p_{k} \in M_{r}^{0}$ converging to $\varepsilon_{\nu}$ as $k \rightarrow \infty$. Using (1.93) to write

$$
\phi\left(p_{k}\right)=\left(\left(\hat{\delta}_{0}\left(p_{k}\right)-d / 2\right)^{1 / 2}, \ldots, e^{\mu \hat{\gamma}_{t}\left(p_{k}\right)}\left(\hat{\delta}_{i}\left(p_{k}\right)-d / 2\right)^{1 / 2}, \ldots\right)
$$

it follows from (4.93) and the continuity of $\phi$ (already established above) that $\phi$ $\left(\varepsilon_{\nu}\right)=e_{\nu}$, which entails (4.77).

We continue by proving

$$
\operatorname{codim} \hat{M}_{b}^{0}=2
$$

To this end we first observe that the variety $M^{e}$ defined by (4.79) amounts to $\mathbf{P}^{n} \backslash$ $\mathrm{C}^{* n}$, and so has codimension 2 . Second, we note that

$$
\hat{M}_{b}^{0} \subset \phi\left(M^{e}\right)
$$

so that $\operatorname{codim} \hat{M}_{b}^{0} \geq 2$. Third, we assert that there exists an open neighborhood $N_{\nu}$ $\subset M$ of $e_{\nu}$ such that

$$
\phi\left(N_{\nu}\right) \subset \hat{M}^{0}
$$

Indeed, (4.77) and self-duality entail $\phi\left(e_{\nu}\right)=\varepsilon_{\nu}$, so existence follows from $\varepsilon_{\nu} \in \hat{M}^{0}$ and $\phi$ being a homeomorphism. Fourth, we define

$$
N_{\nu}^{e} \equiv N_{\nu} \cap M^{e}, \quad N_{\nu}^{0} \equiv N_{\nu} \cap M^{0}
$$

From $e_{\nu} \in N_{\nu}^{e}$ we then deduce $\operatorname{codim} N_{\nu}^{e}=2$. But due to (4.97) we have $\phi\left(N_{\nu}^{e}\right) \subset$ $\hat{M}_{b}^{0}$, implying codim $\hat{M}_{b}^{0} \leq 2$. Hence, (4.95) results.

Since $\hat{M}^{0}$ is connected, it now follows that $\hat{M}^{0} \backslash \hat{M}_{b}^{0}=\hat{M}_{r}^{0}$ is connected. 
Therefore, it remains to show that $\hat{M}_{r}^{0}$ is not simply-connected. To this end we observe that the commutative diagram (1.85) may be invoked. (Indeed, the components occurring in Theorem 3.5 are equal to the spaces in the diagram due to the connectedness of $\hat{M}_{r}^{0}$ just established.) Since $\pi\left(\hat{E}_{N}\right)$ is a regular covering projection, we need only prove that $\tilde{\Omega}_{r}$ is not simply-connected. Because $R$ is $C^{\omega}$ in $\tilde{\Omega}_{r}$, it suffices to show that the $\theta$-part of $R$ is multi-valued.

To prove this, consider the set $N_{\nu}^{0}$ defined by (4.98). Due to (4.97) this set is a subset of $M_{r}^{0}$. Therefore, $M_{r}^{0}$ contains the tori $\left\{(\delta, \gamma) \mid \gamma \in(-\pi /|\beta|, \pi /|\beta|]^{n}\right\}$ for $\delta \in \mathrm{a}_{n}^{b}$ sufficiently close to the alcove corner $\delta\left(e_{\nu}\right)$. But as $\exp \left(\beta \gamma_{j}\right)$ winds counterclockwise once around $S^{1}$, the corresponding $\theta_{j}$ and $-\theta_{j+1}$ clearly increase by $2 \pi /|\beta|$, cf. (1.85), (1.30).

Corollary 4.7. The maps $\tilde{\Phi}$ and $\tilde{\mathscr{E}}$ extend to mutually inverse $C^{\omega}$ symplectomorphisms $\tilde{\Phi}^{\times} \equiv P_{2} \times \phi$ and $\tilde{\mathscr{E}}^{\times} \equiv P_{2} \times \mathrm{b}$ between $\left\langle\Omega^{\# c}, \omega^{\# c}\right\rangle$ and $\left\langle\hat{\Omega}^{\# c}, \hat{\omega}^{\# c}\right\rangle$, where

$$
\begin{aligned}
& \Omega^{\# c} \equiv \mathbb{R}^{2} \times M, \quad \omega^{\# c} \equiv N d \delta_{s} \wedge d \gamma_{s}+\omega_{R} \\
& \hat{\Omega}^{\# c} \equiv \mathbf{R}^{2} \times \hat{M}, \quad \hat{\omega}^{\# c} \equiv N d \hat{\gamma}_{s} \wedge d \hat{\delta}_{s}-\omega_{R}
\end{aligned}
$$

Proof. Obvious from Theorem 4.6.

Next, we supply the details of the diagram (1.86) and its extension (1.102). We begin by recalling that the first $\mathbf{Z}$-factor in the $\mathbf{Z} \times \mathbf{Z}$-action on $\mathbf{R}^{2} \times M^{0}$ has generator $G$ given by (2.39). The second factor corresponds to the quotient group $\mathbb{Z}^{N} / E_{N}$, cf. (1.42). One easily checks that its generator reads

$G_{q}:\left(\delta_{s}, \gamma_{s} ; \delta, \gamma\right) \longmapsto\left(\delta_{s}, \gamma_{s}+2 \pi / N|\beta| ; \delta, \gamma_{1}-2 \pi / N|\beta|, \ldots, \gamma_{n}-2 \pi n / N|\beta|\right)$

Recalling self-duality, it should cause no surprise that $G_{q}$ equals the map (2.40) when hats are omitted and $\mu$ is replaced by $\beta$. By the same token, the dual generator $\hat{G}_{q} \equiv \tilde{\Phi} \circ G_{q} \circ \tilde{\mathscr{E}}$ is given by (2.39) with hats added and $\mu \rightarrow \beta$. Since these generators map $\hat{\Omega}_{r}^{f c}$ onto itself, the projections in (1.86) are well defined. The map $\Phi$ is now defined so as to ensure commutativity.

We proceed by introducing

$$
\begin{gathered}
\mathrm{g}: \mathbb{P}^{n} \longrightarrow \mathbb{P}^{n},\left(z_{0}, z_{1}, \ldots, z_{n}\right) \longmapsto\left(z_{n}, z_{0}, z_{1}, \ldots, z_{n-1}\right) \\
\mathbf{g}_{q}: \mathbf{P}^{n} \longrightarrow \mathbb{P}^{n},\left(z_{0}, z_{1}, \ldots, z_{n}\right) \longmapsto\left(z_{0}, \omega^{-1} z_{1}, \ldots, \omega^{-n} z_{n}\right), \omega \equiv e^{2 \pi / N}
\end{gathered}
$$

One readily checks that these maps are symplectomorphisms w.r.t. $\omega_{R}$ whose $N$ th 
powers equal the identity. Moreover, viewing $\boldsymbol{M}^{0}$ as $\mathbf{C}^{{ }^{* n}} \subset \mathbf{P}^{n}$ via (1.93), we have

$$
\begin{gathered}
G\left(\delta_{s}, \gamma_{s} ; m\right)=\left(\delta_{s}+2 \pi / N|\mu|, \gamma_{s} ; \mathbf{g}(m)\right) \\
G_{q}\left(\delta_{s}, \gamma_{s} ; m\right)=\left(\delta_{s}, \gamma_{s}+2 \pi / N|\beta| ; \mathbf{g}_{q}(m)\right)
\end{gathered}
$$

Therefore, $\boldsymbol{G}$ and $G_{q}$ extend to symplectomorphisms of $\left\langle\Omega^{\# c}, \omega^{\# c}\right\rangle$, with duals that are obvious by now. The corresponding group actions are clearly free, so we obtain symplectic manifolds

$$
\hat{\Omega}^{f \#} \equiv \hat{\Omega}^{f \# c} / \hat{\mathbf{Z}}^{f} \times \hat{\mathbf{Z}}^{f}, \quad \hat{\omega}^{f \#} \equiv \hat{\omega}^{f \# c} / \hat{\mathbf{Z}}^{f} \times \hat{\mathbf{Z}}^{f}
$$

Note that $\Omega^{\#}$ may be viewed as a fiber bundle over

$$
\mathbf{T}^{2} \simeq\left\{\left(\delta_{s}, \gamma_{s}\right) \in[0,2 \pi / N|\mu|) \times[0,2 \pi / N|\beta|)\right\}
$$

with fiber $\mathbf{P}^{n}$ and transition functions $\mathbf{g}$ and $\mathbf{g}_{\mathrm{q}}$ w.r.t. the $\delta_{s}$ and $\gamma_{s}$ tori, resp. The map $\Phi^{\times}$in the diagram (1.102) is now defined such that the diagram commutes.

Corollary 4.8. The maps $\Phi$ and $\Phi^{\times}$in (1.86) and (1.102) are symplectomorphisms from $\left\langle\Omega_{r}, \omega\right\rangle$ onto $\left\langle\hat{\Omega}_{r}, \hat{\omega}\right\rangle$ and from $\left\langle\Omega^{\#}, \omega^{\#}\right\rangle$ onto $\left\langle\hat{\Omega}^{\#}, \hat{\omega}^{\#}\right\rangle$, resp.

Proof. Clear from the above.

\subsection{The $\mathrm{III}_{\mathrm{b}} \operatorname{Map} \phi$}

In this section we shall obtain more information on the reduced harmonic oscillator map $\phi$, viewed as an involutory antisymplectomorphism of $\left\langle\mathbf{P}^{n}, \omega_{\text {ren }}\right\rangle$, cf. (1.103). To this end we introduce the involutory antisymplectomorphisms

$$
\begin{gathered}
\mathrm{k}:\left(z_{0}, \ldots, z_{n}\right) \longmapsto\left(\bar{z}_{0}, \ldots, \bar{z}_{n}\right) \\
\hat{\mathrm{k}}:\left(z_{0}, \ldots, z_{n}\right) \longmapsto\left(\bar{z}_{0}, \bar{z}_{n}, \ldots, \bar{z}_{1}\right)
\end{gathered}
$$

and the involutory symplectomorphism

$$
\mathrm{p} \equiv \mathrm{k} \circ \hat{\mathrm{k}}=\hat{\mathrm{k}} \circ \mathrm{k}:\left(z_{0}, z_{1}, \ldots, z_{n}\right) \longmapsto\left(z_{0}, z_{n}, \ldots, z_{1}\right)
$$

Then we have

$$
\phi \circ \mathrm{k}=\hat{\mathrm{k}} \circ \phi, \quad \phi \circ \hat{\mathrm{k}}=\mathrm{k} \circ \phi, \quad \phi \circ \mathrm{p}=\mathrm{p} \circ \phi
$$


by virtue of (2.119). Next, we set

$$
\mathbf{t} \equiv \mathbf{g} \circ \mathbf{g}_{q}=\mathbf{g}_{q} \circ \mathbf{g}:\left(z_{0}, \ldots, z_{n}\right) \longmapsto\left(z_{n}, \omega^{-1} z_{0}, \ldots, \omega^{-n} z_{n-1}\right)
$$

where we used (4.102), (4.103). One readily verifies

$$
p \circ t \circ p=t^{-1}
$$

so $\mathrm{p}$ and $\mathrm{t}$ generate a dihedral group action on $\mathbf{P}^{n}$. Using (4.111) and the relations

$$
\phi \circ \mathrm{g}=\mathrm{g}_{q} \circ \phi, \quad \phi \circ \mathrm{g}_{q}=\mathrm{g} \circ \phi, \quad \phi \circ \mathrm{t}=\mathrm{t} \circ \phi
$$

(which follow from (2.40)), we deduce that $\phi$ commutes with this action.

Clearly, the generators $\mathrm{g}, \mathrm{g}_{q}, \mathrm{t}$ and $\mathrm{p}$ are isometries of the Riemannian manifold $\left\langle\mathbb{P}^{n}, g_{F S}\right\rangle$, where $g_{F S}$ denotes the Fubini-Study metric. Indeed, they may be viewed as pushdowns of unitaries on $\mathbf{C}^{N}$ whose action on $\mathbf{C}^{N}$ can be read off from (4.102), (4.103), (4.112) and (4.110), resp. For the first three cases the eigenvalues of these unitaries are the $N$ th roots of 1 , and the corresponding eigenspaces give rise to $N$ fixed points. Obviously, for $g$ and $\mathrm{g}_{q}$ these are given by $\varepsilon_{\nu}$ and $e_{\nu}$, $\nu=0, \ldots, n$, resp. Introducing

$$
p_{\nu k} \equiv \exp (i \pi k(N+2 \nu-k) / N), \quad k=0, \ldots, n, \quad \nu \in \mathbb{Z}
$$

(so that $p_{\nu+N}=p_{\nu}$ ), one readily checks that $\mathrm{t}$ has fixed points $p_{0}, \ldots, p_{n}$.

Next, we introduce the fixed-point space

$$
\Gamma \equiv\left\{z \in \mathbb{P}^{n} \mid \phi(z)=z\right\}
$$

Since we have

$$
\mathrm{p}\left(p_{\nu}\right)=p_{N-\nu}, \quad \nu=0, \ldots, n
$$

the only point $p_{\nu}$ that is fixed under $\mathrm{p}$ for any $N$ is $p_{0}$, whereas for $N$ even we obtain an extra fixed point $p_{N / 2}$. Now $\phi$ commutes with the dihedral group action generated by $\mathrm{t}$ and $\mathrm{p}$, and for $N$ odd we have just established that $p_{0}$ is the only fixed point under this action. Thus we must have $p_{0} \in \Gamma$ for $N$ odd, whereas for $N$ even we can only conclude that $\phi$ leaves the set $\left\{p_{0}, p_{N / 2}\right\}$ invariant.

We now observe that the points $p_{\nu}$ have already appeared above, cf. the paragraphs containing (3.60) and (3.63). In particular, using (2.117) and (1.93) one sees that $p_{0}$ amounts to the point $P_{0}(0,0)$. Thus, the above yields a new and completely algebraic proof of (2.140) for $N$ odd, as announced below (2.140). (Indeed, the developments after Corollary 4.8 can be rephrased in the context of 
Lemma 2.3.) In view of (3.49) we actually have

$$
p_{0} \in \Gamma
$$

for any $N$, which entails

$$
p_{N / 2} \in \Gamma \quad(N \text { even })
$$

Next, we observe that

$$
\mathrm{g}\left(p_{\nu}\right)=p_{\nu+1}, \quad \mathrm{~g}_{q}\left(p_{\nu}\right)=p_{\nu-1}
$$

and so

$$
\phi\left(p_{\nu}\right)=\left(\phi \circ \mathbf{g}^{\nu}\right)\left(p_{0}\right)=\left(\mathbf{g}_{q}^{\nu} \circ \phi\right)\left(p_{0}\right)=\mathbf{g}_{q}^{\nu}\left(p_{0}\right)=p_{N-\nu}
$$

Moreover, introducing the points

$$
\bar{p}_{\nu} \equiv \mathrm{k}\left(p_{\nu}\right)
$$

we have

$$
\phi\left(\bar{p}_{\nu}\right)=(\phi \circ \mathrm{k})\left(p_{\nu}\right)=(\hat{\mathrm{k}} \circ \phi)\left(p_{\nu}\right)=\hat{\mathrm{k}}\left(p_{N-\nu}\right)=(\mathrm{k} \circ \mathrm{p})\left(p_{N-\nu}\right)=\mathrm{k}\left(p_{\nu}\right)=\bar{p}_{\nu}
$$

where (4.110), (4.111) and (4.117) have been used. Thus,

$$
\bar{p}_{\nu} \in \Gamma, \quad \nu=0, \ldots, n
$$

Since the map $\phi$ is an antisymplectomorphism, its Lefschetz number equals $0 /$ 1 for $n$ odd/even. Thus, $\Gamma$ could have been empty for $n$ odd, a priori. Note that the fixed points are not Lefschetz, since $\phi$ is involutive.

We continue by introducing the symplectomorphism

$$
f \equiv k \circ \phi
$$

which satisfies

$$
f^{2}=p, \quad f^{4}=i d
$$

on account of (4.110) and (4.111). Using (4.56)-(4.58) one easily checks that for 
$n=1$ this map has 2 and only 2 fixed points at

$z_{ \pm}=\left(1, \pm([d(\tau)-\tau] /[\pi-\tau-d(\tau)])^{ \pm 1 / 2}\right), \quad d(\tau) \equiv \operatorname{Arcsin}\left((\sin \tau)^{1 / 2}\right)$

More generally, for any $n \geq 1$ it must have at least $N$ fixed points by virtue of Lefschetz theory. In view of the convergence result we are about to prove, it is plausible that the fixed-point space of $f$ has the same characteristics as the fixed-point space of the $\mathbb{P}^{n}$-automorphism $\boldsymbol{f}_{0}$ that is the quotient of

$$
F_{0} \equiv N^{-1 / 2}\left(\begin{array}{ccccc}
1 & 1 & 1 & \cdots & 1 \\
1 & \omega & \omega^{2} & \cdots & \omega^{n} \\
\vdots & \vdots & \vdots & \ddots & \vdots \\
1 & \omega^{n} & \omega^{2 n} & \cdots & \omega^{n^{2}}
\end{array}\right), \omega \equiv e^{2 \pi i / N}
$$

In particular, we expect 3 and only 3 fixed points for $N=3$, and 2 and only 2 isolated fixed points for $N=4$. (Note $F_{0}$ has eigenvalues $1,-1, i$ for $N=3,4$, the first one being degenerate for $N=4$.) After the following theorem we shall show that one of the $N=3$ fixed points is in fact $\tau$-independent.

Theorem 4.9. The symplectomorphism f obeys

$$
\lim _{\tau \uparrow \pi / N} f(z)=f_{0}(z), \quad \forall z \in \mathbf{P}^{n}
$$

where the limit refers to the Fubini-Study metric and is uniform on $\mathbb{P}^{n}$. There exists no $\tau \in(0, \pi / N)$ such that $f$ is the quotient of an invertible linear map on $\mathbf{C}^{N}$.

Proof. In view of (4.77) and (4.125) we have

$$
f\left(e_{\nu}\right)=\bar{\varepsilon}_{\nu}, \quad \nu=0, \ldots, n, \quad \forall \tau \in(0, \pi / N)
$$

(Recall b equals $\phi$ in the picture adopted after Corollary 4.8.) Now assume that $\tau_{0}$ $\in(0, \pi / N)$ and $M \in G L(N, \mathbf{C})$ exist such that $f$ is the pushdown of $M$. Then $M$ must satisfy

$$
M e_{\nu}=m_{\nu} \bar{\varepsilon}_{\nu}, \quad \nu=0, \ldots, n
$$

for certain $m_{\nu} \in \mathbf{C}^{*}$. Moreover, as $\mathrm{f}$ is symplectic, $M$ is unitary up to a scalar, so $\left|m_{0}\right|=\cdots=\left|m_{n}\right| \equiv \rho$. Recalling now $\mathrm{f}^{2}=\mathrm{p}$, it follows that $M^{2} e_{0}=N \rho^{2} e^{i \phi} e_{0}$, cf. (4.110). This is readily seen to entail $m_{\nu}=\rho e^{i \phi / 2}$, so we may as well take $M=F_{0}$, cf. (4.128).

Summarizing, the above assumption implies that $f$ equals $f_{0}$ for $\tau=\tau_{0}$. Now for $N=2$ the fixed points of $f_{0}$ are given by 


$$
z_{ \pm, 0} \equiv\left(1, \pm\left(2^{1 / 2}-1\right)^{ \pm 1}\right)
$$

Comparing with (4.127) we conclude

$$
a\left(\tau_{0}\right)=\left(3-2^{3 / 2}\right) \pi, \quad a(\tau) \equiv\left(2^{3 / 2}-2\right)\left(2^{1 / 2} d(\tau)-\tau\right)
$$

Since $a(\tau)$ increases from 0 to $\left(3-2^{3 / 2}\right) \pi$ as $\tau$ goes from 0 to $\pi / 2$, this contradicts $\tau_{0} \in(0, \pi / 2)$.

Next, let $N>2$ and consider $\operatorname{Tr} L$ evaluated in the point

$$
z_{x} \equiv(1, x, 0, \ldots, 0), \quad x \in \mathbf{R}
$$

Using (1.97) and (4.70)-(4.73) one obtains

$$
\begin{aligned}
\operatorname{Tr} L= & x(\sin r(x))^{1 / 2}\left(\frac{\sin \left(x^{2} r(x)\right)}{x^{2}} \cdot \frac{\sin (2 \tau+r(x)) \sin \left(2 \tau+x^{2} r(x)\right)}{\sin (\tau+r(x))^{2} \sin \left(\tau+x^{2} r(x)\right)^{2}}\right)^{1 / 2} \\
& \cdot \prod_{l \neq 1,2, N}\left(1-\frac{\sin ^{2} \tau}{\sin ^{2}\left(x^{2} r(x)+(l-1) \tau\right)}\right)^{1 / 2}
\end{aligned}
$$

where

$$
r(x) \equiv(\pi-N \tau) /\left(1+x^{2}\right)
$$

Clearly, $\operatorname{Tr} L$ can be analytically continued off the real axis. The function thus obtained is two-valued : it has a square-root branch point at $x=i(N \tau / \pi)^{1 / 2}$, e.g.

On the other hand, taking $x \neq-1$ one obtains from (4.128)

$$
f_{0}\left(z_{x}\right)=\left(1, \frac{1+x \omega}{1+x}, \ldots, \frac{1+x \omega^{n}}{1+x}\right)
$$

Using (the duals of ) (1.97) and (1.29) we can evaluate $\operatorname{Tr} \hat{L}$ in this point, yielding

$$
\operatorname{Tr} \hat{L}=\sum_{j=1}^{N} \exp \left(r_{j}(x, \tau)\right)
$$

where the functions $r_{j}$ are rational in $x$. Thus it follows that for $\tau=\tau_{0}$ the onevalued function (4.138) equals the two-valued function (4.135), a contradiction.

It remains to prove the first assertion. We begin by noting

$$
\lim _{\tau \uparrow \pi / N}|\mu| \delta_{\nu}=\pi / N, \quad \nu=0, \ldots, n
$$


uniformly on $\mathbf{P}^{n}$, cf. (1.97). Hence,

$$
\begin{gathered}
\lim _{\tau \uparrow \pi / N} A=\operatorname{diag}\left(e^{i \pi(N-1) / N}, e^{i \pi(N-3) / N}, \ldots, e^{i \pi(-N+1) / N}\right) \equiv A_{0} \\
\lim _{\tau \uparrow \pi / N} L=-S^{t} \equiv L_{0}
\end{gathered}
$$

uniformly on $\mathbb{P}^{n}$, cf. (4.30), (4.69)-(4.73), (A.35).

Next, we introduce the matrix

$$
\widetilde{U}_{0} \equiv \operatorname{Col}\left(u^{(1)}, \ldots, u^{(N)}\right)
$$

cf. (A.37). From $L_{0}=S^{N-1}$ and (A.36) one deduces

$$
\widetilde{U}_{0}^{*} L_{0} \widetilde{U}_{0}=A_{0}
$$

and using (A.37) one readily verifies

$$
\left(\widetilde{U}_{0}^{*} A_{0} \widetilde{U}_{0}\right)_{k, k+1}=-1, \quad k=1, \ldots, n
$$

Consider now the unitary $\widetilde{U}$ defined by (4.81), (4.82) up to an overall phase. We are going to prove that this phase can be chosen such that we have

$$
\lim _{\tau \uparrow \pi / N} \tilde{U}=\tilde{U}_{0}
$$

uniformly on $\mathbb{P}^{n}$. First, we observe that (4.141)-(4.143) entail that $L$ has eigenprojections $P_{j}$ converging to the projections on $u^{(j)}, j=1, \ldots, N$, as $\tau \uparrow \pi / N$. Furthermore, the convergence is uniform, since the limit (4.141) is uniform. Therefore, the vectors $a^{(j)} \equiv P_{j} u^{(j)}$ converge uniformly to the (constant) vectors $u^{(j)}, j=1, \ldots, N$. In particular, there exists $\varepsilon>0$ such that $a^{(j)}$ is non-zero for all $j \in\{1, \ldots, N\}, z \in \mathbb{P}^{n}$, and $\tau \in(\pi / N-\varepsilon, \pi / N]$. Restricting attention to this $\tau$-interval, we deduce that the vectors $b^{(j)} \equiv a^{(j)} /\left\|a^{(j)}\right\|$ yield an orthonormal base of eigenvectors of $L$ converging uniformly to $u^{(j)}, j=1, \ldots, N$. Therefore, the unitary matrix $U^{\prime} \equiv \operatorname{Col}\left(b^{(1)}, \ldots, b^{(N)}\right)$ converges uniformly to $\widetilde{U}_{0}$ as $\tau \uparrow \pi / N$.

Denoting now the columns of $\tilde{U}$ by $c^{(1)}, \ldots, c^{(N)}$, we may and will fix the phase ambiguity in $\widetilde{U}$ by requiring $c^{(1)}=b^{(1)}$; then one has $c^{(j)}=\chi_{j} b^{(j)}, j>1$, for certain phase functions $\chi_{j}: \mathbb{P}^{n} \rightarrow S^{1}$. We claim that this phase choice ensures (4.145), uniformly on $\mathbf{P}^{n}$.

To prove this claim, we need only show $\chi_{j} \rightarrow 1$ uniformly, since we already know $b^{(j)} \rightarrow u^{(j)}$ uniformly. To this end, we use (4.140) and (4.144) to infer $\left(U^{\prime *} A U^{\prime}\right)_{k, k+1} \equiv c_{k} \rightarrow-1$, uniformly on $\mathbb{P}^{n}$ (with $k=1, \ldots, n$ ). Next, we observe that $c_{k}=\chi_{k} \bar{\chi}_{k+1} n_{k}$, where $n_{k} \equiv\left(\tilde{U}^{*} A \tilde{U}\right)_{k, k+1}$ and $\chi_{1} \equiv 1$. Since $\left|c_{k}\right| \rightarrow 1$, we get 
$\left|n_{k}\right| \rightarrow 1$, and since $n_{k}<0$ (recall (4.82)), this entails $n_{k} \rightarrow-1, k=1, \ldots, n$. Hence we obtain successively $\chi_{2} \rightarrow 1, \ldots, \chi_{n} \rightarrow 1$, uniformly on $\mathbf{P}^{n}$, so the proof of (4.145) is now complete.

We continue by fixing $z \in \mathscr{P}_{\nu}$ and introducing the renormalized vectors

$$
g_{\text {ren }}^{(\nu)}(z) \equiv\left(\frac{\sin \tau}{\sin N \tau}\right)^{1 / 2} g^{(\nu)}(z), \quad \nu=0, \ldots, n
$$

cf. (4.75), (4.76), (4.83). Then we obtain

$$
\begin{aligned}
\lim _{\tau \uparrow \pi / N} g_{\text {ren }}^{(\nu)}(z) & =(z, z)^{-1 / 2} \frac{\left|z_{\nu}\right|}{z_{\nu}}\left(e^{i \pi(N-1) / 2 N} z_{1}, \ldots, e^{i \pi(-N+3) / 2 N} z_{n}, e^{i \pi(-N+1) / 2 N} z_{0}\right) \\
& \equiv \frac{\left|z_{\nu}\right|}{z_{\nu}} g_{r e n}(z), \quad z \in \mathscr{P}_{\nu}
\end{aligned}
$$

(Indeed, this follows from (4.139); note, in particular, that the product in (4.72) and (4.73) has limit 1.) Moreover, a straightforward calculation yields

$$
\begin{aligned}
\left(\tilde{U}_{0}^{t} g_{r e n}(z)\right)_{k} & =e^{i \pi(N+1-2 k) / 2 N} \sum_{\nu=0}^{n} \omega^{(k-1) \nu} z_{\nu} /[N(z, z)]^{1 / 2} \\
& =\frac{e^{i \pi(N+1-2 k) / 2 N}}{(z, z)^{1 / 2}}\left(F_{0}\left(z_{0}, z_{1}, \ldots, z_{n}\right)^{t}\right)_{k}, k=1, \ldots, N, \forall z \in \mathbf{P}^{n}
\end{aligned}
$$

We are now ready to exploit the paragraph containing (4.88). It entails that $\mathrm{f}=\mathrm{k} \circ \phi: B \rightarrow \mathbf{P}^{n}$ may be written

$$
f(z)_{k}=\left(\widetilde{U}^{t}(z) g_{r e n}^{(\nu)}(z)\right)_{k} \exp \left(-\beta \hat{\theta}_{k}(z) / 2\right) p_{k}(z), \quad k=1, \ldots, N
$$

where the function $p_{k}$ denotes the product of all square-root factors at the rhs of (4.88). Thus we have

$$
\lim _{\tau \uparrow \pi / N} p_{k}(z)=(\sin (\pi / N))^{1 / 2} \equiv p
$$

and using also (4.145), (4.147) and (4.148) we now obtain

$$
\lim _{\tau \uparrow \pi / N} f(z)=\frac{p}{(z, z)^{1 / 2}} \frac{\left|z_{\nu}\right|}{z_{\nu}} F_{0}\left(z_{0}, \ldots, z_{n}\right)^{t}, \quad \forall z \in B \subset \mathscr{P}_{\nu}
$$

w.r.t. the Fubini-Study metric. From this one easily deduces (4.129).

Finally, we prove the uniformity assertion. Since $\mathbf{P}^{n}$ is compact, it suffices to show that (4.151) holds uniformly for $z$ in an arbitrary compact $K \subset B$. Now the 
limit (4.147) is clearly uniform for $z \in K$, and so is the limit (4.150). Since the limits $\tilde{U}^{t}(z) \rightarrow \widetilde{U}_{0}^{t}$ and $\beta \tilde{\theta}_{k}(z) \rightarrow i \pi(N+1-2 k) / N$ are uniform on $\mathbf{P}^{n}$, it follows from (4.149) that (4.151) holds true uniformly on $K$.

For $n=1$ one obtains from (4.61)-(4.65)

$$
\begin{gathered}
\lim _{\tau \downarrow 0} \sigma(w)=\exp \left( \pm i \pi|w|^{2} /\left(1+|w|^{2}\right)\right)\left(\frac{|\operatorname{Arg} w|}{\pi-|\operatorname{Arg} w|}\right)^{1 / 2}, \pm \operatorname{Arg} w \in(0, \pi) \\
\lim _{\tau \downarrow 0} \sigma(w)=\left\{\begin{array}{cc}
0 & \pm w>0 \\
\infty &
\end{array}\right.
\end{gathered}
$$

Thus, though $\phi$ (and hence $f$ ) has a pointwise limit for $\tau \downarrow 0$, the limiting map is discontinuous. Probably, $f$ has a pointwise limit for $n>1$, too, but again the limiting map cannot be a diffeomorphism. One way to see this is to observe that

$$
\lim _{\tau \downarrow 0} f\left(1, w_{1}, \ldots, w_{n}\right)=e_{0}, \quad \forall w \in(0, \infty)^{n}
$$

(Indeed, from (1.93) and (2.82) one deduces that for these points one has $L \rightarrow \mathbb{1}_{N}$ as $\tau \downarrow 0$.)

For the last topic of this section it is convenient to write $V \equiv \mathbb{P}^{n}$ and use suffixes $0, r, b, e$ for $V$ just as we did for $M$ and $\hat{M}$. In particular, we have

$$
V^{0}=\mathbf{C}^{* n}, \quad V^{e}=V \backslash V^{0}, \quad V_{b}^{0}=V^{0} \cap \phi\left(V^{e}\right)
$$

so $V^{e}$ is the union of the $N$ complex hyperplanes $\mathrm{P}^{n-1} \subset V$ obtained by requiring $z_{0} z_{1} \cdots z_{n}=0$. Next, we introduce

$$
V_{b} \equiv \phi\left(V^{e}\right), \quad V_{b}^{e} \equiv V^{e} \cap V_{b}
$$

and note that $\phi$ may be replaced by $\mathrm{f}$ in these formulas. (Indeed, $\mathrm{k}$ and $\hat{\mathrm{k}}$ leave $V^{e}$ invariant.) As we have already seen, $V_{b}^{e}$ is empty for $n=1$. Now let $n>1$ and consider the projective variety

$$
V_{b}^{e}(\pi / N) \equiv V^{e} \cap f_{0}\left(V^{e}\right)
$$

Clearly, it consists of $N^{2}$ copies of $\mathbf{P}^{n-2}$, and it is the limit of the space $V_{b}^{e}$ as $\tau \uparrow$ $\pi / N$, cf. (4.129).

It is a remarkable fact that $V_{b}^{e}$ is actually $\tau$-independent for $N=3$ :

$$
V_{b}^{e}=V_{b}^{e}(\pi / N), \quad N=3
$$


Possibly, this is true for $N>3$ as well. The proof of (4.158) that we shall now sketch does not generalize to $N>3$, however. Let us put

$$
\begin{gathered}
C_{\nu} \equiv\left\{\left(z_{0}, z_{1}, z_{2}\right) \in \mathbf{P}^{2} \mid z_{\nu}=0\right\}, \quad \nu=0,1,2 \\
P_{\nu \rho} \equiv C_{\nu} \cap f_{0}\left(C_{\rho}\right)
\end{gathered}
$$

Using (4.128) it is routine to verify

$$
\begin{array}{lll}
P_{00}=(0,1,-1), & P_{01}=\left(0,1, e^{-i \pi / 3}\right), & P_{02}=\left(0,1, e^{i \pi / 3}\right) \\
P_{10}=(1,0,-1), & P_{11}=\left(1,0, e^{i \pi / 3}\right), & P_{12}=\left(1,0, e^{-i \pi / 3}\right) \\
P_{20}=(1,-1,0), & P_{21}=\left(1, e^{-i \pi / 3}, 0\right), & P_{22}=\left(1, e^{i \pi / 3}, 0\right)
\end{array}
$$

Then one easily checks

$$
\begin{gathered}
P_{00} \longrightarrow P_{00} \\
P_{01} \longrightarrow P_{20} \longrightarrow P_{02} \longrightarrow P_{10} \longrightarrow P_{01} \\
P_{11} \longrightarrow P_{21} \longrightarrow P_{22} \longrightarrow P_{12} \longrightarrow P_{11}
\end{gathered}
$$

where the arrows symbolize the map $f_{0}$.

To prove (4.158) it suffices to show

$$
C_{2} \cap V_{b}=\left\{P_{20}, P_{21}, P_{22}\right\}
$$

(Indeed, sufficiency follows from

$$
\begin{gathered}
\mathrm{g}\left(V_{b}\right)=\mathrm{g} \circ \phi\left(V^{e}\right)=\phi \circ \mathrm{g}_{q}\left(V^{e}\right)=\phi\left(V^{e}\right)=V_{b} \\
\mathrm{~g}\left(C_{2}\right)=C_{0}, \quad \mathrm{~g}^{2}\left(C_{2}\right)=C_{1}
\end{gathered}
$$

cf. (4.102), (4.114).) To verify (4.167) we first calculate the symmetric functions of the Lax matrix $L$ in the points $(1, w, 0), w \in \mathbf{C}^{*}$. (We need not consider $(1,0,0)$ and $(0,1,0)$ : these points are equal to $\phi\left(\varepsilon_{1}\right)$ and $\phi\left(\varepsilon_{2}\right)$, resp., and $\varepsilon_{\nu} \in V^{0}$.) From (1.97) and (4.70)-(4.73) we obtain

$$
S_{1}=\frac{w}{|w|}\left(\frac{\sin (\alpha-3 \tau / 2) \sin (\alpha+3 \tau / 2)}{\sin (\alpha-\tau / 2) \sin (\alpha+\tau / 2)}\right)^{1 / 2}, \quad S_{2}=\bar{S}_{1}, \quad S_{3}=1
$$

where 


$$
\alpha \equiv 3 \tau / 2+(\pi-3 \tau) /\left(1+|w|^{2}\right) \in(3 \tau / 2, \pi-3 \tau / 2)
$$

Next, we observe that $(1, w, 0)$ belongs to $V_{b}$ iff $L(1, w, 0)$ has a minimal spectral gap. In turn, this holds iff $\sigma(L)$ can be written

$$
\sigma(L)=\left\{-e^{i(\phi \pm \tau)}, e^{i(\phi+\psi)}\right\}, \quad \phi \in(-\pi, \pi], \quad \phi \in[-\pi+3 \tau, \pi-3 \tau]
$$

But if $\sigma(L)$ is of this form, one gets

$$
\left|S_{1}\right|=\left|2 \cos \tau-e^{i \psi}\right| \geq 2 \cos \tau-1
$$

with equality iff $\phi=0$. (Note $\cos \tau>1 / 2$ for $N=3$.) On the other hand, from (4.170) one readily deduces

$$
\left|S_{1}\right| \leq \frac{\cos (3 \tau / 2)}{\cos (\tau / 2)}=2 \cos \tau-1
$$

with equality iff $\alpha=\pi / 2$.

The upshot is, that if $L(1, w, 0)$ has a minimal spectral gap, then one must have $\phi=0$ in (4.172) and $|w|=1$ in (4.171). Using $S_{3}=1$, we then obtain three possible cases, viz.,

$$
\sigma(L)=\left\{-e^{ \pm i \tau}, 1\right\},\left\{e^{i(-\pi / 3 \pm \tau)},-e^{-i \pi / 3}\right\},\left\{e^{i(\pi / 3 \pm \tau)},-e^{i \pi / 3}\right\}
$$

It follows from the above that these cases actually occur iff $w=-1, e^{-i \pi / 3}, e^{i \pi / 3}$, yielding the points $P_{20}, P_{21}, P_{22}$, resp., cf. (4.163). Thus, (4.167) and (4.158) are now proved.

An interesting corollary of (4.158) is that (4.164)-(4.166) hold true under the action of $f$, too. In particular, one obtains a $\tau$-independent fixed point

$$
f(0,1,-1)=(0,1,-1)
$$

(To see this, one need only recall (4.129) and observe that $f$ leaves $V_{b}^{e}$ invariant.) Furthermore, it follows that one has

$$
\begin{gathered}
\phi\left(\boldsymbol{P}_{\nu \rho}\right)=\boldsymbol{P}_{\nu \rho}, \quad \nu \rho=00,12,21 \\
\phi\left(\boldsymbol{P}_{01}\right)=\boldsymbol{P}_{20}, \quad \phi\left(\boldsymbol{P}_{02}\right)=\boldsymbol{P}_{10}, \quad \phi\left(\boldsymbol{P}_{11}\right)=\boldsymbol{P}_{22}
\end{gathered}
$$

This state of affairs is depicted in Figure 4.

Finally, let us point out that one can directly verify that $P_{12}$ (say) is fixed under $\phi$. Indeed, using (1.93), (1.94) and (4.69)-(4.73) one obtains 


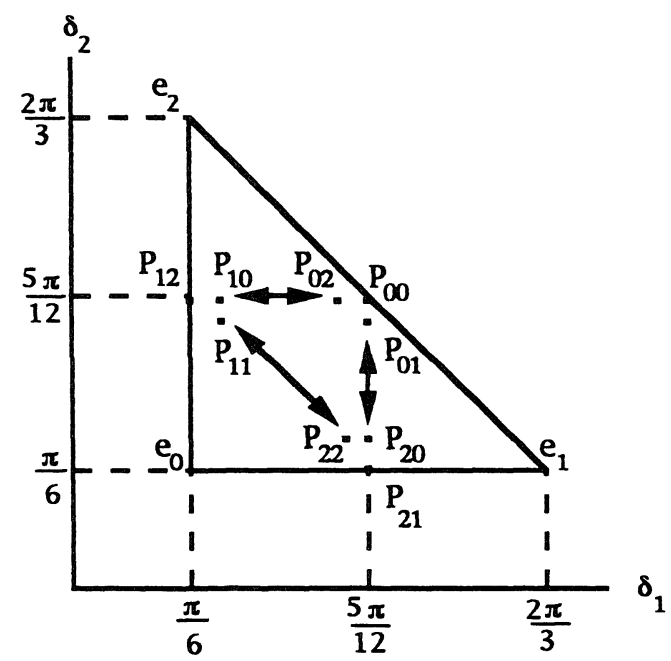

Figure 4. The action of the map $\phi$ on the points $P_{\nu \rho}$ for $\mu=i$ and $\tau=\pi / 6$. For clarity the non-fixed points are slightly displaced from the edge midpoints.

$$
\begin{gathered}
A\left(P_{12}\right)=\operatorname{diag}\left(e^{i(\pi / 3+\tau)}, e^{i(\pi / 3-\tau)},-e^{i \pi / 3}\right) \\
L\left(P_{12}\right)=\left(\begin{array}{ccc}
0 & e^{-i \pi / 3} \operatorname{sh}^{2} \rho & 2 \operatorname{ch} \rho \sin \tau / 2 \\
-1 & 0 & 0 \\
0 & -2 \operatorname{ch} \rho \sin \tau / 2 & e^{i \pi / 3} \operatorname{sh}^{2} \rho
\end{array}\right) \\
g\left(P_{12}\right)=\left(0, e^{-i(\pi / 6+\tau / 2)} \operatorname{sh} \rho \operatorname{ch} \rho, e^{-i \pi / 3} \operatorname{sh} \rho\right)
\end{gathered}
$$

where we have set

$$
\operatorname{sh} \rho \equiv(2 \cos \tau-1)^{1 / 2}
$$

A long, but straightforward calculation now yields

$$
U\left(P_{12}\right)=\frac{e^{i(\pi / 6+\tau)}}{2 \cos \tau / 2}\left(\begin{array}{ccc}
-i e^{i \tau / 2} & e^{i(\pi / 6-\tau / 2)} & e^{i \pi / 3} \operatorname{ch} \rho \\
e^{i(\pi / 6-\tau / 2)} & e^{i(5 \pi / 6+\tau / 2)} & \operatorname{ch} \rho \\
e^{i \pi / 3} \operatorname{ch} \rho & \operatorname{ch} \rho & 2 e^{-i \pi / 3} \sin \tau / 2
\end{array}\right)
$$

together with

$$
\left(U^{*} A U\right)\left(P_{12}\right)=L\left(P_{12}\right)^{t}, \quad\left(U^{*} L U\right)\left(P_{12}\right)=A\left(P_{12}\right)
$$

Thus one gets $\phi\left(P_{12}\right)=P_{12}$, as advertised. Note that $U\left(P_{12}\right)$ is symmetric, as 
should be the case on the fixed-point space of $\phi$, cf. (2.137) ; note also that one can now reobtain (4.177), (4.178) and (4.164)-(4.166) by using the maps $p, g, g_{q}$ and $\mathrm{k}$.

\section{Dynamics and Scattering}

\subsection{The Cases $\mathrm{III}_{\mathrm{nr}}$ and $\hat{\mathrm{II}}_{\mathrm{nr}}$}

The above construction of the action-angle maps $\tilde{\Phi}$ and $\Phi$ and their harmonic oscillator extensions $\tilde{\Phi}^{\#}$ and $\Phi^{\#}$ has not involved any Hamiltonian. On the other hand, the construction does make essential use of the Lax matrix (2.1) and the dual Lax matrix (2.24), and these matrices can be used as generating functions for a large collection of commuting Hamiltonians. We begin this section by studying Hamiltonians on $\tilde{\Omega} \simeq \mathbf{R}^{2} \times M$ defined by

$$
H_{h} \equiv \operatorname{Tr} h(L), \quad h \in \mathscr{C}
$$

Here, $\mathscr{C}$ denotes the class of non-constant entire functions of the form $\sum_{k=0}^{\infty} r_{k} x^{k}$, $r_{k} \in \mathbf{R}$. (Since $L$ is self-adjoint, we could just as well allow all $C_{\mathbf{R}}^{\infty}(\mathbf{R})$-functions, but $\mathscr{C}$ is large enough for our purposes.) Note that the choice $h(x)=x^{2} / 2$ yields the Sutherland Hamiltonian (2.2).

To study these dynamics (and the dual dynamics to be defined later on) we shall exploit the relations

$$
L(P) \sim \hat{L}(\hat{P}), \quad A(P) \sim \hat{A}(\hat{P}), \quad P \in \tilde{\Omega}, \quad \hat{P} \equiv \tilde{\Phi}^{\#}(P) \in \hat{\Omega}^{\# c}
$$

where $\sim$ denotes similarity. First, we claim that the flows $\exp \left(t H_{h}\right), h \in \mathscr{C}$, are complete and commute. To prove this, we set

$$
\hat{H}_{h} \equiv H_{h} \circ \tilde{\mathscr{E}}^{\#}
$$

and use (5.2) to obtain

$$
\hat{H}_{h}\left(\hat{\delta}_{s}, \hat{\gamma}_{s} ; u, v\right)=\sum_{j=1}^{N} h\left(\hat{\theta}_{j}\right)
$$

where (cf. (1.50), (1.73))

$$
\hat{\theta}_{j}=\hat{\delta}_{s}+\frac{|\mu g|}{2}(N+1-2 j)+\frac{|\mu|}{2}\left(\sum_{k=j}^{n}\left(u_{k}^{2}+v_{k}^{2}\right)-\frac{1}{N} \sum_{k=1}^{n} k\left(u_{k}^{2}+v_{k}^{2}\right)\right)
$$

Thus, $\hat{H}_{h}$ generates the flow 


$$
\begin{gathered}
\exp \left(t \hat{H}_{h}\right)\left(\hat{\delta}_{s}, \hat{\gamma}_{s} ; u, v\right)=\left(\hat{\delta}_{s}, \hat{\gamma}_{s}+\frac{t}{N} \sum_{j=1}^{N} h^{\prime}\left(\hat{\theta}_{j}\right) ; u_{1} \cos t \omega_{h, 1}+v_{1} \sin t \omega_{h, 1}, \ldots\right. \\
\left.\ldots, v_{n} \cos t \omega_{h, n}-u_{n} \sin t \omega_{h, n}\right)
\end{gathered}
$$

where the oscillation frequencies are given by

$$
\omega_{h, l} \equiv|\mu|\left(\sum_{j=1}^{l} h^{\prime}\left(\hat{\theta}_{j}\right)-\frac{l}{N} \sum_{j=1}^{N} h^{\prime}\left(\hat{\theta}_{j}\right)\right), \quad l=1, \ldots, n
$$

This flow is manifestly complete, and letting $h$ vary over $\mathscr{C}$ clearly yields commuting flows. Thus, the above claim now follows from Corollary 4.2.

We continue by noting that the above can be used to obtain complete and commuting flows on the reduced phase space $M$. Specifically, we may take $\delta_{s}, \gamma_{s}=$ 0 in (5.1) and view the resulting functions as Hamiltonians on $M$ (again denoted $H_{h}$ ). In view of the product structure of $\tilde{\Phi}^{\#}$ (cf. (1.74)) we can then use the relations

$$
L(m) \sim \hat{L}(\hat{m}), \quad A(m) \sim \hat{A}(\hat{m}), \quad m \in M, \quad \hat{m} \equiv \phi(m) \in \hat{M}
$$

to obtain

$$
\left(H_{h} \circ \mathrm{b}\right)(u, v)=\sum_{j=1}^{N} h\left(\hat{\theta}_{j}\right)
$$

where $\hat{\theta}_{j}$ is given by $(5.5)$ with $\hat{\delta}_{s} \equiv 0$. The corresponding flow on $\hat{M}$ is then given by (5.6) with the first two coordinates omitted.

In the Sutherland case $h(x)=x^{2} / 2$ the flow just defined actually arises quite naturally : it describes the center of mass frame motion. More precisely, the flow (5.6) leaves the submanifold $\{(0,0)\} \times \hat{M} \simeq \hat{M}$ invariant and coincides with the flow on $\hat{M}$ just detailed. Thus, this holds true for the Sutherland flow $\exp (t \tilde{H})$ on $\{(0,0)\} \times M$, too. Note that the center of mass frequencies

$$
\omega_{l}=|\mu| \sum_{j=1}^{l} \hat{\theta}_{j}, \quad \hat{\theta} \in A_{N}^{c l}, \quad \sum_{j=1}^{N} \hat{\theta}_{j}=0
$$

are rationally independent except on a set of measure zero. Thus the orbit closure is generically $n$-dimensional. As a consequence, the commutant of the reduced Sutherland Hamiltonian is abelian.

Obviously, the point $(0,0) \in \hat{M}$ is left invariant by all of the reduced flows $\exp \left(t \hat{H}_{h}\right)$. More generally, the equilibria for a fixed $h \in \mathscr{C}$ are the points in the set

$$
\hat{\boldsymbol{e}}_{h} \equiv\left\{(u, v) \in \mathbf{R}^{2 n} \mid u_{j}^{2}+v_{j}^{2} \neq 0 \Longrightarrow \omega_{h, j}(u, v)=0, j=1, \ldots, n\right\}
$$


In the Sutherland case all of the frequencies are positive on all of $\hat{M}$ (cf. (5.10)), so that

$$
\hat{e}_{h}=\{(0,0)\}, \quad h(x)=x^{2} / 2
$$

For other dynamics, however, $\hat{e}_{h}$ in general contains tori $\mathrm{T}^{j}, j \in\{1, \ldots, n\}$.

Returning to the $\hat{H}_{h}$ flow on $\mathbf{R}^{2} \times \hat{M}$, the equilibrium set is given by

$$
\begin{gathered}
\hat{E}_{h} \equiv\left\{\left(\hat{\delta}_{s}, \hat{\gamma}_{s} ; u, v\right) \in \mathbb{R}^{2} \times \hat{M} \mid \sum_{j=1}^{N} h^{\prime}\left(\hat{\theta}_{j}\right)=0, \quad u_{j}^{2}+v_{j}^{2} \neq 0 \Longrightarrow\right. \\
\left.\omega_{h, j}(\hat{\theta})=0, \quad j=1, \ldots, n\right\}
\end{gathered}
$$

In particular, this yields

$$
\hat{E}_{h}=\{(0, a ; 0,0) \mid a \in \mathbf{R}\}, \quad h(x)=x^{2} / 2
$$

in the Sutherland case. Thus the points (1.109) are the only equilibria of the $\tilde{H}$ flow, as announced. (Recall (4.33).) It readily follows that $\tilde{H}$ has a global minimum at the points $\left(q_{a}^{e}, 0\right)$ and no further critical points. (The vanishing of $\nabla \tilde{H}$ at $\left(q_{a}^{e}, 0\right)$ can of course be seen directly, cf. (1.1).)

It is not hard to verify that these properties hold true more generally for the dynamics

$$
H_{k} \equiv \operatorname{Tr} h_{k}(L), \quad h_{k}(x) \equiv \frac{1}{2 k} x^{2 k}, \quad k=1,2,3, \ldots
$$

Specifically, $H_{k}$ has a global minimum

$$
H_{k}\left(\boldsymbol{q}_{a}^{e}, 0\right)=\frac{1}{2 k} \sum_{j=1}^{N}\left(\frac{|\mu g|}{2}(N+1-2 j)\right)^{2 k}
$$

and no further critical points. (Observe that $\omega_{h_{k}, l}>0$ whenever $\sum_{j=1}^{N} \hat{\theta}_{j}^{2 k-1}=0$.)

Next, we derive a representation for the position part $q(t)$ of the flows $\exp \left(t H_{h}\right)$. This involves the matrix-valued function on $\mathbf{R} \times \tilde{\Omega}$ given by

$$
A_{h}(t, P) \equiv A(P) \exp \left(t \mu h^{\prime}(L(P))\right)
$$

Theorem 5.1. Let $h \in \mathscr{C}, t \in \mathbb{R}$ and $P \in \tilde{\Omega}$. Then the matrix $A_{h}(t, P)$ has simple spectrum on the unit circle. Its eigenvalues $\alpha_{1}(t), \ldots, \alpha_{N}(t)$ can be ordered such that the position part of the integral curve $\exp \left(t H_{h}\right)(P)$ is given by

$$
q_{j}(t)=\mu^{-1} \ln \left(\alpha_{j}(t)\right), \quad j=1, \ldots, N
$$

Proof. This follows from the chain of similarities 
$A_{h}(t, P) \sim \hat{A}(\hat{P}) \exp \left(t \mu h^{\prime}(\hat{L}(\hat{P}))\right) \sim \hat{A}\left(\exp \left(t \hat{H}_{h}\right)(\hat{P})\right) \sim A\left(\exp \left(t H_{h}\right)(P)\right)$

cf. (5.2). (The second step is most easily verified first on $\hat{\Omega}^{c}$, using the coordinates (1.50), (1.64); its validity on $\hat{\Omega}^{\# c}$ then follows by continuity.)

Combining the equilibrium property

$$
\exp \left(t H_{k}\right)\left(q^{e}, 0\right)=\left(q^{e}, 0\right), \quad \forall(t, k) \in \mathbf{R} \times\{1,2, \ldots\}
$$

and (5.19), we obtain an isospectrality relation that is quite non-obvious, viz.,

$$
\begin{aligned}
& \sigma\left(\operatorname{diag}\left(\omega^{n}, \omega^{n-2}, \ldots, \omega^{-n}\right) \exp \left(i r E_{1}^{m}\right)\right) \\
& \quad=\left\{\omega^{n}, \omega^{n-2}, \ldots, \omega^{-n}\right\}, \quad \forall(r, m) \in \mathbf{R} \times\{1,3,5, \ldots\}
\end{aligned}
$$

with $E_{1}$ given by (A.31).

We proceed by examining partition functions for the Hamiltonians (5.15). In keeping with the physical picture sketched in Section 1.1, these make no sense on $\tilde{\Omega}$ (due to 'infinite volume divergence'). However, the Hamiltonians are invariant under the $\mathbf{Z}$-action (1.12) in view of (2.44). Thus they descend to smooth Hamiltonians (again denoted $H_{k}$ ) on $\Omega$, cf. the diagram (1.74). Moreover, it is clear that the corresponding flows on $\Omega$ are complete and commute. Since one is now dealing with particles on a ring, one expects that the partition functions (cf. (1.14))

$$
Z_{k}(T) \equiv \int_{\Omega} \exp \left(-H_{k}(x, p) / T\right) d x d p, \quad T \in(0, \infty), \quad k=1,2, \ldots
$$

are finite. This expectation is borne out by the following result, which expresses $Z_{k}$ in a far simpler form.

Theorem 5.2. One has

$$
Z_{k}(T)=\left(\frac{2 \pi}{|\mu|}\right)^{N} \int_{A_{N}} \exp \left(-\frac{1}{2 k T} \sum_{j=1}^{N} \hat{p}_{j}^{2 k}\right) d \hat{p}, \quad k=1,2, \ldots
$$

where $A_{N}$ is defined by (1.49).

Proof. We may restrict the integration in (5.22) to $\Omega_{r}$, since $\Omega \backslash \Omega_{r}$ has measure zero. Now we use the action-angle map $\Phi: \Omega, \longrightarrow \hat{\Omega},(x, p) \longmapsto(\hat{x}, \hat{p})$ (cf. (1.67), (1.68)) to change variables. Since $\Phi$ is canonical, the Jacobian equals 1 , so that (5.23) follows from (1.48) and (5.15). 
We continue by studying a collection of dual dynamics defined on the extended dual phase space $\hat{\Omega}^{\# c} \equiv \mathbf{R}^{2} \times \hat{M}$, cf. the diagram (1.74). These are given by

$$
D_{h} \equiv \operatorname{Tr}(h(\hat{A})+\text { h.c. }), \quad h \in \mathscr{C}_{e}
$$

Here, $\mathscr{C}_{e}$ is the class of all non-constant entire functions and h.c. stands for hermitean conjugate. (The dual Hamiltonian (1.106), (1.107) is obtained by choosing $h(x)=x / 2$.) Note that $D_{h}$ is a $C^{\omega}$ function on $\mathbf{R}^{2} \times \hat{M}$, since $\hat{A}$ is.

We claim that all of the flows $\exp \left(t D_{h}\right)$ are complete and commute. Indeed, setting

$$
\check{D}_{h} \equiv D_{h} \circ \tilde{\Phi}^{\#}
$$

we have

$$
\check{D}_{h}(q, \theta)=\sum_{j=1}^{N}\left(h\left(\exp \mu q_{j}\right)+\text { c.c. }\right)
$$

Thus, on $\tilde{\Omega}$ we get the complete commuting flows

$$
\exp \left(t \check{D}_{h}\right)(q, \theta)=\left(q_{1}, \ldots, q_{N}, \theta_{1}+t v_{h, 1}, \ldots, \theta+t v_{h, N}\right)
$$

where

$$
v_{h, j} \equiv-\mu\left(\exp \left(\mu q_{j}\right) h^{\prime}\left(\exp \mu q_{j}\right)-\text { c.c. }\right)
$$

Now Corollary 4.2 entails that the flows $\exp \left(t D_{h}\right)$ on $\mathbf{R}^{2} \times \hat{M}$ are complete and commute. Using (5.8) as before, we can also obtain complete and commuting flows on the reduced phase space $\hat{M}$.

To obtain the analog of Theorem 5.1 we introduce

$$
\hat{L}_{h}(t, \hat{P}) \equiv \hat{L}(\hat{P})-t \mu\left(\hat{A}(\hat{P}) h^{\prime}(\hat{A}(\hat{P}))-\text { h.c. }\right)
$$

Theorem 5.3. Let $h \in \mathscr{C}_{e}, t \in \mathbf{R}$, and $\hat{P} \in \hat{\Omega}^{\# c}$. Then the matrix $\hat{L}_{h}(t, \hat{P})$ has simple and real spectrum. With the eigenvalue ordering $\lambda_{1}(t)>\cdots>\lambda_{N}(t)$, one has

$$
\lambda_{j}(t)=\hat{\theta}_{j}(t)
$$

where $\hat{\theta}_{j}(t)$ is given by the integral curve $\exp \left(t D_{h}\right)(\hat{P})$ and $(5.5)$.

Proof. We have

$$
\hat{L}_{h}(t, \hat{P}) \sim L(P)-t \mu\left(A(P) h^{\prime}(A(P))-\text { h.c. }\right) \sim L\left(\exp \left(t \check{D}_{h}\right)(P)\right)
$$




$$
\sim \hat{L}\left(\exp \left(t D_{h}\right)(\hat{P})\right)
$$

where the second step follows from (5.27), (5.28) and (2.1). Recalling (2.5), the assertions readily follow.

Next, we use this result to study the long-time asymptotics of the quantities $\hat{\theta}_{j}(t)$. Fixing $\hat{\boldsymbol{P}} \in \mathbf{R}^{2} \times \hat{M}$, we set $(q, \theta) \equiv \tilde{\mathscr{E}}^{\#}(\hat{\boldsymbol{P}})$ and introduce the distinct-velocity subset

$$
\left(\mathbf{R}^{2} \times \hat{\boldsymbol{M}}\right)_{h, \neq} \equiv\left\{\hat{\boldsymbol{P}} \in \mathbf{R}^{2} \times \hat{\boldsymbol{M}} \mid v_{h, 1}, \ldots, v_{h, N} \operatorname{distinct}\right\}
$$

This is an open dense full measure submanifold, since $h$ is a non-constant entire function.

Theorem 5.4. Let $h \in \mathscr{C}_{e}$ and $\hat{P} \in\left(\mathbf{R}^{2} \times \hat{M}\right)_{h, \neq}$, and let $\sigma \in S_{N}$ be such that

$$
v_{h, \sigma(1)}>\cdots>v_{h, \sigma(N)}
$$

Then one has

$$
\hat{\theta}_{N-j+1}^{j}(t)-\theta_{\sigma(j)}-t v_{h, \sigma(j)} \rightarrow 0, \quad t \rightarrow \pm \infty, \quad j=1, \ldots, N
$$

Proof. In view of Theorem 5.3 we need only determine the spectral asymptotics of

$$
L(P)+t \operatorname{diag}\left(v_{h, 1}(P), \ldots, v_{h, N}(P)\right)
$$

This can be read off from Theorem Al in I, yielding (5.34).

To conclude, we consider the exceptional set

$$
\hat{\Omega}^{\# c} \backslash \hat{\Omega}^{c}=\mathbf{R}^{2} \times \hat{M}^{e}
$$

cf. (4.79). As we have seen above, this set is characterized by at least one of the differences $\hat{\theta}_{j}-\hat{\theta}_{j+1}$ being equal to $|\mu g|$. Fixing $h \in \mathscr{C}_{e}$ and $\hat{P} \in \mathbf{R}^{2} \times \hat{M}$, the orbit $\exp \left(t D_{h}\right)(\hat{P})$ either belongs to $\mathbf{R}^{2} \times \hat{M}^{e}$ or meets $\mathbf{R}^{2} \times \hat{M}^{e}$ for a discrete set $C_{h, \hat{P}}$ of times. In view of Theorem 5.4 the latter possibility applies (with $\left|C_{h, \hat{P}}\right| \in \mathbf{N}$ ) whenever $\hat{P}$ belongs to $\left(\mathbf{R}^{2} \times \hat{M}\right)_{h, \neq}$. Now the phase factors $\exp \left(\mu q_{j}(\hat{P})\right)$ are distinct, so one can find $h \in \mathscr{C}_{e}$ such that the velocities $v_{h, 1}(\hat{\boldsymbol{P}}), \ldots, v_{h, N}(\hat{\boldsymbol{P}})$ are distinct. As a consequence, there exists no $\hat{P} \in \mathbf{R}^{2} \times \hat{M}^{e}$ for which the orbits $\exp \left(t D_{h}\right)(\hat{P}), t \in \mathbf{R}$, belong to $\mathbf{R}^{2} \times \hat{M}^{e}$ for all $h \in \mathscr{C}_{e}$; equivalently, for any $\hat{\boldsymbol{P}} \in \mathbf{R}^{2}$ $\times \hat{\boldsymbol{M}}^{e}$ one can find $\hat{\boldsymbol{P}}^{0} \in \mathbf{R}^{2} \times \hat{\boldsymbol{M}}^{0}, h \in \mathscr{C}_{e}$ and $t \in \mathbf{R}$ such that $\hat{\boldsymbol{P}}=\exp \left(t D_{h}\right)\left(\hat{\boldsymbol{P}}^{0}\right)$. 
Consequently, the extension $\mathbb{R}^{2} \times \hat{M}$ of $\mathbf{R}^{2} \times \hat{M}^{0}$ is minimal in regard to completing all of the orbits $\exp \left(t D_{h}\right)\left(\hat{P}^{0}\right), \hat{P}^{0} \in \mathbf{R}^{2} \times \hat{M}^{0}$, as defined piecewisenamely, for $t \in \mathbf{R} \backslash C_{h, \hat{P}^{0}}$-via the map $\tilde{\mathscr{E}}$. More precisely, adapting the minimal extension procedure introduced in II (cf. the paragraph containing (6.140)), the $\operatorname{map} Q \longmapsto \hat{P}=\exp \left(t D_{h}\right)\left(\hat{P}^{0}\right)$ between equivalence classes $Q$ of triples $\left(\hat{P}^{0}, h, t\right), \hat{P}^{0}$ $\in \mathbf{R}^{2} \times \hat{M}^{0}, h \in \mathscr{C}_{e}, t \in \mathbf{R}$, and points $\hat{P} \in \mathbf{R}^{2} \times \hat{M}$ is a well-defined bijection.

Of course, these considerations apply with obvious changes to the various reduced flows and phase spaces.

\subsection{The Cases $\mathrm{III}_{\text {rel }}$ and $\hat{\mathrm{III}}_{\text {rel }}$}

We proceed along the same lines as in the previous section. First, we consider a class of dynamics on $\tilde{\Omega}$ defined by

$$
H_{h} \equiv \operatorname{Tr} h\left(\beta^{-1} \ln L\right), \quad h \in \mathscr{C}
$$

(Recall $L$ is positive in this case ; thus, its logarithm can be defined in the obvious way.) Clearly, the Hamiltonian (2.52) is obtained by choosing $h(x)=\operatorname{ch} \beta x$.

Defining $\hat{H}_{h}$ by (5.3) (with $\tilde{\mathscr{E}}^{\#}$ the $\mathrm{III}_{\text {rel }}$ map, of course), we infer from (5.2) and (2.56) that (5.4)-(5.7) still hold true. Using Corollary 4.5 we then deduce that all flows $\exp \left(t H_{h}\right), h \in \mathscr{C}$, are complete and commute.

As before, we obtain commuting complete flows on $M$ by taking $\delta_{s}, \gamma_{s}=0$ in (5.1). The point $b(0,0)$ is an equilibrium for all of the flows, and more generally the set of equilibria is given by $\mathrm{b}\left(\hat{e}_{h}\right)$ with $\hat{e}_{h}$ defined by (5.11). Similarly, the $H_{h}$ flow on $\tilde{\Omega}$ has its equilibria at the points of $\tilde{\mathscr{E}}^{\#}\left(\hat{E}_{h}\right)$, with $\hat{E}_{h}$ given by (5.13).

Next, consider the dynamics

$$
H_{k} \equiv \frac{1}{2} \operatorname{Tr}\left(L^{k}+L^{-k}\right), \quad k=1,2, \ldots
$$

which correspond to the functions $h_{k}(x)=\operatorname{ch} \beta k x$. Since (4.33) and (4.30) still hold, we obtain in the same way as before

$$
\begin{gathered}
\exp \left(t H_{k}\right)\left(q_{a}^{e}, 0\right)=\left(q_{a}^{e}, 0\right) \\
H_{k}\left(q_{a}^{e}, 0\right)=\sum_{j=1}^{N} \operatorname{ch}(k z(N+1-2 j))
\end{gathered}
$$

Moreover, it follows again that no other equilibria occur ; correspondingly, $H_{k}$ has a global minimum at $\left(q_{a}^{e}, 0\right)$ and no other critical points.

The analog of Theorem 5.1 involves the matrix

$$
A_{h}(t, P) \equiv A(P) \exp \left(t \mu h^{\prime}\left(\beta^{-1} \ln L(P)\right)\right)
$$


Theorem 5.5. The assertions of Theorem 5.1 hold true in the $\mathrm{II}_{\text {rel }}$ case, too.

Proof. Substituting $\hat{L}(\hat{P}) \rightarrow \beta^{-1} \ln \hat{L}(\hat{P})$ in (5.19), the proof of Theorem 5.1 applies verbatim.

As the analog of (5.21) we obtain the remarkable isospectrality relation $\sigma\left(\operatorname{diag}\left(\omega^{n}, \ldots, \omega^{-n}\right) \exp \left(\operatorname{ir}\left(E^{k}-E^{-k}\right)\right)=\left\{\omega^{n}, \ldots, \omega^{-n}\right\}, \quad \forall(r, k) \in \mathbf{R} \times \mathbf{N}\right.$

(Recall (4.55), (A.29).)

Next, we consider the partition functions (5.22).

Theorem 5.6. One has

$$
Z_{k}(T)=\left(\frac{2 \pi}{|\mu|}\right)^{N} \int_{A_{N}} \exp \left(-\sum_{j=1}^{N} \operatorname{ch}\left(\beta k \hat{p}_{j}\right) / T\right) d \hat{p}, \quad k=1,2, \ldots
$$

Proof. This follows in the same way as (5.23).

By means of (5.24) we obtain once more a class of real-analytic dual Hamiltonians on $\mathbf{R}^{2} \times \hat{M}$. Defining $\check{D}_{h}$ by (5.25), we deduce again (5.26)-(5.28), so Corollary 4.5 yields completeness and commutativity of the flows $\exp \left(t D_{h}\right)$.

Instead of (5.29) we now need the matrix

$$
\hat{L}_{h}(t, \hat{P}) \equiv \hat{L}(\hat{P}) \exp \left(-t \mu\left(\hat{A}(\hat{P}) h^{\prime}(\hat{A}(\hat{P}))-\text { h.c. }\right)\right)
$$

Theorem 5.7. Let $h \in \mathscr{C}_{e}, t \in \mathbf{R}$, and $\hat{P} \in \hat{\Omega}^{\# c}$. Then the matrix $\hat{L}_{h}(t, \hat{P})$ has simple and positive spectrum. With the eigenvalue ordering $\lambda_{1}(t)>\cdots>\lambda_{N}(t)$, one has

$$
\beta^{-1} \ln \left(\lambda_{j}(t)\right)=\hat{\theta}_{j}(t)
$$

Proof. We have

$$
\begin{aligned}
\hat{L}_{h}(t, \hat{P}) & \sim L(P) \exp \left(-t \mu\left(A(P) h^{\prime}(A(P))-\text { h.c. }\right)\right) \sim L\left(\exp \left(t \check{D}_{h}\right)(P)\right) \\
& \sim \hat{L}\left(\exp \left(t D_{h}\right)(\hat{P})\right)
\end{aligned}
$$

where the second step follows from (5.27), (5.28) and (2.51). Thus, the assertions follow from (2.56). 
With the definition (5.32) in effect, we are prepared for the next result.

Theorem 5.8. Fixing $h \in \mathscr{C}_{e}, \hat{P} \in\left(\mathbf{R}^{2} \times \hat{M}\right)_{h, \neq}$ and $\sigma \in S_{N}$ such that (5.33) holds, one has

$$
\begin{gathered}
\hat{\theta}_{N-j+1}^{j}(t)-\theta_{\sigma(j)} \pm \frac{1}{2}\left(\sum_{k<J}-\sum_{k>J}\right) \delta\left(q_{\sigma(j)}-q_{\sigma(k)}\right)-t v_{h, \sigma(j)} \rightarrow 0, \\
t \rightarrow \pm \infty, \quad j=1, \ldots, N
\end{gathered}
$$

where

$$
\delta(q) \equiv \beta^{-1} \ln \left(1+\frac{\operatorname{sh}^{2} z}{\sin ^{2} \frac{|\mu|}{2} q}\right)
$$

Proof. By virtue of Theorem 5.7 we need only calculate the spectral asymptotics of

$$
L(P) \exp \left(t \operatorname{diag}\left(v_{h, 1}(\boldsymbol{P}), \ldots, v_{h, N}(\boldsymbol{P})\right)\right.
$$

Combining Theorem A2 in I (or an obvious specialization of Theorem C1 in II) and Cauchy's identity (A.2) one readily obtains the above result.

To conclude this section, we observe that the discussion below Theorem 5.4 also applies to the case in hand, with Theorem 5.8 playing the part of Theorem 5.4.

\subsection{The Case $\mathrm{II}_{\mathrm{b}}$}

In this section we study the set of $C^{\omega}$ Hamiltonians on $\Omega^{\# c}=\mathbb{R}^{2} \times M$ defined by

$$
H_{h}=\operatorname{Tr}(h(L)+\text { h.c. }), \quad h \in \mathscr{C}_{e}
$$

More precisely, via (2.82) the rhs yields a function on $\Omega^{c}=\mathbb{R}^{2} \times M^{0}$, and this function has a $C^{\omega}$ extension to $\Omega^{\# c}$ (since $L$ has, cf. Section 4.3). Using (2.125), (4.101) and (2.82), we deduce that all of these Hamiltonians are invariant under the $\mathbb{Z} \times \mathbf{Z}$-action on $\Omega^{\# c}$, so they descend to smooth Hamiltonians (again denoted $H_{h}$ ) on $\Omega^{\#}$, cf. the diagram (1.102). The Hamiltonian (1.4) and its cover $H^{c}$ arise by taking $h(x)=x / 2$ in (5.50).

We proceed by studying the above dynamics along the same lines as in the two preceding sections. The role of (5.2) is now played by 


$$
L(P) \sim \hat{L}(\hat{\boldsymbol{P}}), \quad A(P) \sim \hat{A}(\hat{\boldsymbol{P}}), \quad P \in \Omega^{\# c}, \quad \hat{P} \equiv \tilde{\Phi}^{\times}(P) \in \hat{\Omega}^{\# c}
$$

First, setting

$$
\hat{H}_{h} \equiv H_{h} \circ \tilde{\mathscr{E}}^{\times}
$$

we obtain

$$
\hat{H}_{h}\left(\hat{\delta}_{s}, \hat{\gamma}_{s} ; \hat{z}_{0}, \ldots, \hat{z}_{n}\right)=\sum_{j=1}^{N}\left(h\left(\exp \beta \hat{\theta}_{j}\right)+\text { c.c. }\right)
$$

where (cf. (1.50), (1.93)-(1.99))

$$
\begin{aligned}
\hat{\theta}_{j} & =\hat{\delta}_{s}+\frac{|\mu g|}{2}(N+1-2 j)+\frac{2|\mu| R^{2}}{(\hat{z}, \hat{z})}\left(\sum_{k=j}^{n}\left|\hat{z}_{k}\right|^{2}-\frac{1}{N} \sum_{k=1}^{n} k\left|\hat{z}_{k}\right|^{2}\right), \\
R^{2} & \equiv \frac{1}{|\beta \mu|}(\pi-N \tau)
\end{aligned}
$$

The corresponding Hamiltonian flows read

$$
\begin{aligned}
& \exp \left(t \hat{H}_{h}\right)\left(\hat{\delta}_{s}, \hat{\gamma}_{s} ; \hat{z}_{0}, \ldots, \hat{z}_{n}\right) \\
& \quad=\left(\hat{\delta}_{s}, \hat{\gamma}_{s}+\frac{t}{N} \sum_{j=1}^{N} v_{h, j} ; \hat{z}_{0}, \hat{z}_{1} \exp \left(i t \omega_{h, 1}\right), \ldots, \hat{z}_{n} \exp \left(i t \omega_{h, n}\right)\right)
\end{aligned}
$$

where

$$
\begin{gathered}
\omega_{h, l} \equiv|\mu|\left(\sum_{j=1}^{l} v_{h, j}-\frac{l}{N} \sum_{j=1}^{N} v_{h, j}\right), \quad l=1, \ldots, n \\
v_{h, j} \equiv \beta\left(\exp \left(\beta \hat{\theta}_{j}\right) h^{\prime}\left(\exp \beta \hat{\theta}_{j}\right)-\text { c.c. }\right), \quad j=1, \ldots, N
\end{gathered}
$$

(Use (1.64), (1.93) to check this.)

Using Corollary 4.7 we now deduce that all of the flows $\exp \left(t H_{h}\right), h \in \mathscr{C}_{e}$, are complete and commute. As before, we can obtain complete commuting flows on $M$ $\simeq \mathbf{P}^{n}$ by omitting the first two coordinates. (Of course, in this case completeness already follows from compactness of $M$.)

We proceed by examining special orbits, considering first the reduced flows on M. Using (the reduced version of ) (5.55) and (4.77), we begin by noting

$$
\exp \left(t H_{h}\right)\left(\varepsilon_{\nu}\right)=\varepsilon_{\nu}, \quad \forall(t, h, \nu) \in \mathbf{R} \times \mathscr{C}_{e} \times\{0, \ldots, n\}
$$

That is, all of the flows have equilibria at the points $\varepsilon_{0}, \ldots, \varepsilon_{n}$. These $N$ equilibria 
need not be the only ones, however. The generalization of the equilibrium set (5.11) reads

$$
\hat{e}_{h}=\left\{\hat{z} \in \hat{M} \mid \text { if } \hat{z}_{\nu_{1}} \hat{z}_{\nu_{2}} \neq 0, \quad \text { then } \omega_{h, \nu_{1}}(\hat{z})=\omega_{h, \nu_{2}}(\hat{z})\right\}, \quad \omega_{h, 0} \equiv 0
$$

cf. (5.55). Since the frequencies depend only on $\left|\hat{z}_{0}\right|, \ldots,\left|\hat{z}_{n}\right|$, additional equilibria belong to tori $\mathbb{T}^{j}, j \in\{1, \ldots, n\}$. (Kreis)

It is instructive to look at an explicit example for $n=2$. Consider the circle

$$
\hat{K} \equiv\left\{\left(0,1, e^{i t}\right) \mid t \in[0,2 \pi)\right\} \subset \mathbb{P}^{2}
$$

From (5.54) we see that all points of $\hat{K}$ yield

$$
\hat{\theta}_{3}= \pm \frac{1}{|\beta|}(\pi-\tau), \quad \hat{\theta}_{2}=0
$$

Using now (5.56), (5.57) we conclude that on $\hat{K}$

$$
v_{h, 1}=-v_{h, 3}, \quad v_{h, 2}=0, \quad \omega_{h, 1}=\omega_{h, 2}, \quad \forall h \in \mathscr{C}
$$

As a consequence, $\hat{K}$ consists of equilibria for the $\hat{H}_{h}$ flow whenever $h \in \mathscr{C} \subset \mathscr{C}_{e}$.

Next, we observe that $\hat{K}$ contains the points (4.161). From (4.177), (4.178) it then follows that for all $h \in \mathscr{C}$ the reduced $H_{h}$ flows have a circle $K=\mathrm{b}(\hat{K})$ of equilibria, connecting the points $P_{00}, P_{10}$ and $P_{20}$ (cf. Figure 4). However, for $h \in$ $\mathscr{C}_{e} \backslash \mathscr{C}$ this need not be true. For instance, taking $h(x)=i x / 2$, one gets on $\hat{K}$

$$
v_{h, 1}=v_{h, 3}=|\beta| \cos \tau, \quad v_{h, 2}=-|\beta|, \quad \omega_{h, 1} \neq \omega_{h, 2}
$$

which implies

$$
\left\{\exp \left(t H_{h}\right)\left(P_{00}\right) \mid h(x)=i x / 2, t \in \mathbf{R}\right\}=K
$$

Returning to the general $N$ case, we may use (5.53), (5.54) to infer

$$
H_{h}\left(\varepsilon_{\nu}\right)=\sum_{j=1}^{N}(h(\exp [i \tau(N+1-2 j)-2 \pi i \nu / N])+\text { c.c. })
$$

For $h(x)=x / 2$ this specializes to

$$
H^{c}\left(\varepsilon_{\nu}\right)=\cos (2 \pi \nu / N) \sin (N \tau) / \sin \tau, \quad \nu=0, \ldots, n
$$

It is not hard to see that $\nu=0$ yields the (global) maximum of $H^{c}$. Similarly, when $N$ is even, the choice $\nu=N / 2$ yields the minimum. 
We proceed by noting that the equilibrium set for the $H_{h}$ flow on $\Omega^{\# c}=\mathbf{R}^{2} \times$ $M$ is given by

$$
\begin{aligned}
E_{h} & =\tilde{E}^{\times}\left(\left\{\left(\hat{\delta}_{s}, \hat{\gamma}_{s} ; \hat{z}\right) \in \mathbf{R}^{2} \times \hat{M} \mid \sum_{j=1}^{N} v_{h, j}=0 \text {, if } \hat{z}_{\nu_{1}} \hat{z}_{\nu_{2}} \neq 0 \text {, then } \omega_{h, \nu_{1}}\left(\hat{\delta}_{s}, \hat{z}\right)\right.\right. \\
& \left.\left.=\omega_{h, \nu_{2}}\left(\hat{\delta}_{\mathrm{s}}, \hat{z}\right)\right\}\right)
\end{aligned}
$$

cf. (5.55). Next, we introduce the special Hamiltonians

$$
H_{k} \equiv \operatorname{Tr}\left(h_{k}(L)+\text { h.c. }\right), \quad h_{k}(x) \equiv \frac{1}{2} x^{k}, \quad k=1,2 . \ldots
$$

Using (2.130) one infers that all of these functions on $\Omega^{\# c}$ are $K$-invariant. Moreover, for all of them $E_{h}$ is easily seen to contain the points

$$
\left(a, \frac{2 \pi}{|\beta|}\left(\frac{\nu}{N}+\frac{l}{2}\right) ; \varepsilon_{\nu}\right), \quad \nu \in\{0, \ldots, n\}, \quad l \in \mathbf{Z}, \quad a \in \mathbf{R}
$$

and from (5.52)-(5.54) one obtains

$$
H_{k}\left(a, \frac{2 \pi}{|\beta|}\left(\frac{\nu}{N}+\frac{l}{2}\right) ; \varepsilon_{\nu}\right)=(-)^{l} \frac{\sin N k \tau}{\sin k \tau}, \quad \tau \notin \frac{\pi}{k} \mathbf{N}
$$

On the compact quotient manifold $\Omega^{\#}$ the above points give rise to two circles of critical points for the quotient Hamiltonians (again denoted $H_{k}$ ), cf. (4.102)(4.106). Specifically, one obtains

$$
\begin{aligned}
& K_{+}=\left\{\left(a, 0 ; \varepsilon_{0}\right) \mid a \in[0,2 \pi / N|\mu|)\right\} \quad(l \text { even }) \\
& K_{-}=\left\{\left(a, 0 ; \varepsilon_{N / 2}\right) \mid a \in[0,2 \pi / N|\mu|)\right\} \quad(l \text { odd, } N \text { even }) \\
& K_{-}=\left\{\left(a, \pi / N|\beta| ; \varepsilon_{(N+1) / 2}\right) \mid a \in[0,2 \pi / N|\mu|)\right\} \quad(l \text { odd, } N \text { odd })
\end{aligned}
$$

when the coordinates in (4.107) are used. Clearly, these circles belong to $\Omega$; w.r.t. the coordinates $(x, p)$ in (1.41) they can be written

$$
\begin{gathered}
K_{+}=\left\{\left(q^{e}+\alpha(1, \ldots, 1) ; 0\right) \mid \alpha \in\left(-\frac{\pi}{N|\mu|}, \frac{\pi}{N|\mu|}\right]\right\} \quad(l \text { even }) \\
K_{-}=\left\{\left(q^{e}+\alpha(1, \ldots, 1) ; \frac{\pi}{|\beta|}(1, \ldots, 1)\right) \mid \alpha \in\left(-\frac{\pi}{N|\mu|}, \frac{\pi}{N|\mu|}\right]\right\} \quad(l \text { odd })
\end{gathered}
$$


Of course, the quotient Hamiltonian $H_{1}$ equals the Hamiltonian (1.4) on $\Omega$. Note that $H_{1}$ attains its maximum/minimum on the circles $K_{+} / K_{-}$.

In order to present the analog of Theorem 5.1, we introduce the matrix-valued function on $\mathbf{R} \times \Omega^{\# c}$

$$
A_{h}(t, P) \equiv A(P) \exp \left(t \mu\left(L(P) h^{\prime}(L(P))-\text { h.c. }\right)\right)
$$

and the vector-valued function on $\Omega^{\# c}$

$$
\begin{aligned}
q_{j}(P) & =\delta_{s}+\frac{1}{|\mu|}\left((N+1-2 j) \tau+\frac{2(\pi-N \tau)}{(z, z)}\left(\sum_{k=j}^{n}\left|z_{k}\right|^{2}-\frac{1}{N} \sum_{k=1}^{n} k\left|z_{k}\right|^{2}\right)\right), \\
j & =1, \ldots, N
\end{aligned}
$$

In contrast to previous cases, $P$ varies over a phase space that is not equal to the cotangent bundle of a configuration space. Even so, it is natural to refer to $q(P)$ as the position part of $P$.

Theorem 5.9. With $\mathscr{C}$ and $\Omega$ replaced by $\mathscr{C}_{e}$ and $\Omega^{\# c}$, the assertions of Theorem 5.1 hold true in the $\mathrm{III}_{\mathrm{b}}$ case.

Proof. As before, this follows from

$$
\begin{aligned}
A_{h}(t, P) & \sim \hat{A}(\hat{P}) \exp \left(t \mu\left(\hat{L}(\hat{P}) h^{\prime}(\hat{L}(\hat{P}))-\text { h.c. }\right)\right) \sim \hat{A}\left(\exp \left(t \hat{H}_{h}\right)(\hat{P})\right) \\
& \sim A\left(\exp \left(t H_{h}\right)(P)\right)
\end{aligned}
$$

(The second similarity is readily verified on $\hat{\Omega}_{r}^{c}$ by using (2.61) and (5.55)-(5.57).)

Applying this theorem to the equilibria (5.69) for the $H_{k}$ flow, we obtain once again the isospectrality relation (5.42).

The partition function for $H_{k}$ diverges on $\Omega^{\# c}$ due to the infinite range of variation of $\delta_{s}$ (on which $H_{k}$ does not depend). On the compact quotient manifold $\Omega^{\#}$ we do get finite partition functions, of course. Since $\Omega^{\#} \backslash \Omega$ has measure zero, the latter are given by (5.22), with (1.41) in force.

Theorem 5.10. One has

$$
Z_{k}(T)=\left(\frac{2 \pi}{|\mu|}\right)^{N} \int_{A_{N}^{b} \cap F_{N}(\beta)} \exp \left(-\sum_{j=1}^{N} \cos \left(|\beta| k \hat{p}_{j}\right) / T\right) d \hat{p}, \quad k=1,2, \ldots
$$

with $A_{N}^{b}$ given by (1.78) and $F_{N}(\mu)$ by (1.15). 
Proof. We may restrict the integration in (5.22) to $\Omega_{r}$, since $\Omega \backslash \Omega_{r}$ has measure zero. Then we can use the canonicity of the map $\Phi: \Omega_{r} \rightarrow \hat{\Omega}_{r}$ w.r.t. the coordinates $(x, p)$ on $\Omega_{r}$ and $(\hat{x}, \hat{p})$ on $\hat{\Omega}_{r}$. (The latter are defined by dualizing (1.41); this yields the integration region in (5.79), cf. also (1.35), (1.36) and (1.40).)

In the remainder of this section we restrict attention to the reduced phase space $M$ equipped with the renormalized symplectic form $\omega_{\text {ren }}$. By now, it will be plain how corresponding results for $\Omega^{\# c}=\mathbf{R}^{2} \times M$ and its quotient $\Omega^{\#}$ can be obtained. In contrast to the situation in Section 4.4 , it is notationally and conceptually more convenient to view $\phi$ as a symplectomorphism from $\left\langle\boldsymbol{M}, \omega_{\text {ren }}\right\rangle$ onto $\left\langle\hat{M},-\omega_{\text {ren }}\right\rangle$ with inverse $b$.

First, we study the limit $\tau \uparrow \pi / N$ at the level of dynamics. To this end we recall that we have

$$
\lim _{\tau \uparrow \pi / N} \exp \left(\beta \hat{\theta}_{J}\right)=\exp [i \pi(N+1-2 j) / N] \equiv x_{j}^{(0)}, j=1, \ldots, N
$$

on all of $\hat{M}$, cf. (5.54). Thus, for the entire function $h(x)=\sum_{k=0}^{\infty} a_{k} x^{k}$ we obtain from (5.53)

$$
\lim _{\tau \uparrow \pi / N} \hat{H}_{h}(\hat{z})=2 N \sum_{k \in N \mathbb{N}}(-)^{k(N-1)} \operatorname{Re} a_{k} \equiv r_{h}, \quad \forall \hat{z} \in \hat{M}
$$

We can get finite and non-constant limits, however, via an appropriate renormalization. Specifically, setting

$$
H_{h, r e n} \equiv\left(H_{h}-r_{h}\right) /(\pi-N \tau), \quad \hat{H}_{h, r e n} \equiv H_{h, r e n} \circ \mathrm{b}
$$

we obtain from (5.53) and (5.54)

$$
\begin{aligned}
\hat{H}_{h, 0}(\hat{z}) \equiv & \lim _{\tau \uparrow \pi / N} \hat{H}_{h, \text { ren }}(\hat{z}) \\
& =i \sum_{j=1}^{N}\left(x_{j}^{(0)} h^{\prime}\left(x_{j}^{(0)}\right)-\text { c.c. }\right) \cdot \\
& \left(-\frac{(N+1-2 j)}{N}+\frac{2}{(\hat{z}, \hat{z})}\left(\sum_{k=j}^{n}\left|\hat{z}_{k}\right|^{2}-\frac{1}{N} \sum_{k=1}^{N} k\left|\hat{z}_{k}\right|^{2}\right)\right)
\end{aligned}
$$

Introducing

$$
\begin{gathered}
v_{h, j, 0} \equiv 2 i\left(x_{j}^{(0)} h^{\prime}\left(x_{j}^{(0)}\right)-\text { c.c. }\right), \quad j=1, \ldots, N \\
\omega_{h, l, 0} \equiv \sum_{J-1}^{l} v_{h, j, 0}-\frac{l}{N} \sum_{j=1}^{N} v_{h, j, 0}, \quad l=1, \ldots, n
\end{gathered}
$$


this can be rewritten

$$
\hat{H}_{h, 0}(\hat{z})=\left(\hat{z}, D_{h} \hat{z}\right) /(\hat{z}, \hat{z})
$$

where

$$
\begin{gathered}
D_{h} \equiv \operatorname{diag}\left(0, \omega_{h, 1,0}, \ldots, \omega_{h, n, 0}\right)+c_{h} \mathbb{1}_{N} \\
c_{h} \equiv-\frac{1}{2 N} \sum_{j=1}^{N}(N+1-2 j) v_{h, j, 0}
\end{gathered}
$$

The flow generated by the Hamiltonian $(5.86)$ on $\left\langle\hat{M},-\omega_{\text {ren }}\right\rangle$ is given by

$$
\exp \left(t \hat{H}_{h, 0}\right)(\hat{z})=\left(\hat{z}_{0}, \hat{z}_{1} \exp \left(i t \omega_{h, 1,0}\right), \ldots, \hat{z}_{n} \exp \left(i t \omega_{h, n, 0}\right)\right)
$$

cf. the paragraph containing (1.104). This agrees with the limit of the flow exp $\left(t \hat{H}_{h, r e n}\right)(\hat{z})$ for $\tau \uparrow \pi / N$, as should be the case, of course. (To calculate this limit, recall $\omega_{\text {ren }}$ differs by a factor $|\beta \mu| / 2(\pi-N \tau)$ from $\omega_{R}$, cf. (1.103), and use (5.55) -(5.57).) Introducing

$$
H_{h, 0} \equiv \hat{H}_{h, 0} \circ \mathrm{k} \circ \mathrm{f}_{0}
$$

and recalling $k$ and $f_{0}$ are the quotients of complex conjugation and Fourier transformation $F_{0}$ on $\mathbb{C}^{N}$ (cf. (4.128)), resp., we also conclude

$$
H_{h, 0}(z)=\left(z, A_{h} z\right) /(z, z), \quad A_{h} \equiv F_{0}^{*} D_{h} F_{0}
$$

For the special Hamiltonians (5.68) we have (using obvious notation)

$$
v_{k, j, 0}=-2 k \sin (\pi k(N+1-2 j) / N), \quad k=1,2, \ldots
$$

so we may as well restrict attention to $k<N$. Then we obtain for $N=2$

$$
\omega_{1,1,0}=-2, \quad c_{1}=1, \quad D_{1}=\operatorname{diag}(1,-1)
$$

and for $N>2$

$$
\begin{gathered}
\omega_{k, l, 0}=(-)^{k+1} \frac{k}{\sin (k \pi / N)}(\cos (2 k l \pi / N)-1), \quad k=1, \ldots, n \\
c_{k}=(-)^{k+1} \frac{k}{\sin (k \pi / N)}, \quad k=1, \ldots, n
\end{gathered}
$$




$$
\left(D_{k}\right)_{\nu \rho}=(-)^{k+1} \frac{k}{\sin (k \pi / N)} \delta_{\nu \rho} \cos (2 k \nu \pi / N), k=1, \ldots, n, \nu, \rho=0, \ldots, n
$$

Specializing to $k=1$, we obtain from (5.91)

$$
H_{1,0}(z)=\frac{\left(z, \Delta_{p e r} z\right)}{2(z, z)} \begin{cases}2 & (N=2) \\ \sin (\pi / N)^{-1} & (N>2)\end{cases}
$$

where $\Delta_{p e r}$ is the periodic lattice Laplacean

$$
\Delta_{p e r} \equiv\left(\begin{array}{ccccc}
0 & 1 & \cdots & 0 & 1 \\
1 & 0 & \cdots & 0 & 0 \\
\vdots & \vdots & \ddots & \vdots & \vdots \\
0 & 0 & \cdots & 0 & 1 \\
1 & 0 & \cdots & 1 & 0
\end{array}\right)
$$

It is straightforward to verify directly that the Hamiltonian (1.4), divided by $\pi-$ $N \tau$, yields the limit (5.97) upon taking $\tau \uparrow \pi / N$. (Recall (1.30), (1.98) to write exp $\left(\beta p_{j}\right) \rightarrow z_{j}\left|z_{j-1}\right| /\left|z_{j}\right| z_{j-1}$ (with $\left.z_{N} \equiv z_{0}\right)$, and use (5.77) to obtain the limits of $V_{j}^{b} /(\pi-N \tau)$.)

Finally, we study the question whether $M \simeq \mathbf{P}^{n}$ is a minimal completion of $M^{0}$ $\simeq \mathbf{C}^{* n}$ w.r.t. the non-complete flows on $M^{0}$. Just as for the cases $\hat{\mathrm{II}}_{\mathrm{nr}}$ and $\hat{\mathrm{II}}_{\text {rel }}$, this boils down to the question whether or not there exist points $P$ in the exceptional set $M^{e}=M \backslash M^{0}$ for which the orbit union

$$
\mathcal{O}(P) \equiv\left\{\exp \left(t H_{h}\right)(P) \mid t \in \mathbf{R}, h \in \mathscr{C}_{e}\right\}
$$

belongs to $M^{e}$. We begin with some (related) observations that are valid for any $\tau \in(0, \pi / N)$.

(i) The answer to the above question does not depend on whether or not one renormalizes the Hamiltonians via (5.82) and the symplectic form via (1.103). (Indeed, such renormalizations only give rise to a rescaling of the evolution parameter $t$.)

(ii) For any $P \in M$ we have

$$
\phi(\mathcal{O}(P))=\{\hat{z} \in \hat{M} \mid \hat{\delta}(\hat{z})=\hat{\delta}(\phi(P))\}
$$

Thus $\mathcal{O}(P)$ either equals a point (viz., iff $P=\varepsilon_{0}, \ldots, \varepsilon_{n}$ ) or a torus $\mathbf{T}^{j(P)}, j(P) \in\{1$, $\ldots, n\}$. (Fixing $\hat{\boldsymbol{P}} \in \hat{M}$ and letting $h$ vary over $\mathscr{C}_{e}$, the vector $\left(v_{h, 1}, \ldots, v_{h, N}\right)(\hat{\boldsymbol{P}})$ varies over all of $\mathbf{R}^{N}$, cf. $(5.57)$; but then the vector $\left(\omega_{h, 1}, \ldots, \omega_{h, n}\right)(\hat{\boldsymbol{P}})$ varies over all of $\mathbf{R}^{n}$, since the connecting matrix has rank $n$, cf. (5.56); hence the assertion follows from the reduced version of (5.55).) 
(iii) The 'superfluous' set

$$
M_{s f} \equiv\left\{P \in M^{e} \mid \mathcal{O}(P) \subset M^{e}\right\}
$$

is closed. (If $P_{0} \in M^{e}, t \in \mathbf{R}^{*}$ and $h \in \mathscr{C}_{e}$ are such that $\exp \left(t H_{h}\right)\left(P_{0}\right) \notin M^{e}$, then one has $\exp \left(t H_{h}\right)(P) \notin M^{e}$ for $P$ near $P_{0}$.)

(iv) One has

$$
e_{\nu} \not \subset M_{s f}, \quad \nu=0, \ldots, n
$$

Thus one can find open neighborhoods of $e_{0}, \ldots, e_{n}$ not belonging to $M_{s f}$. (The point $\mathrm{p}_{0} \in M^{0}$ is a fixed point of $\phi$, cf. (4.115)-(4.118); from (5.100) one then infers that $\mathcal{O}\left(\mathrm{p}_{0}\right)$ is $n$-dimensional and contains $e_{0}, \ldots, e_{n}$.)

(v) One has

$$
M_{s f}=\emptyset \Longleftrightarrow \hat{\delta}\left(\phi\left(M^{0}\right)\right)=\left(a_{n}^{b}\right)^{c l}
$$

(This equivalence readily follows by combining (5.100) and (5.101).)

Next, we show that $M$ is a minimal completion of $M^{0}$ for $N=2,3$ :

$$
M_{s f}=\emptyset, \quad \forall \tau \in(0, \pi / N), \quad N=2,3
$$

For $N=2$ this is obvious : the set $M^{e}=\{c, d\}$ in Figure 3 lies on the $H_{1}$-orbit (great circle) through $p$ and $q$. (Equivalently, one need only specialize (5.102) to $N=2$.) For $N=3$ this can be seen as follows (cf. Figure 4). Assume $M_{s f}$ is non-empty and fix $P \in M_{s f}$. Now $\mathcal{O}(P)$ is connected and $e_{0}, e_{1}, e_{2} \notin M_{s f}$, so $\mathcal{O}(P)$ lies above one of the 3 simplex edges. Since $\mathbb{T}^{2}$ does not embed in $\mathbb{C}^{*}$, the orbit union $\mathcal{O}(P)$ is either a circle or a point. In the latter case it would follow that $\mathcal{O}(P)$ equals one of the equilibria $\varepsilon_{0}, \varepsilon_{1}, \varepsilon_{2}$ lying above the barycenter, a contradiction. Thus $\mathcal{O}(P)$ is a circle. But then we have $\phi(\mathcal{O}(P)) \subset \hat{M}^{e}$, cf. (5.100). Using the notation (4.156) and the corresponding identification, we deduce $\mathcal{O}(P) \subset V_{b}^{e}$. But $V_{b}^{e}$ consists of 9 points, cf. (4.158), so we arrive again at a contradiction. Hence, (5.104) is now proved.

We now proceed to our last result, which says in particular $M_{s f}=\emptyset$ for $\tau$ near $\pi / N$. To this end we first introduce the orbit union and superfluous set for the limiting $\tau$-value $\pi / N$ by putting

$$
\begin{gathered}
\mathcal{O}_{0}(P) \equiv\left\{\exp \left(t H_{h, 0}\right)(P) \mid t \in \mathbf{R}, h \in \mathscr{C}_{e}\right\} \\
M_{s f, 0} \equiv\left\{P \in M^{e} \mid \mathcal{O}_{0}(P) \subset M^{e}\right\}
\end{gathered}
$$

It is easy to rephrase the observations (ii) $-(v)$ for $\tau=\pi / N$ by using renormalized 
quantities

$$
\hat{\delta}_{\nu, 0} \equiv\left|\hat{z}_{\nu}\right|^{2} /(\hat{z}, \hat{z}), \quad \nu=0, \ldots, n
$$

so we shall not spell this out. (Note that $\left(\hat{\delta}_{1,0}, \ldots, \hat{\delta}_{n, 0}\right)$ varies over the closure of the Weyl alcove (1.8) with $|\mu|=\pi$, cf. also (1.97).)

Theorem 5.11. One has

$$
M_{s f, 0}=\emptyset, \quad \forall N \geq 2
$$

There exists $\varepsilon(N)>0$ such that

$$
M_{s f}=\emptyset, \quad \forall \tau \in(\pi / N-\varepsilon(N), \pi / N)
$$

and $\varepsilon(N)$ may be taken equal to $\pi / N$ for $N=2,3$.

Proof. Assume $M_{s f, 0}$ is not empty and fix $P \in M_{s f, 0}$. Choosing a vector $\phi \in$ $\mathbf{C}^{N} \backslash\{0\}$ that descends on $P$ under the projection $\mathbf{C}^{N} \backslash\{0\} \rightarrow \mathbf{P}^{n}$, the assumption entails that when we fix $t \in \mathbf{R}$ and $h \in \mathscr{C}_{e}$, at least one of the coordinates of the vector $\exp \left(-i t A_{h}\right) \phi$ vanishes. (Recall the latter vector descends on $\exp \left(t H_{h, 0}\right)(P)$.) Letting $t$ vary, it easily follows that at least one of the coordinates vanishes for all $t \in \mathbf{R}$. That is, for any $h \in \mathscr{C}_{e}$ we can find $\rho \in\{0, \ldots, n\}$ such that

$$
\left(e_{\rho}, \exp \left(-i t A_{h}\right) \psi\right)=0, \quad \forall t \in \mathbf{R}
$$

Equivalently, using (5.91) we have

$$
\sum_{j=1}^{N} \omega^{-\rho(j-1)} \exp \left(-i t D_{h, j j}\right)\left(\boldsymbol{F}_{0} \phi\right)_{j}=0, \quad \forall t \in \mathbf{R}
$$

But if we now choose $h$ such that the numbers $D_{h, 11}, \ldots, D_{h, N N}$ are distinct, then we infer from (5.111) that $\phi=0$, a contradiction. Thus the first assertion is proved.

Next, assume there exists a sequence $\tau_{k} \uparrow \pi / N, k \rightarrow \infty$, such that $M_{s f}\left(\tau_{k}\right)$ is non-empty. Choosing $\boldsymbol{P}_{k} \in M_{s f}\left(\tau_{k}\right) \subset \boldsymbol{M}^{e}$, we can find a subsequence $\boldsymbol{P}_{i_{k}}$ with limit $P_{0} \in M^{e}$, as $M^{e}$ is compact. But then we may deduce $\mathcal{O}_{0}\left(P_{0}\right) \subset M^{e}$ from $\mathcal{O}\left(P_{i_{k}}\right) \subset$ $M^{e}$, since $\phi$ converges uniformly to $\mathrm{k}^{\circ} \circ \mathrm{f}_{0}$, cf. Theorem 4.9. This contradicts (5.108), so (5.109) results. The last assertion has already been proved, cf. (5.104).

We conjecture that in (5.109) one may take $\varepsilon(N)=\pi / N$ for $N=4,5, \ldots$, too. 


\section{Appendix A. Some Algebraic Lemmas}

In this paper we will make use of the Cauchy matrix from II, taking $q, p \rightarrow a$ and $M \rightarrow N$. Thus we have

$$
C(\beta, \mu, g ; a)_{j k} \equiv e^{-\beta a_{j} / 2} \frac{\operatorname{sh}\left(\frac{i}{2} \beta \mu g\right)}{\operatorname{sh} \frac{\beta}{2}\left(a_{j}-a_{k}+i \mu g\right)} e^{-\beta a_{k} / 2}, j, k=1, \ldots, N
$$

and Cauchy's identity reads

$$
|C|=e^{-\beta \Sigma_{j} a_{j}} \prod_{j<k} \frac{\operatorname{sh}^{2} \frac{\beta}{2}\left(a_{j}-a_{k}\right)}{\operatorname{sh} \frac{\beta}{2}\left(a_{j}-a_{k}+i \mu g\right) \operatorname{sh} \frac{\beta}{2}\left(a_{j}-a_{k}-i \mu g\right)}
$$

cf. II, Appendix B. The Lax matrix arising for the $\mathrm{II}_{\text {rel }}$ system (and for the $\mathrm{I}_{\text {rel }}$ system studied in I ) can be seen to be positive by using the following lemma.

Lemma A.1. Let $\beta, g \in \mathbf{R}^{*}$ and $\mu \in i \mathbf{R}$. For any $q \in W_{N}$ (where $W_{N}$ is defined by (1.9)) the matrix

$$
C_{j k}^{\prime} \equiv e^{\mu q_{j} / 2} C(\mu, \beta, g ; q)_{j k} e^{\mu q_{k} / 2}
$$

is positive.

Proof. The restrictions ensure that $C^{\prime}$ is well defined and self-adjoint. Moreover, $C^{\prime}$ has positive principal minors in view of (A.2).

For the systems dual to the $\mathbb{I I I}_{\mathrm{nr}}$ and $\mathrm{III}_{\text {rel }}$ systems, and for the $\mathrm{III}_{\mathrm{b}}$ system (which is self-dual) it is expedient to employ a different Cauchy matrix. This matrix (denoted $\tilde{C}$ ) involves the quantities

$$
\begin{aligned}
& l_{j}(\beta, \mu, g ; a) \equiv e^{\beta a_{j}} \prod_{k \neq j} \frac{\operatorname{sh} \frac{\beta}{2}\left(a_{j}-a_{k}+i \mu g\right)}{\operatorname{sh} \frac{\beta}{2}\left(a_{j}-a_{k}\right)}, \\
& r_{j}(\beta, \mu, g ; a) \equiv l_{j}(\beta, \mu,-g ; a)
\end{aligned}
$$

and its pivotal orthogonality property hinges on the following lemma. 
Lemma A.2. Let $(\beta, \mu, g, a) \in \mathbf{C}^{3+N}$ be such that $C_{j k}, l_{j}, r_{k}, j, k=1, \ldots, N$, are well defined and $|C| \neq 0$. Then one has

$$
\left(C^{-1 t}\right)_{j k}=l_{j} C_{j k} r_{k}
$$

Proof. This identity can be verified by a straightforward calculation using (A.2) ; cf. Lemma B2 in II for a slightly more general result.

Defining now

$$
\widetilde{C}_{j k} \equiv l_{j}^{1 / 2} C_{j k} r_{k}^{1 / 2}
$$

it follows from the lemma that $\widetilde{C} \in O(N, \mathrm{C})$. Since diagonal sign matrices are orthogonal, this property holds true irrespective of the sign conventions for the square roots.

In Chapter 2, however, we will wind up with positive quotients in the product occurring in (A.4), and correspondingly we may and will take positive square roots throughout. To be specific, the construction of the action-angle map in Chapter 2 leads to the inequality

$$
\prod_{l \neq j} \frac{\operatorname{sh} \frac{\beta}{2}\left(a_{j}-a_{l}+d\right)}{\operatorname{sh} \frac{\beta}{2}\left(a_{j}-a_{l}\right)} \prod_{m \neq k} \frac{\operatorname{sh} \frac{\beta}{2}\left(a_{k}-a_{m}-d\right)}{\operatorname{sh} \frac{\beta}{2}\left(a_{k}-a_{m}\right)}>0, j, k=1, \ldots, N, d \equiv|\mu g|
$$

Here, one has in addition

$$
\begin{gathered}
a_{N}<\cdots<a_{1}, \quad \beta=0 \\
a_{N}<\cdots<a_{1}, \quad \beta>0 \\
-\pi /|\beta|<a_{N}<\cdots<a_{1} \leq \pi /|\beta|, \quad-i \beta>0, \quad d<2 \pi / N|\beta|
\end{gathered}
$$

for the cases $\mathrm{III}_{\mathrm{nr}}, \mathrm{III}_{\mathrm{rel}}, \mathrm{II}_{\mathrm{b}}$, resp. A cornerstone in the construction is then that this inequality entails a further restriction on the actions $a_{1}, \ldots, a_{N}$ (implying in particular positivity of the quotients in (A.4)).

We shall detail and prove this restriction in the next lemma. To this end we introduce

$$
\begin{aligned}
A_{N} & \equiv\left\{a \in \mathbf{R}^{N} \mid d_{j}>d, \quad j=1, \ldots, N-1\right\}, d_{j} \equiv a_{j}-a_{j+1} \\
\hat{F}_{N}^{b} & \equiv\left\{a \in \mathbf{R}^{N}\left|d_{j}>d, \quad j=1, \ldots, N,-\pi /\right| \beta\left|<a_{N}, a_{1} \leq \pi /\right| \beta \mid\right\}, d_{N} \\
& \equiv a_{N}-a_{1}+2 \pi /|\beta|
\end{aligned}
$$


Note the definition (A.11) agrees with (1.49), whereas $\hat{F}_{N}^{b}$ is a fundamental set for a $\mathbf{Z}$-action on the set $A_{N}^{b}$ defined by (1.78) (cf. the paragraph containing (2.97)).

Lemma A.3. Suppose (A.7) holds true. If, in addition, (A.8), (A.9) or (A.10) are valid, then one has $a \in A_{N}, A_{N}$ or $\hat{F}_{N}^{b}$, resp.

Proof. Fixing $k \in\{1, \ldots, N-1\}$, let us assume $d_{k}=d$. Then the factor with $m$ $=k+1$ at the lhs of (A.7) vanishes, contradicting (A.7). Thus one must have either $d_{k} \in(0, d)$ or $d_{k}>d$. Let us now first start from (A.8) or (A.9). We assume that at least one $d_{k}$ is smaller than $d$ and derive a contradiction to (A.7). We distinguish three cases.

(i) One has

$$
d_{k}<d, k=1, \ldots, N-1
$$

Taking $j=1$ and $k=N-1$ in (A.7), it easily follows that all factors but the one for which $m=N$ are positive, contradicting (A.7). This takes care of the special case $N=2$, so we may now assume $N>2$.

(ii) There exists $k_{0} \in\{1, \ldots, N-2\}$ such that

$$
d_{k}<d, k=1, \ldots, k_{0}, d_{k_{0}+1}>d
$$

Taking $j=k_{0}+2, k=k_{0}$, we then obtain positive factors at the lhs of (A.7), but for the factor with $m=k_{0}+1$, a contradiction.

(iii) There exists $k_{0} \in\{1, \ldots, N-2\}$ such that

$$
d_{k}>d, k=1, \ldots, k_{0}, d_{k_{0}+1}<d
$$

Taking once more $j=k_{0}+2, k=k_{0}$ in (A.7) we now obtain positive factors but for the factor with $l=k_{0}+1$. This contradiction completes the proof of the lemma when (A.8) or (A.9) is assumed to hold.

Finally, we start from the hypothesis (A.10). First, we assume that there exists at least one $k \in\{1, \ldots, N-1\}$ such that $d_{k}<d$. Then the above case distinction and the corresponding conclusions apply verbatim. As this is not immediate, we add two exemplary verifications. In the first case one infers positivity of $\sin \frac{|\beta|}{2}\left(a_{1}-a_{N}+d\right) / \sin \frac{|\beta|}{2}\left(a_{1}-a_{N}\right)$ by noting

$$
d_{k}<d, k=1, \ldots, N-1 \Longrightarrow a_{1}-a_{N}<(N-1) d<2 \pi /|\beta|-d
$$

and in the second case (taking $\left.k_{0}<N-2\right)$ one gets positivity of $\sin \frac{|\beta|}{2}\left(a_{k_{0}+2}-\right.$ $\left.a_{N}+d\right) / \sin \frac{|\beta|}{2}\left(a_{k_{0}+2}-a_{N}\right)$ by noting 


$$
a_{k_{0}+2}-a_{N}=a_{k_{0}+1}-a_{N}-d_{k_{0}+1}<a_{1}-a_{N}-d<2 \pi /|\beta|-d
$$

As a consequence, (A.10) implies $d_{k}>d, k=1, \ldots, N-1$. It remains to prove $d_{N}>d$. But this becomes clear when one takes $j, k=1$ in (A.7) : assuming $d_{N} \leq$ $d$, the factor with $l=N$ is not positive, whereas all other factors are (note $a_{1}-a_{N-1}$ $\left.=\left(a_{1}-a_{N}\right)+\left(a_{N}-a_{N-1}\right)<2 \pi /|\beta|-d\right)$.

The next lemma will be used in Section 2.3 to show that in certain phase space points the spectrum of the Lax matrix consists of the $N$ th roots of a phase factor.

Lemma A.4. For any function $G:(0,2] \rightarrow \mathrm{C}$ and any $l \in\{1, \ldots, N-1\}$ one has

$$
\sum_{\substack{I \subset\{1, \ldots, N\} \\|I|=l}} \prod_{\substack{j \in I \\ k \notin I}} \omega^{j} G\left(\left|\omega^{j}-\omega^{k}\right|\right)=0, \quad \omega \equiv e^{2 \pi i / N}
$$

Proof. We rearrange the sum such that subsets $I$ related by cyclic permutations of $\{1, \ldots, N\}$ are grouped together. For any such group the factor $\Pi G(\cdots)$ takes the same value. Therefore, fixing $I_{0}$ with $\left|I_{0}\right|=l$, we need only show

$$
\sum_{\substack{|I|=I \\ I \sim I}} \prod_{J \in I} \omega^{j}=0
$$

where $\sim$ denotes cyclic equivalence.

First, consider the case where all cyclic translates of $I_{0}$ are distinct. Then the lhs of (A.17) reads

$$
\sum_{m=0}^{N-1} \omega^{l m} \prod_{j \in I} \omega^{j}
$$

Since $l \in\{1, \ldots, N-1\}$, this vanishes, as asserted. Next, assume that after $M<N$ cyclic translations of $I_{0}$ one reobtains $I_{0}$. This entails

$$
\prod_{\jmath \in I} \omega^{j}=\omega^{l M} \prod_{J \in I} \omega^{j}
$$

so that one must have $l M=n N, n \in\{1, \ldots, M-1\}$. But then the lhs of (A.17) can be rewritten

$$
\sum_{m=0}^{M-1} \omega^{l m} \prod_{\jmath \in I} \omega^{j}=\left(\prod_{\jmath \in I} \omega^{j}\right)_{m=0}^{M-1} e^{2 \pi i n m / M}
$$

which vanishes, too.

We proceed with Lemma A.5, whose corollary Lemma A.6 will be used in Chapter 4 to find the points in phase space where the Lax matrix has minimal 
spectral gaps. (These points are the equilibrium points, cf. Chapter 5.) Lemma A.5 can also be exploited to find the ground state and ground state energies of the quantized systems. Finally, a corollary of the proof will be quite useful in Sections 2.1 and 2.3 .

Lemma A.5. Let $y \in \mathbb{C}^{N}, z \in \mathbb{C}$ be such that the matrix

$$
E_{j k} \equiv c_{j} \frac{\operatorname{sh} z}{\operatorname{sh}\left(y_{j}-y_{k}+z\right)}, j, k=1, \ldots, N
$$

with

$$
c_{j} \equiv \prod_{l \neq j} \frac{\operatorname{sh}\left(y_{j}-y_{l}+z\right)}{\operatorname{sh}\left(y_{j}-y_{l}\right)}, j=1, \ldots, N
$$

is well defined. Then the roots of the polynomial $\lambda \longmapsto\left|E-\lambda \mathbb{1}_{N}\right|$ are given by

$$
\lambda_{j}=e^{(N+1-2 j) z}, \quad j=1, \ldots, N
$$

Proof. Due to Cauchy's identity (A.2) the symmetric functions of $E$ are given by

$$
S_{k}=\sum_{|I|=k} \prod_{\substack{l \in I \\ J \notin I}} \frac{\operatorname{sh}\left(y_{i}-y_{j}+z\right)}{\operatorname{sh}\left(y_{i}-y_{j}\right)}
$$

We claim that $S_{k}$ does not depend on $y$. Taking this for granted, we set $y_{k} \equiv-k \Lambda$, $\Lambda>0$, in $E$ to obtain

$$
\lim _{\Lambda \rightarrow \infty} E=\operatorname{diag}\left(e^{(N-1) z}, e^{(N-3) z}, \ldots, e^{(-N+1) z}\right)
$$

and the lemma follows. Thus it remains to prove the claim.

First, we note that $S_{k}$ is symmetric in $y_{1}, \ldots, y_{N}$, so we need only show constancy in $y_{1}$. To this end we fix $y_{2}, \ldots, y_{N}, z$ in general position, so that each of the terms at the rhs has at most simple poles for $y_{1}=y_{l}(\bmod 2 \pi i), l>1$. Clearly, $S_{k}$ is $2 \pi i$-periodic in $y_{1}$ and converges to a constant for $\operatorname{Re} y_{1} \rightarrow \pm \infty$. Therefore, by Liouville's theorem it suffices to prove that the residues vanish. Moreover, by symmetry and periodicity we need only consider the pole at $y_{1}=y_{2}$.

To show that the residue at this pole vanishes, we first observe that when the index set $I$ does not contain the indices 1 and 2 or contains both of them, then the corresponding term in $S_{k}$ has no pole at $y_{1}=y_{2}$. Next, we pair off the remaining $I$ by setting $I_{1}=\{1\} \cup J, I_{2}=\{2\} \cup J$, where $1,2 \not \subset J$. Then the residue sum for any such pair vanishes. Indeed, omitting the singular factors $1 / \operatorname{sh}\left(y_{1}-y_{2}\right)$ and $1 / \operatorname{sh}\left(y_{2}-y_{1}\right)$ in $I_{1}$ and $I_{2}$, resp., and setting $y_{1}=y_{2}$ in the remaining products, the 
latter are manifestly equal.

The application of this lemma to spectral properties of the Lax matrix arises upon setting

$$
y_{j}=\frac{i \pi}{2 N}(N+1-2 j), \quad j=1, \ldots, N
$$

Then one readily verifies

$$
c_{j}=\prod_{l \neq j} \frac{\sin \left(\frac{\pi}{N}(j-l)+i z\right)}{\sin \frac{\pi}{N}(j-l)}=r(i z)
$$

where

$$
r(\tau) \equiv \frac{\sin \left(\tau+\frac{\pi}{N}\right)}{\sin \frac{\pi}{N}} \frac{\sin \left(\tau+\frac{2 \pi}{N}\right)}{\sin \frac{2 \pi}{N}} \cdots \frac{\sin \left(\tau+\frac{(N-1) \pi}{N}\right)}{\sin \frac{(N-1) \pi}{N}}=\frac{\sin N \tau}{N \sin \tau}
$$

Hence, setting $z \equiv i \tau$, we may rewrite $E$ as

$$
E_{j k}=\frac{\sin N \tau}{N \sin \left(\tau-\frac{\pi}{N}(j-k)\right)}
$$

Then

$$
E_{1} \equiv \lim _{\tau \rightarrow 0} \frac{1}{\tau}\left(E-\mathbf{1}_{N}\right)
$$

reads

$$
\left(E_{1}\right)_{j k}=\left(\delta_{j k}-1\right) \frac{1}{\sin \frac{\pi}{N}(j-k)}
$$

Lemma A.6. Let $\tau \in \pm(0, \pi / N)$ or $i \tau \in \mathbf{R}^{*}$, and let $E$ and $E_{1}$ be given by (A.29) and (A.30), resp. Then $E$ and $E_{1}$ have simple spectrum given by

$$
\begin{gathered}
\sigma(E)=\left\{e^{(N-1) i \tau}, e^{(N-3) i \tau}, \ldots, e^{(-N+1) i \tau}\right\} \\
\sigma\left(E_{1}\right)=i\{N-1, N-3, \ldots,-N+1\}
\end{gathered}
$$


Proof. The assertion about $E$ follows from Lemma A.5. To prove (A.33) we rewrite $E$ as

$E=\frac{\sin N \tau}{N}\left(\frac{1_{N}}{\sin \tau}+\frac{S}{\sin \left(\tau+\frac{\pi}{N}\right)}+\frac{S^{2}}{\sin \left(\tau+\frac{2 \pi}{N}\right)}+\cdots+\frac{S^{N-1}}{\sin \left(\tau+\frac{(N-1) \pi}{N}\right)}\right)$

(A.34)

where $S$ is the antiperiodic shift,

$$
S \equiv\left(\begin{array}{cccc}
0 & 1 & \cdots & 0 \\
\vdots & \vdots & \ddots & \vdots \\
0 & 0 & \cdots & 1 \\
-1 & 0 & \cdots & 0
\end{array}\right)
$$

Now $S$ has eigenvalues

$$
s_{k}=e^{i \pi(2 k-1) / N}, \quad k=1, \ldots, N
$$

and corresponding eigenvectors

$$
u_{j}^{(k)}=N^{-1 / 2} e^{i \pi(2 j k-j-k) / N}, \quad k, j=1, \ldots, N
$$

as is easily verified (and well known). Thus $u^{(1)}, \ldots, u^{(N)}$ is an eigenvector basis for $E$, and so (A.33) follows from (A.30) and (A.32).

As a corollary we obtain the remarkable identities

$$
\sin N \tau \sum_{j=0}^{N-1} \frac{e^{i \pi j(2 k-1) / N}}{\sin \left(\tau+\frac{j \pi}{N}\right)}=N e^{i \tau(N+1-2 k)}, \quad k=1, \ldots, N
$$

Indeed, these follow upon combining (A.32), (A.29) and (A.34)-(A.36). (To verify that the $1-1$ correspondence works out right, one need only check (A.38) for $\tau \downarrow-\pi / N_{\text {.) }}$

Our last lemma amounts to yet another functional identity, viz.,

$$
\operatorname{sh} z \sum_{k=1}^{N} \frac{e^{y_{J}-y_{k}}}{\operatorname{sh}\left(y_{k}-y_{j}+z\right)} \prod_{l \neq k} \frac{\operatorname{sh}\left(y_{k}-y_{l}+z\right)}{\operatorname{sh}\left(y_{k}-y_{l}\right)}=e^{(1-N) z}, j=1, \ldots, N
$$

It will be used in the proof of Lemma 2.3. 
Lemma A.7. One has

$$
C r=e^{(1-N) z} \zeta, \quad \zeta \equiv(1,1, \ldots, 1)
$$

where $C$ and $r$ are given by (A.1) and (A.4), resp.

Proof. Putting $y_{j}=-\beta a_{j} / 2, z=i \beta \mu g / 2$ in (A.1), (A.4) one sees that (A.40) is equivalent to (A.39). To prove (A.39), we note that the lhs is $2 \pi i$-periodic in $y_{j}$ and bounded for $\left|\operatorname{Re} y_{j}\right| \rightarrow \infty$. The residue sums at the (generically) simple poles $y_{j}=y_{l}(\bmod 2 \pi i)$ are easily checked to vanish, so that the lhs does not depend on $y_{j}$ by virtue of Liouville's theorem. Thus it is equal to its limit for $\operatorname{Re} y_{j} \rightarrow-\infty$, which yields (A.39).

\section{References}

[1] Ruijsenaars, S. N. M., Action-angle maps and scattering theory for some finite-dimensional integrable systems. I. The pure soliton case, Comm. Math. Phys., 115 (1988), 127-165.

[2] Action-angle maps and scattering theory for some finite-dimensional integrable systems. II. Solitions, antisolitons, and their bound states, Publ. RIMS, Kyoto Univ., 30 (1994), 865-1008.

[ 3 ] Olshanetsky, M. A. and Perelomov, A. M., Classical integrable finite-dimensional systems related to Lie algebras, Phys. Reps., 71 (1981), 313-400.

[4] Ruijsenaars, S. N. M. and Schneider, H., A new class of integrable systems and its relation to solitons, Ann. Phys. (NY), 170 (1986), 370-405.

[5] Ruijsenaars, S. N. M., Complete integrability of relativistic Calogero-Moser systems and elliptic function identities, Comm. Math. Phys., 110 (1987), 191-213.

[6] - Finite-dimensional soliton systems, Integrable and superintegrable systems, Kupershmidt, B. (ed.), Singapore, World Scientific, (1990), 165-206.

[ 7 ] A positive energy dynamics and scattering theory for directly interacting relativistic particles, Ann. Phys. (NY), 126 (1980), 399-449.

[8] Kazhdan, D., Kostant, B. and Sternberg, S., Hamiltonian group actions and dynamical systems of Calogero type, Comm. Pure Appl. Math., 31 (1978), 481-507.

[9] Adler, M., Completely integrable systems and symplectic actions, J. Math. Phys., 20 (1979), 6067.

[10] Airault, H., McKean, H. P. and Moser, J., Rational and elliptic solutions of the Korteweg-de Vries equation and a related many-body problem, Comm. Pure Appl. Math., 30 (1977), 95-148.

[11] Tuynman, G. M. and Wiegerinck, W. A. J. J., Central extensions and physics, J. Geom. Phys., 4 (1987), 207-258. 
